\title{
Modelo fatorial com cargas funcionais para séries temporais
}

\author{
Duván Humberto Cataño Salazar
}

\author{
TESE APRESENTADA \\ $\mathrm{AO}$ \\ INSTITUTO DE MATEMÁTICA E EsTATÍSTICA \\ DA \\ UNIVERSIDADE DE SÃo PAUlO \\ PARA \\ OBTENÇÃO DO TÍTULO \\ $\mathrm{DE}$ \\ DOUTOR EM CIÊNCIAS
}

\author{
Programa: Estatística \\ Orientadora: Profa. Dra. Chang Chiann
}

Coorientador: Prof. Dr. Daniel Peña Sánchez de Rivera

Durante o desenvolvimento deste trabalho o autor recebeu auxílio financeiro da CAPES/CNPq 


\section{Modelo fatorial com cargas funcionais para séries temporais}

Esta versão da tese contêm as correções e alterações sugeridas pela Comissão Julgadora durante a defesa da versão original do trabalho, realizada em 12/03/2018. Uma cópia da versão original está disponível no Instituto de Matemática e Estatística da Universidade de São Paulo.

Comissão Julgadora:

- Prof. Dra. Chang Chiann (orientadora) - IME-USP

- Prof. Dr. Pedro Alberto Morettin - IME-USP

- Prof. Dr. Aluísio de Souza Pinheiro - IMECC - UNICAMP

- Prof. Dra. Thelma Sáfadi - DES - UFLA

- Prof. Dr. Carlos Vladimir Rodríguez - UC3M 


\section{Agradecimentos}

Minha família, Humberto, Nelly, Luz Dary e o amor da minha vida a Paula. Agradeço a minha orientadora Chang Chiann por seu importante e incondicional apoio. Aos professores Pedro Morettin, Thelma Sáfadi, Aluísio de Souza e Vladimir Rodríguez pelos comentários e sugestões durante o processo de revisão.

Ao professor Daniel Peña por seus aportes e ajuda durante o estágio na UC3M.

Aos amigos que fiz, pelos agradáveis momentos, diversão e discussão, dos quais destaco: Lina, Hugo, Marcia, Andrea, Mario, Roger, Edwin, Roldão, Víctor, Fernando, Kim, Eliza Graciano e Eliza González. Aos amigos do futebol, pelos bons momentos: Cristian, Saulo, Clodo, Magrão, Calixto, Morán, Germancho, Marcelo, Fabio e Sergio.

Aos funcionários do IME, especialmente Regiane, Cecília e Sandra.

Ao CNPq e à CAPES, pelo apoio financeiro.

A todos os que deixei de mencionar, por esquecer no momento, e que também contribuíram no desenvolvimento deste trabalho, muito obrigado.

Obrigado IME - USP - São Paulo - Brasil! 


\title{
Resumo
}

\section{Modelo fatorial com cargas funcionais para séries temporais}

\begin{abstract}
No contexto dos modelos fatoriais existem diferentes metodologias para abordar a modelagem de séries temporais multivariadas que exibem uma estrutura não estacionária de segunda ordem, co- movimentos e transições no tempo. Modelos com mudanças estruturais abruptas e restrições rigorosas (muitas vezes irreais) nas cargas fatoriais, quando elas são funções determinísticas no tempo, foram propostos na literatura para lidar com séries multivariadas que possuem essas características. Neste trabalho, apresentamos um modelo fatorial com cargas variando continuamente no tempo para modelar séries temporais não estacionárias e um procedimento para sua estimação que consiste em dois estágios. No primeiro, os fatores latentes são estimados empregando os componentes principais das séries observadas. Em um segundo estágio, tratamos estes componentes principais como co-variáveis e as cargas funcionais são estimadas através de funções de ondaletas e mínimos quadrados generalizados. Propriedades assintóticas dos estimadores de componentes principais e de mínimos quadrados dos coeficientes de ondaletas são apresentados. O desempenho da metodologia é ilustrado através de estudos de simulação. Uma aplicação do modelo proposto no mercado spot de energia do Nord Pool é apresentado.
\end{abstract}

Palavras-chave: Modelos Fatoriais Aproximados, Estacionaridade Local, Componentes Principais, Ondaletas, Mínimos Quadrados Generalizados. 


\section{Abstract}

\section{Factor model with functional loadings}

\section{for time series}

In the context of the factor models there are different methodologies to modeling multivariate time series that exhibit a second order non-stationary structure, co-movements and transitions over time. Models with abrupt structural changes and strict restrictions (often unrealistic) in factor loadings, when they are deterministic functions of time, have been proposed in the literature to deal with multivariate series that have these characteristics. In this work, we present a factor model with time-varying loadings continuously to modeling non-stationary time series and a procedure for its estimation that consists of two stages. First, latent factors are estimated using the principal components of the observed series. Second, we treat principal components obtained in first stage as covariate and the functional loadings are estimated by wavelet functions and generalized least squares. Asymptotic properties of the principal components estimators and least squares estimators of the wavelet coefficients are presented. The performance of the methodology is illustrated by simulations. An application to the model proposed in the energy spot market of the Nord Pool is presented.

Keywords: Approximate Factor Models, Local Stationarity, Principal Components, Wavelets, Generalized Least Squares. 


\section{Sumário}

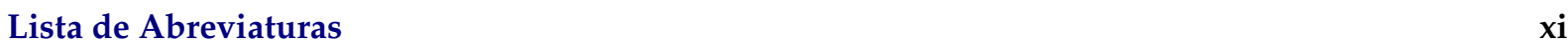

$\begin{array}{lc}\text { Lista de Notações } & \text { xiii }\end{array}$

1 Introdução $\quad 1$

2 Noções Básicas $\quad 5$

2.1 Processos Localmente Estacionários Multivariados . . . . . . . . . . . . . . . . 5

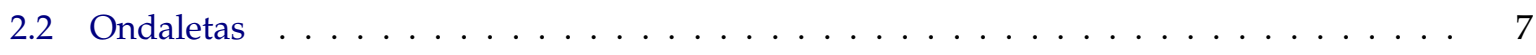

2.3 Modelos Fatoriais com Cargas Estáticas . . . . . . . . . . . . . . . . . . . 12

2.4 Modelo Fatorial com Cargas Variando no Tempo . . . . . . . . . . . . . . . . . . . . . 15

3 Modelo Fatorial com Cargas Funcionais $\quad 21$

3.1 O Modelo Proposto . . . . . . . . . . . . . . . . . . . . . . . . . . 21

3.1 Suposições . . . . . . . . . . . . . . . . . . . . 22

3.1.2 Estimador dos Componentes Principais . . . . . . . . . . . . . . . . 23

3.2 Identificabilidade . . . . . . . . . . . . . . . . . . . . . . . 24

3.3 Estimação das cargas funcionais . . . . . . . . . . . . . . . . . . . . 25

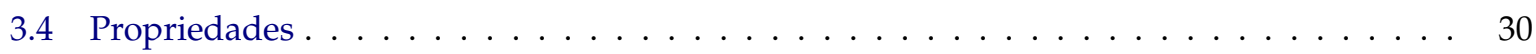

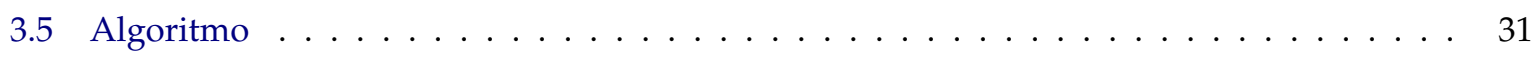

4 Simulações $\quad 33$

4.1 Esquema de simulação . . . . . . . . . . . . . . . . . . . . . . 33

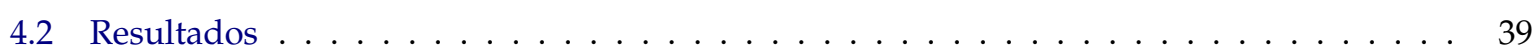

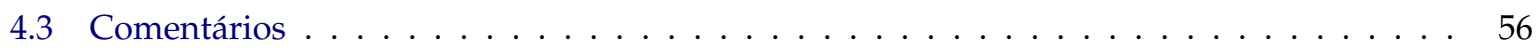

5 Aplicação $\quad 57$

5.1 Descrição dos dados . . . . . . . . . . . . . . . . . . . . 5 . . . . . .

5.2 Teste para não estacionariedade . . . . . . . . . . . . . . . . . . . . 59

5.3 Estimação . . . . . . . . . . . . . . . . . . . . . . . . . 61

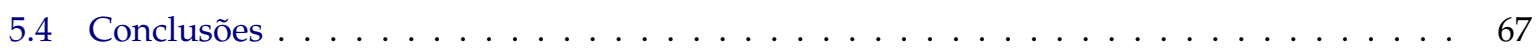


Referências Bibliográficas

$\begin{array}{lr}\text { A Apêndice } & 75\end{array}$

A.1 Definições e lemas auxiliares . . . . . . . . . . . . . . . . . . . . 75

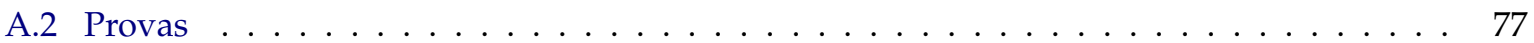




\section{Lista de Abreviaturas}

$\begin{array}{ll}\text { ACP } & \text { Análise de Componentes Principais } \\ \text { AMR } & \text { Análise Multirresolução } \\ \text { CP } & \text { Componentes Principais } \\ \text { DVAR } & \text { Autorregressivo Dinâmico Vetorial } \\ \text { EQM } & \text { Erro Quadrático Médio } \\ \text { MA( } \infty) & \text { Médias Móveis Infinita } \\ \text { M-GARCH } & \text { Heteroscedasticidade Condicional Autorregressiva Multivariada } \\ \text { MFLE } & \text { Modelo Fatorial Localmente Estacionário } \\ \text { MQ } & \text { Mínimos Quadrados } \\ \text { M-TAR } & \text { Threshold Autorregressivo Multivariado } \\ \text { MV } & \text { Máxima Verossimilhança } \\ \text { VAR } & \text { Autorregressivo Vetorial } \\ \text { VARMA } & \text { Autorregressivo e de Médias Móveis Vetorial }\end{array}$




\title{
Lista de Notações
}

\author{
$N \quad$ Número de séries temporais \\ T Número de observações em cada série temporal \\ $\mathbb{N} \quad$ Conjunto dos números naturais \\ $\mathbb{N}_{0} \quad \mathbb{N} \cup\{0\}$ \\ $\mathbb{Z} \quad$ Conjunto dos números inteiros \\ $\mathbb{R} \quad$ Conjunto dos números reais \\ $\mathbb{C} \quad$ Conjunto dos números complexos \\ $\mathbb{C}^{N \times r} \quad$ Espaço das matrizes complexas de ordem $N \times r$ \\ $C^{k} \quad$ Espaço das funções contínuas $k$-diferenciáveis \\ $L^{2}(\mathbb{R}) \quad$ Espaço das funções reais quadrado integráveis em $\mathbb{R}$

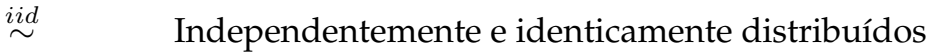 \\ $\operatorname{vec}(\cdot) \quad$ Operador que converte uma matriz num vetor coluna \\ $\otimes \quad$ Produto kronecker de matrizes \\ $\boldsymbol{\xi}(\omega) \quad$ Processo estacionário multivariado de incrementos ortogonais sobre $[-\pi, \pi]$ \\ $\mathbf{A}(u, \omega)^{*} \quad$ Transposta conjugada complexa da matriz $\mathbf{A}(u, \omega)$ \\ $\rho(A) \quad$ Posto da matriz $A$ \\ $\mathbb{I}_{k} \quad$ Matriz identidade de dimensão $k \times k$ \\ $u=t / T \quad$ Tempo re-escalado \\ $\Lambda(t / T) \quad$ Matriz $N \times r$ de cargas fatoriais variando no tempo
}


$\mathbf{Y}_{t} \quad$ Vetor $N \times 1$ de observações de $N$ séries temporais no instante $t$

Y Matriz de observações de dimensão $T \times N$

$\Gamma_{Y}(t / T) \quad$ Matriz $N \times N$ de covariância de $\mathbf{Y}_{t}$

$\mathbf{F}_{t} \quad$ Vetor $r \times 1$ de fatores no instante $t$

$\tilde{\mathbf{F}}_{t} \quad$ Vetor $r \times 1$ de fatores estimados no instante $t$

F Matriz de fatores comuns de dimensão $T \times r$

$\Gamma_{F} \quad$ Matriz $r \times r$ de covariância de $\mathbf{F}_{t}$

e etor $_{t} \times 1$ de componentes idiossincráticas no instante $t$

e Matriz de erros idiossincráticos de dimensão $T \times N$

$\Gamma_{e} \quad$ Matriz de covariância de $\mathbf{e}_{t}$

$\mathbf{w}_{t} \quad$ Vetor $N \times 1$ de erros no instante $t$

w Matriz de erros de dimensão $T \times N$

$\Gamma_{w} \quad$ Matriz $N \times N$ de covariância de $\mathbf{w}_{t}$

$\mathbf{X}_{t} \quad$ Vetor $N \times 1$ de componentes comuns no instante $t$

$\Gamma_{X}(t / T) \quad$ Matriz $N \times N$ de covariância de $\mathbf{X}_{t}$ 


\section{Capítulo 1}

\section{Introdução}

Os modelos fatoriais são extensivamente usados como um recurso relevante para reduzir a dimensionalidade e para explicar a estrutura de variância comum em conjuntos de dados multivariados. No contexto de séries temporais, estes problemas são de interesse, uma vez que, por exemplo, nos modelos VARMA e M-GARCH o número de parâmetros cresce conforme o número de séries consideradas no modelo, assim a análise da estrutura de covariância se torna mais difícil, especialmente quando as séries temporais são não estacionárias de segunda ordem, apresentam co-movimentos, transições no tempo e mudanças estruturais.

Para ilustrar evidência empírica em conjuntos de dados econômicos que mostram essas características, observamos a estrutura de taxas de juros de um conjunto de séries temporais macroeconômicas nos Estados Unidos, desde janeiro de 1960 até dezembro de 2003 (528 observações mensais do log-retorno). As séries temporais selecionadas se mostram na Figura 1.1, juntamente com o seu desvio padrão ${ }^{1}$. Notemos que co-movimentos e mudanças estruturais ocorrem aproximadamente nos mesmos períodos para todas as séries, dando evidência de que um possível modelo para sua descrição e redução da dimensionalidade é através de modelos com parâmetros variando no tempo.
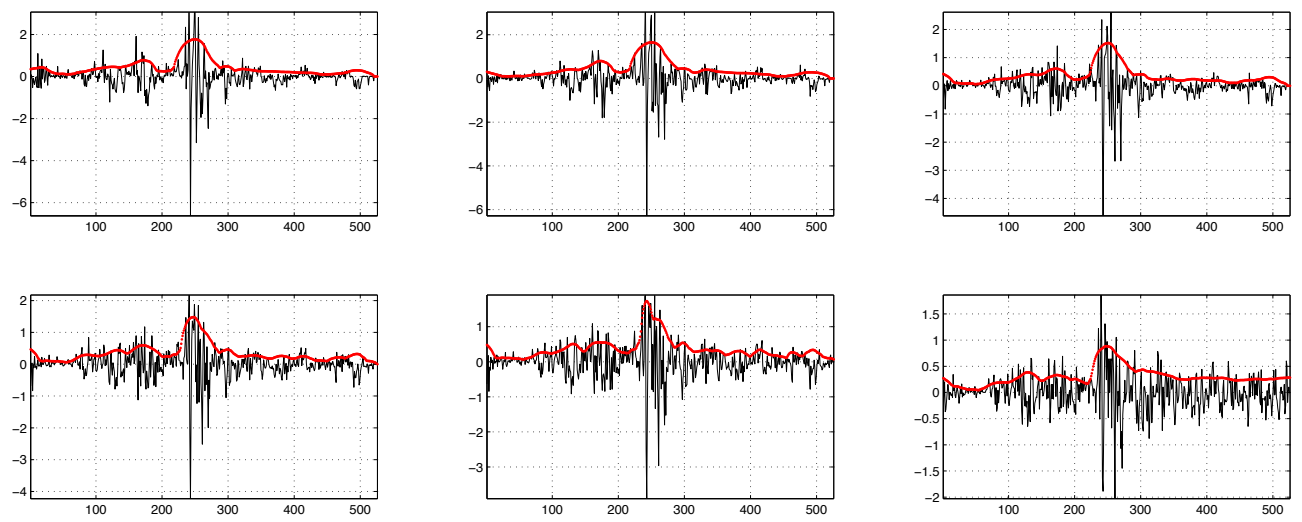

Figura 1.1: Taxas de juros mensais (preto) e sua volatilidade variando no tempo (vermelho). Motta et al. (2011).

Recentemente diversas metodologias para lidar com as mudanças estruturais nas cargas nos modelos fatoriais são apresentadas na literatura. Stock e Watson (2009) examinaram a confiabilidade de previsão

\footnotetext{
${ }^{1}$ Neste caso o desvio padrão marginal foi calculado usando função kernel com uma janela de tamanho fixo
} 
quando há uma ruptura estrutural nas cargas fatoriais. Motta et al. (2011) consideraram variações suaves nas cargas fatoriais e apresentaram um procedimento de estimação local baseado em funções kernel no domínio do tempo. Han e Inoue (2015) propuseram um teste para quebras estruturais nas cargas, verificando se os segundos momentos dos fatores estimados exibem mudanças ao longo do tempo. Cheng et al. (2016) consideraram o caso em que as cargas fatoriais e o número de fatores podem mudar simultaneamente em um determinado instante. Por outro lado, Mikkelsen et at. (2016) propuseram um modelo fatorial em que as cargas evoluem conforme uma estrutura dinâmica VAR, em que o processo de estimação das cargas é efetuado empregando procedimentos baseados em filtro de Kalman e na máxima verossimilhança.

Alguns destes trabalhos forneceram ferramentas adequadas para examinar o problema de mudanças estruturais nas cargas fatoriais. No entanto, muitos deles focalizaram no caso de variações estruturais abruptas. Essas análises podem ser inadequadas se tais mudanças nas transições, por exemplo, de preferências, progresso tecnológico ou de políticas, desempenham um papel significativo ao longo do tempo. De fato, como destaca Hansen (2001), "it may seem unlikely that a structural break could be immediate and might seem more reasonable to allow a structural change to take a period of time to take effect". Por isso, pensamos que é mais realista assumir variações suaves em lugar de variações abruptas.

Neste trabalho, queremos modelar as mudanças estruturais suaves nas cargas fatoriais ao longo do tempo através de funções de ondaletas. Pensamos que tal situação é realista na análise econômica e financeira, pois as forças motrizes podem induzir variações evolutivas suaves e seus efeitos cumulativos não podem ser simplesmente ignorados. Para capturar essas variações evolutivas suaves, consideramos um modelo fatorial da forma

$$
\mathbf{Y}_{t}=\boldsymbol{\Lambda}(t / T) \mathbf{F}_{t}+\mathbf{e}_{t}
$$

em que $\mathbf{Y}_{t}$ é um vetor de $N$ séries observadas no instante $t$ de dimensão $N \times 1$, para $t=1,2, \ldots, T$, e cada componente deste vetor depende de $r$ fatores comuns não observáveis $\mathbf{F}_{t}$ com dimensão $r \times 1$, sendo $r<<N$, tal que os componentes comuns

$$
\mathbf{X}_{t}=\boldsymbol{\Lambda}(t / T) \mathbf{F}_{t}
$$

são localmente estacionários no sentido de Dahlhaus (1997). Com o objetivo de capturar tais mudanças estruturais conjuntas no vetor de séries temporais, os componentes da matriz de cargas fatoriais são considerados como funções determinísticas suaves e contínuas no tempo. O procedimento de estimação consiste em dois estágios. No primeiro, os fatores comuns são estimados empregando os CP das séries observadas, e num segundo estágio, tratamos estes CP como co-variáveis e as cargas como coeficientes funcionais pertencentes ao espaço $\mathcal{F}$, definido em (3.28) no Capítulo 3. Posteriormente obtemos um estimador de MQ generalizados dos coeficientes das expansões de ondaletas das cargas fatoriais.

Permitindo mudanças suaves nas cargas e controlando a magnitude das instabilidades, provamos sob algumas suposições, que os estimadores dos fatores através dos CP são consistentes. Aqui assumi- 
mos que os fatores são serialmente independentes e não dependem dos erros, e a matriz de covariância dos erros idiossincráticos é positiva definida, permitindo somente correlação na seção transversal. Por fim, algumas propriedades assintóticas do estimador de MQ dos coeficientes de ondaletas, assim como a identificabilidade são tratados.

Com a finalidade de estruturar as idéias organizamos a tese da seguinte forma. No Capítulo 2 apresentamos algumas noções básicas e uma revisão de alguns trabalhos relacionados. No Capítulo 3 introduzimos o Modelo Fatorial com Cargas Funcionais e suas propriedades. Algumas simulações para ilustrar a metodologia proposta são apresentadas no Capítulo 4. Por fim, aplicações aos dados reais é realizada no Capítulo 5. Conclusões e trabalhos futuros estão no Capítulo 6. 


\section{Capítulo 2}

\section{Noções Básicas}

Neste capítulo, descrevemos brevemente os conceitos de processo localmente estacionário, análise de ondaletas e alguns modelos fatoriais clássicos e dinâmicos em séries temporais.

\subsection{Processos Localmente Estacionários Multivariados}

Nesta seção, apresentamos a definição de processos localmente estacionários multivariados, os quais nos permite estudar a variância comum mediante suas funções de transferência assim como suas propriedades.

Definição 2.1.1. (Dahlhaus, 2000). Uma sequência de processos estocásticos multivariados $N$ - dimensional com média zero, $\boldsymbol{X}_{t}=\left(X_{1 t}, \ldots, X_{N t}\right)^{\prime}$, é chamada localmente estacionária com função de transferência $\boldsymbol{A}_{T}^{0}(t, \omega)$, $\omega \in[-\pi, \pi]$, se existe uma representação

$$
\boldsymbol{X}_{t}=\int_{-\pi}^{\pi} e^{i \omega t} \boldsymbol{A}_{T}^{0}(t, \omega) d \boldsymbol{\xi}(\omega)
$$

com as seguintes propriedades

a. $\boldsymbol{\xi}(\omega)=\left(\xi_{1}(\omega), \ldots, \xi_{r}(\omega)\right)^{\prime}$ é um processo estocástico sobre $[-\pi, \pi]$ com $\overline{\xi_{i}(\omega)}=\xi_{i}(-\omega), i=1, \ldots, r$, $\mathbb{E}[\boldsymbol{\xi}(\omega)]=\mathbf{0} e$

$$
\mathbb{E}\left[d \xi_{i}(\omega) d \xi_{j}(\mu)\right]=\delta_{i j} \eta(\omega+\mu) d \omega d \mu,
$$

em que $\eta(\omega)=\sum_{j=-\infty}^{\infty} \delta(\omega+2 \pi j)$ é a extensão da função delta Dirac de periodo $2 \pi$.

b. Existe uma constante $K$ e uma função com valores matriciais $2 \pi$-periódica $A:[0,1] \times \mathbb{R} \rightarrow \mathbb{C}^{N \times r}$ com $A(u,-\omega)=\overline{A(u, \omega)} e$

$$
\sup _{t, \omega}\left|A_{i j T}^{0}(t, \omega)-A_{i j}\left(\frac{t}{T}, \omega\right)\right| \leq \frac{K}{T}
$$

para todo $i=1, \ldots, N, j=1, \ldots$, r e $T \in \mathbb{N} ; \boldsymbol{A}(u, \omega)$ é assumida contínua em $u$. 
A suavidade de A em $u$ garante que o processo tenha um comportamento localmente estacionário. A função matricial de ordem $N \times N$

$$
\Gamma(u, \omega)=\mathbf{A}(u, \omega) \mathbf{A}(u, \omega)^{*},
$$

é chamada densidade espectral variante no tempo do processo, em que $\mathbf{A}(u, \omega)^{*}$ denota a transposta conjugada complexa da matriz $\mathbf{A}(u, \omega)$. Ela é definida, levando em consideração a função de transferência definida em (2.1), como a matriz limite obtida quando $T \rightarrow \infty$ se a função $\mathbf{A}(u, \omega)$ em (2.4) é substituída por $\mathbf{A}_{T}^{0}(t, \omega)$, com $t=[u T]$. Dahlhaus (1996a, Teorema 2.2) provou que $\Gamma(u, \omega)$ é unicamente determinada por um arranjo triangular.

Observação 2.1.1. Aqui consideramos a definição de processo localmente estacionário Gaussiano com média zero. Podemos considerar a definição a partir de um processo $\xi$ não Gaussiano baseado nos cumulantes, mas para os objetivos do trabalho é mais relevante a convergência em (2.3).

\section{Processos Evolutivos}

A seguir apresentamos dois casos particulares do processo localmente estacionário, o processo tempo modulado e processo linear evolutivo. Estes dois processos são importantes para definir os componentes comuns dos modelos fatoriais estáticos e dinâmicos.

Definição 2.1.2. (Priestley, 1981). Seja $\boldsymbol{F}_{t}$ um processo estacionário $r$ - dimensional com representação espectral

$$
\boldsymbol{F}_{t}=\int_{-\pi}^{\pi} e^{i \omega t} \boldsymbol{A}(\omega) d \boldsymbol{\xi}(\omega)
$$

com função de transferêcia matricial $\boldsymbol{A}(\omega)$ de ordem $r \times r$ e $\boldsymbol{\xi}(\omega)=\left(\xi_{1}(\omega), \ldots, \xi_{r}(\omega)\right)^{\prime}$ um processo estocástico com incrementos ortogonais sobre $[-\pi, \pi]$. Sejam $\boldsymbol{m}:[0,1] \rightarrow \mathbb{R}^{N}$ e $\boldsymbol{s}:[0,1] \rightarrow \mathbb{R}^{N \times r}$ funções contínuas. Então dizemos que

$$
\boldsymbol{X}_{t}=\boldsymbol{m}(t / T)+\boldsymbol{s}(t / T) \boldsymbol{F}_{t}
$$

é um Processo Estacionário Tempo Modulado. A variância de (2.6) é dada por

$$
\operatorname{Var}\left(\boldsymbol{X}_{t}\right)=\boldsymbol{s}(t / T) \Gamma_{F} \boldsymbol{s}(t / T)^{\prime}
$$

Proposição 2.1.1. O Processo Estacionário Tempo Modulado definido em (2.6) é um Processo Localmente Estacionário.

Na prova desta proposição, mostra-se que a função de transferência $\mathbf{A}_{T}^{0}(t, \omega)=\mathbf{A}(t / T, \omega)=\mathbf{s}(t / T) \mathbf{A}(\omega)$, satisfaz $(b)$ da Definição 2.1.1 e que o processo $\boldsymbol{\xi}(\omega)$ satisfaz $(a)$ da mesma definição. Para prova com mais detalhes ver Motta et al. (2011). 
Se identificamos sua correspondente função de transferência matricial $\mathbf{A}_{T}^{0}(t, \omega)$, é possível estimar a matriz de covariância do processo (2.6) através de (2.4),

$$
\Gamma_{X}(u, \omega)=\mathbf{A}_{T}^{0}(t, \omega) \mathbf{A}_{T}^{0}(t, \omega)^{\prime}=[\mathbf{s}(t / T) \mathbf{A}(\omega)][\mathbf{s}(t / T) \mathbf{A}(\omega)]^{\prime},
$$

em que a quantidade $\mathbf{A}(\omega)$ corresponde ao desvio padrão $\Gamma_{F}^{1 / 2}$ do processo $\mathbf{F}_{t}$.

Definição 2.1.3. (Priestley, 1981). Dizemos que $X_{t}$ é um Processo Linear Evolutivo se ele pode ser representado por

$$
\boldsymbol{X}_{t}=\sum_{k=0}^{\infty} \boldsymbol{s}_{k}(t / T) \boldsymbol{F}_{t-k}
$$

com $\boldsymbol{F}_{t}$ um processo estacionário tal que $\boldsymbol{F}_{t} \sim$ id $\left(0, \Gamma_{F}\right), \boldsymbol{s}_{k}:[0,1] \rightarrow \mathbb{R}^{N \times r}$ contínua para $k \in \mathbb{N}_{0} e \sum_{k=0}^{\infty}\left\|\boldsymbol{s}_{k}\left(\frac{t}{T}\right)\right\|<$ $\infty$, com $t=1, \ldots, T$.

Kim (1999) forneceu alguns exemplos de modelos evolutivos de séries temporais econômicas, tais como, modelos evolutivos $\operatorname{VAR}(p)$, processo com raiz unitária e erros evolutivos, processos cointegrados I(1) com erros evolutivos, entre outros.

Proposição 2.1.2. O Processo Linear Evolutivo definido em (2.9) é um Processo Localmente Estacionário.

Prova. Ver apêndice

Segundo a equação (2.4), para este processo, a matriz de covariância pode ser obtida através de

$$
\Gamma_{X}(u, \omega)=\mathbf{A}_{T}^{0}(t, \omega) \mathbf{A}_{T}^{0}(t, \omega)^{*}
$$

com

$$
\mathbf{A}_{T}^{0}(t, \omega)=(2 \pi)^{-1 / 2} \sum_{k=0}^{\infty} s_{k}\left(\frac{t}{T}\right) \Gamma_{F} e^{-i \omega k},
$$

em que $\boldsymbol{s}_{k}\left(\frac{t}{T}\right)$ são matrizes de ordem $N \times r$ e $\Gamma_{F}$ é a matriz de covariância $r \times r$ do processo $\mathbf{F}_{t}=$ $\left(F_{1 t}, F_{2 t}, \ldots, F_{r t}\right)^{\prime}$.

Assim estes dois tipos de processos são considerados, casos particulares dos processos localmente estacionários, em que a função de transferência $\mathbf{A}_{T}^{0}(t, \omega)$ na representação espectral coincide com a função em que a densidade espectral $\Gamma(u, \omega)$ é definida, isto é, $\mathbf{A}_{T}^{0}(t, \omega)=\mathbf{A}\left(\frac{t}{T}, \omega\right)$ para todo $t=1, \ldots, T$, $T \in \mathbb{N}$ e $\omega \in[-\pi, \pi]$.

\subsection{Ondaletas}

Nesta seção são apresentados alguns conceitos básicos sobre a teoria de ondaletas como uma ferramenta para aproximação de funções. Uma das características importantes das ondaletas é a localização em tempo-escala e sua adaptabilidade em espaços não-homogêneos como os de Besov (Donoho e Johnstone, 1994), permitindo a análise de processos não estacionários. 


\section{Funções Ondaletas}

O propósito da teoria das ondaletas é gerar espaços a partir de dilatações e translações de uma única função $\psi$, denominada ondaleta mãe. Assim, desejamos aproximar funções de $L^{2}(\mathbb{R})$ utilizando combinações lineares de funções pertencentes a este espaço. A base de funções geradas pertence à classe

$$
\Psi=\left\{\psi_{a b}(x)=|a|^{-1 / 2} \psi\left(\frac{x-b}{a}\right) ; a, b \in \mathbb{R}, a \neq 0\right\}
$$

em que a ondaleta mãe $\psi$ deve satisfazer as seguintes propriedades
a. $\int_{-\infty}^{\infty} \psi(t) d t=0$ (admissibilidade),
b. $\int_{-\infty}^{\infty}|\psi(t)| d t<\infty$,
c. $\int_{-\infty}^{\infty} \frac{|\hat{\psi}(\lambda)|}{|\lambda|} d \lambda<\infty, \hat{\psi}(\lambda)$ é a transformada de Fourier de $\psi(t)$,
d. $\int_{-\infty}^{\infty} t^{j} \psi(t) d t=0, \quad j=0,1, \ldots, r-1$, para algum $r>0$ e $\int_{-\infty}^{\infty}\left|t^{r} \psi(t)\right| d t<\infty$.

Considere uma base ortonormal gerada por $\psi$, através de dilatações binárias e translações diádicas, da forma

$$
\psi_{j k}(t)=2^{j / 2} \psi\left(2^{j} t-k\right),
$$

em que $j, k \in \mathbb{Z}$. Como resultado temos que para toda função $\lambda(t) \in L^{2}(\mathbb{R})$, pode ser representada através da expansão em ondaletas como

$$
\lambda(t)=\sum_{j=-\infty}^{\infty} \sum_{k=-\infty}^{\infty} \beta_{j k} \psi_{j k}(t)
$$

em que a convergência é dada em termos de média quadrática, e os coeficientes de ondaletas $\beta_{j k}$ são obtidos através do seguinte produto interno

$$
\beta_{j k}=<\lambda, \psi_{j k}>=\int_{-\infty}^{\infty} \lambda(t) \psi_{j k}(t) d t
$$

Como o produto interno não depende do domínio no qual é aplicado, analogamente à análise de Fourier, a relação de Parseval da energia é válida, ou seja,

$$
\int_{-\infty}^{\infty} \lambda(t)^{2} d t=\sum_{j=-\infty}^{\infty} \sum_{k=-\infty}^{\infty} \beta_{j k}^{2}
$$

Visto que cada coeficiente $\beta_{j k}$ está associado a uma função $\psi_{j k}$ localizada no tempo-escala (índices $j$ e $k$ ), é possível obter um mapeamento da energia do sinal tanto temporalmente quanto na escala. Em geral, as ondaletas não têm estruturas analíticas fechadas como as Daubechies (D8). Um caso particular é a ondaleta Haar, que possui forma fechada 


$$
\psi(t)=\left\{\begin{array}{rll}
1 & , \quad 0 \leq t<1 / 2 \\
-1 & , \quad 1 / 2 \leq t<1 \\
0 & , \quad \text { c.c. }
\end{array}\right.
$$

A Figura 2.1 apresenta a função Haar e D8 como um exemplo.

Haar

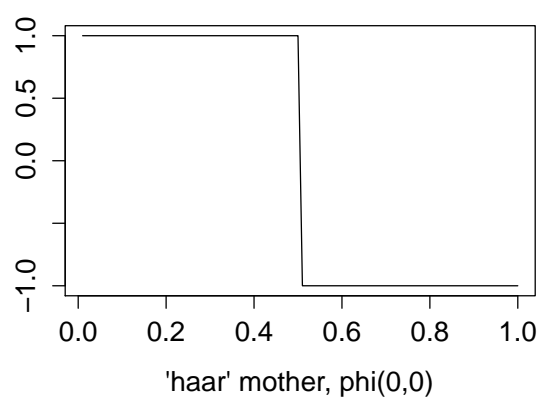

Daubechies 8

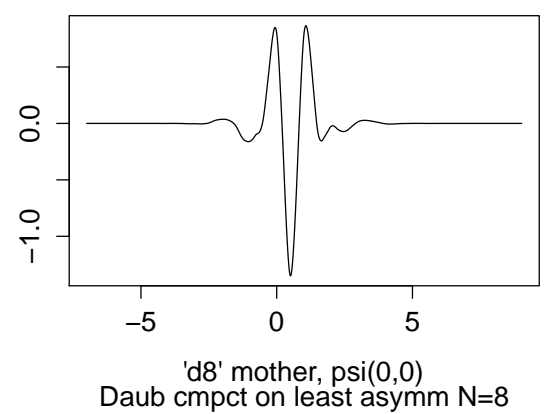

Figura 2.1: Ondaletas Haar e Daubechies D8.

Outra forma gerar ondaletas ortonormais, é considerar funções ortogonais $\phi$ solução da equação

$$
\phi(t)=\sqrt{2} \sum_{k} l_{k} \phi(2 t-k) d t
$$

em que

$$
l_{k}=\sqrt{2} \int_{-\infty}^{\infty} \phi(t) \phi(2 t-k) d t .
$$

Como no caso das funções $\psi$, através de dilatações e translações ortonormais desta $\phi$, uma família ortonormal é gerada da forma

$$
\phi_{j k}(t)=2^{j / 2} \phi\left(2^{j} t-k\right) d t, \quad j, k \in \mathbb{Z} .
$$

Portanto, é possível obter funções $\psi$ que satisfazem todas as propriedades descritas anteriormente, através de um filtro em $\phi$, como

$$
\psi(t)=\sqrt{2} \sum_{k} h_{k} \phi(2 t-k),
$$

com

$$
h_{k}=\sqrt{2} \int_{-\infty}^{\infty} \psi(t) \phi(2 t-k) d t,
$$

em que temos a quadrature mirror filter relation dada por $h_{k}=(-1)^{k} l_{1-k}$. Se observamos os filtros $l_{k}$ e $h_{k}$ no enfoque de análise de Fourier, $l_{k}$ é considerado como um filtro passa-baixa e $h_{k}$ um filtro passa-alta. Além disso, podemos definir um sistema ortonormal da forma

$$
\left\{\psi_{j k}, \phi_{j k} ; j, k \in \mathbb{Z}\right\} .
$$


Assim, dados os coeficientes dos filtros, qualquer função $\lambda(t) \in L^{2}(\mathbb{R})$, pode ser escrita através da expansão em ondaletas dada por

$$
\lambda(t)=\sum_{k} \alpha_{j_{0} k} \phi_{j_{0} k}(t)+\sum_{j \geq j_{0}} \sum_{k} \beta_{j k} \psi_{j k}(t)
$$

com os coeficientes da expansão são dados por

$$
\alpha_{j_{0} k}=\int_{-\infty}^{\infty} \lambda(t) \phi_{j_{0} k}(t) d t, \quad \beta_{j k}=\int_{-\infty}^{\infty} \lambda(t) \psi_{j k}(t) d t
$$

Frequentemente na prática $j_{0}$ é tomado como zero. As ondaletas mais utilizadas são as ondaletas com suporte compacto, tais como Haar, Daublets, Symlet e Daubechies, construídas através dos filtros passabaixa.

\section{Análise Multirresolução}

De acordo com Cohen e Ryan (1995), uma Análise Multirresolução (AMR), é uma ferramenta onde podemos observar os dados em diferentes resoluções, permitindo desprezar ou incluir detalhes. Mais formalmente, uma AMR é uma sequência crescente de espaços fechados $V_{i}$, com as seguintes propriedades
a. $\ldots \subset V_{-1} \subset V_{0} \subset V_{1} \subset \ldots$,
b. $L^{2}(\mathbb{R})=\overline{\cup_{j} V_{j}}$,
c. $\cap_{j} V_{j}=\{0\}$,
d. $\lambda(t) \in V_{j}$ se e somente se $\lambda(2 t) \in V_{j+1}$,
e. $V_{j+1}=V_{j} \oplus W_{j}, \quad W_{j} \perp V_{j}$.

Seja $\lambda(t) \in L^{2}(\mathbb{R})$ e desejamos obter uma aproximação desta função em vários níveis de resolução. Cada um dos subespaços $V_{i}$ conterá funções aproximantes, e a aproximação com menor distância é dada pela projeção ortogonal de $\lambda(t)$ em cada um desses subespaços. Assim, como $V_{i} \subset V_{i+1}$, se passarmos a projeção de $V_{i}$ para $V_{i+1}$ adicionamos detalhes à aproximação da função. Além disso, da propriedade (e), se verifica que os detalhes estão em um espaço ortogonal anterior. Pode-se mostrar, que o sistema $\left\{\psi_{j k}, k \in \mathbb{Z}\right\}$ forma uma base ortogonal para $W_{j}$ e $\left\{\phi_{j k}, k \in \mathbb{Z}\right\}$ forma uma base ortogonal para $V_{j}$.

Assim, podemos verificar a seguinte relação:

$$
L^{2}(\mathbb{R})=V_{j_{0}} \bigoplus\left(\bigoplus_{j \geq j_{0}} W_{j}\right),
$$

com $j_{0} \in \mathbb{Z}$. Dependendo da escolha de $j_{0}$, podemos separar a função em um termo da resolução e outro de detalhes, ver por exemplo, Cohen e Ryan (1995), Daubechies (1992) e Mallat (2008). 


\section{A transformada de Ondaletas}

Grossmann e Morlet (1985) são os trabalhos referentes da transformada contínua de ondaletas. A transformada contínua de ondaleta da função $\lambda(t) \in L^{2}(\mathbb{R})$ é definida pelo seguinte produto interno

$$
W_{\psi}(a b)=<\lambda, \psi_{a b}>=|a|^{-1 / 2} \int_{-\infty}^{\infty} \lambda(t) \psi_{a b}\left(\frac{t-b}{a}\right) d t
$$

com $a, b \in \mathbb{R}, a \neq 0$. Porém, na prática, a maioria das séries temporais e processos estocásticos se apresentam na forma discreta $\mathbf{X}_{T}=\left(x_{0}, x_{1}, \ldots, x_{T-1}\right)$. Assumamos que $T=2^{J}$ e $J \in \mathbb{N}$, a transformada de ondaleta discreta de $\mathbf{X}_{T}$ com respeito à função geradora $\psi$ é definida por

$$
\beta_{j k}=\sum_{t=0}^{T-1} x_{t} \psi_{j k}(t)
$$

a qual é calculada para $j=0,1, \ldots, J-1$ e $k=0,1, \ldots, 2^{j}-1$. Portanto pela relação (2.26), tomando $j_{0}=0$

$$
x_{t}=\alpha_{00} \phi(t)+\sum_{j=0}^{J-1} \sum_{k=0}^{2^{j}-1} \beta_{j k} \psi_{j k}(t),
$$

em que $\phi(t)$ é a função escala ou ondaleta pai.

Na prática, a transformada de ondaleta discreta não é calculada empregando diretamente (2.29), se aplica o algoritmo piramidal, que baseia-se na construção de ondaletas utilizando os filtros passa-alta $\left(h_{k}\right)$ e passa-baixa $\left(l_{k}\right)$, como é descrito em Mallat (1989). Para mais detalhes ver Morettin (2014). 


\subsection{Modelos Fatoriais com Cargas Estáticas}

A idéia intuitiva dos modelos fatoriais é descrever um conjunto grande de variáveis por um número menor de fatores comuns. A necessidade de reduzir a dimensão das variáveis a um espaço fatorial de dimensão menor é compreensível quando o conjunto de dados tratado é de grande dimensão. Por exemplo, muitas séries temporais exibem características semelhantes, tais como tendências comuns ou variâncias comuns, que podem ser representados por poucos fatores. A seguir, apresentamos alguns modelos fatoriais comumente utilizados no contexto de séries temporais multivariadas.

\section{Modelo Fatorial Ortogonal}

O modelo mais simples é o modelo fatorial ortogonal, em que o vetor de séries $\mathbf{Y}_{t}=\left(Y_{1 t}, Y_{2 t}, \ldots, Y_{N t}\right)^{\prime}$, $t=1, \ldots, T$, é conduzido por um número menor de fatores comuns. Esse modelo pode ser representado através da equação

$$
\mathbf{Y}_{t}=\Lambda \mathbf{F}_{t}+\mathbf{e}_{t}
$$

em que $\boldsymbol{\Lambda}$ é uma matriz de cargas de ordem $N \times r, \mathbf{F}_{t}=\left(F_{1 t}, F_{2 t}, \ldots, F_{r t}\right)^{\prime}$ é um vetor de fatores comuns $r$-dimensional com $r<<N$, e $\mathbf{e}_{t}$ uma sequência de vetores aleatórios com média zero e $\operatorname{Var}\left(\mathbf{e}_{t}\right)=\Gamma_{e}=$ $\operatorname{diag}\left\{\gamma_{1}^{2}, \gamma_{2}^{2}, \ldots, \gamma_{N}^{2}\right\}$. Na equação (2.30), tanto $\mathbf{F}_{t}$ quanto $\mathbf{e}_{t}$ são independentes com $\mathbb{E}\left(\mathbf{F}_{t}\right)=\mathbf{0}$ e $\operatorname{Var}\left(\mathbf{F}_{t}\right)=$ $\mathbb{I}_{r}$, assim $\mathbb{E}\left(\mathbf{Y}_{t}\right)=\mathbf{0}$. A matriz de cargas fatoriais $\boldsymbol{\Lambda}$ é assumida de posto completo que corresponde ao número de fatores comuns. Com as suposições descritas anteriormente e a estacionariedade de $\mathbf{Y}_{t}$, a matriz de autocovariância satisfaz

$$
\begin{array}{ll}
\Gamma_{Y}(0)=\boldsymbol{\Lambda} \Gamma_{F}(0) \boldsymbol{\Lambda}^{\prime}+\Gamma_{e}=\boldsymbol{\Lambda} \boldsymbol{\Lambda}^{\prime}+ & \Gamma_{e}, \\
\Gamma_{Y}(h)=\boldsymbol{\Lambda} \Gamma_{F}(h) \boldsymbol{\Lambda}^{\prime}, & h>0,
\end{array}
$$

em que as $\Gamma_{Y}(h)$ denotam matrizes de autocovariância com defasagem $h \geq 0$. Pela equação (2.31), as matrizes de autocovariância de um modelo fatorial ortogonal $\mathbf{Y}_{t}$ são todas singulares para $h>0$, assim o número de fatores comuns $r$ é o máximo dos postos de $\Gamma_{Y}(h)$. Métodos estatísticos para explorar as propriedades de (2.31) foram propostos por Peña e Box (1987) e Geweke (1977). Um ponto fraco desta aproximação, é a exigência de estacionaridade para $\mathbf{Y}_{t}$. Peña e Poncela (2006) estenderam o modelo incluindo séries não estacionárias com raiz unitária, assim como Correal e Peña (2008) para séries temporais que apresentem comportamentos não lineares do tipo limiar (threshold).

A estimação dos modelos fatoriais ortogonais (2.30) pode ser abordada de duas maneiras. A primeira é aplicar ACP ao vetor de séries $\mathbf{Y}_{t}$ e escolher os primeiros $r$ P correspondentes aos maiores autovalores como os fatores comuns. A partir da decomposição espectral,

$$
\Gamma_{Y}(0)=\lambda_{1}^{2} \mathbf{v}_{1} \mathbf{v}_{1}^{\prime}+\lambda_{2}^{2} \mathbf{v}_{2} \mathbf{v}_{2}^{\prime}+\ldots+\lambda_{N}^{2} \mathbf{v}_{N} \mathbf{v}_{N}^{\prime}
$$


em que $\lambda_{1}^{2}, \lambda_{2}^{2}, \ldots, \lambda_{N}^{2}$ são os autovalores da matriz de covariância $\Gamma_{Y}(0)$ e $\mathbf{v}_{i}$ é o autovetor associado com o autovalor $\lambda_{i}^{2}$ e satisfaz $\left\|\mathbf{v}_{i}\right\|=1$. Suponha que os $r$ primeiros autovalores são significativos em relação aos restantes, empregando, por exemplo, o scree plot, assim podemos obter a aproximação

$$
\Gamma_{Y}(0) \approx\left[\lambda_{1} \mathbf{v}_{1}, \lambda_{2} \mathbf{v}_{2}, \ldots, \lambda_{r} \mathbf{v}_{r}\right]\left[\begin{array}{c}
\lambda_{1} \mathbf{v}_{1}^{\prime} \\
\lambda_{2} \mathbf{v}_{2}^{\prime} \\
\vdots \\
\lambda_{r} \mathbf{v}_{r}^{\prime}
\end{array}\right]+\hat{\Gamma}_{e} \equiv \hat{\Lambda} \hat{\Lambda}^{\prime}+\hat{\Gamma}_{e}
$$

em que $\hat{\Gamma}_{e}=\operatorname{diag}\left\{\gamma_{1}^{2}, \gamma_{2}^{2}, \ldots, \gamma_{N}^{2}\right\}=\operatorname{diag}\left[\Gamma_{Y}(0)\right]-\operatorname{diag}\left[\hat{\mathbf{\Lambda}} \hat{\mathbf{\Lambda}}^{\prime}\right]$. Tradicionalmente, a escolha de $r$ é determinado subjetivamente observando o "scree plot" dos autovalores de $\Gamma_{Y}(0)$. Para mais detalhes do uso de ACP em análise fatorial, ver Johnson e Wichern (2007), Peña e Box (1987) e Bai e Ng (2013). A segunda aproximação é usar estimação de máxima verossimilhança sob normalidade nos erros e as suposições de regularidade: (a) $\Gamma_{e}$ é não singular e (b) $\Lambda^{\prime} \Gamma_{e}^{-1} \Lambda$ é uma matriz diagonal. Este método também exige que o número de fatores $r$ seja conhecido.

\section{Modelo Fatorial Aproximado}

O requerimento para que a matriz de covariância dos erros idiossincráticos $\mathbf{e}_{t}$ seja diagonal na equação (2.30) pode ser difícil de atingir quando a dimensão $N$ de $\mathbf{Y}_{t}$ é grande. Por essa razão, Chamberlain e Rothschild (1983) propuseram os modelos fatoriais aproximados, os quais permitem uma estrutura mais geral na matriz de covariância $\Gamma_{e}$. Consideremos um modelo fatorial aproximado

$$
\mathbf{Y}_{t}=\Lambda \mathbf{F}_{t}+\mathbf{e}_{t}
$$

em que $\mathbf{e}_{t}$ satisfaz (a) $\mathbb{E}\left(\mathbf{e}_{t}\right)=0$, (b) $\operatorname{Var}\left(\mathbf{e}_{t}\right)=\Gamma_{e}$, e (c) $\operatorname{Cov}\left(e_{i t}, e_{i s}\right)=0$ para $t \neq s$. Uma desvantagem deste modelo é que, para $N<\infty$, não é unicamente identificado. Por exemplo, assuma que $\mathbf{F}_{t}$ não é serialmente correlacionado, seja $C$ alguma matriz ortogonal de ordem $N$ e definimos

$$
\boldsymbol{\Lambda}^{*}=C \boldsymbol{\Lambda}, \quad \mathbf{e}_{t}^{*}=\left(\mathbb{I}_{N}-C\right) \boldsymbol{\Lambda} \mathbf{F}_{t}+\mathbf{e}_{t} .
$$

Assim, o modelo pode ser reescrito como

$$
\mathbf{Y}_{t}=\mathbf{\Lambda}^{*} \mathbf{F}_{t}+\mathbf{e}_{t}^{*}
$$

o qual também é um modelo fatorial aproximado. No trabalho de Forni et al. (2000) os modelo fatoriais aproximados podem ser identificados se os $r$ maiores autovalores da matriz de covariância de $\mathbf{Y}_{t}$ divergem quando $N \rightarrow \infty$, enquanto que os autovalores da matriz de covariância dos erros permanecem limitados. Portanto, o modelo fatorial aproximado na equação (2.33) é só assintóticamente identificado. 
Por outra parte, Lam, Yao, e Bathia (2011) e Lam e Yao (2012), consideram o modelo (2.33), mas requerem que (a) $\boldsymbol{\Lambda}$ seja uma matriz de ordem $N \times r$ ortogonal, assim que $\boldsymbol{\Lambda}^{\prime} \boldsymbol{\Lambda}=\mathbb{I}_{r}$, (b) é um vetor de ruído branco com $\mathbb{E}\left(\mathbf{e}_{t}\right)=0$ e $\operatorname{Var}\left(\mathbf{e}_{t}\right)=\Gamma_{e}$ uma matriz de covariância geral, (c) $\operatorname{Cov}\left(\mathbf{e}_{t}, \mathbf{F}_{s}\right)=\mathbf{0}$ para $s \neq t$, e combinações não lineares de $\mathbf{F}_{t}$ são ruído branco. Algumas outras suposições são necessárias para fazer a estimação de $\boldsymbol{\Lambda}$ e $\mathbf{F}_{t}$; ver Lam, Yao e Bathia (2011) para mais detalhes.

\section{Modelo Fatorial Dinâmico}

Nos casos acima, não é considerado explicitamente a dependência dinâmica dos fatores comuns $\mathbf{F}_{t}$. Forni et al. (2000) propuseram o modelo fatorial dinâmico

$$
\mathbf{Y}_{t}=\mathbf{\Lambda}(B) \mathbf{a}_{t}+\mathbf{e}_{t}
$$

em que $\mathbf{e}_{t}$ é um vetor de ruído branco com média zero e matriz de covariância $\Gamma_{e}, \mathbf{a}_{t}$ é um processo $r$-dimensional de ruído branco ortonormal, e $\boldsymbol{\Lambda}(B)=\boldsymbol{\Lambda}_{0}+\boldsymbol{\Lambda}_{1} B+\ldots+\boldsymbol{\Lambda}_{m} B^{m}$ é uma matriz $N \times r$ polinômio de grau $m$ (pode ser infinito), e $B$ é o operador tal que $B \mathbf{a}_{t}=\mathbf{a}_{t-1}$. Ruído branco ortonormal significa que satisfaz $\mathbb{E}\left(\mathbf{a}_{t}\right)=\mathbf{0}, \operatorname{Cov}\left(\mathbf{a}_{t}\right)=\mathbb{I}_{m}$, e $\mathbf{a}_{t}$ é serialmente não correlacionado. Seja $\boldsymbol{\Lambda}(B)=\left[\Lambda_{i j}(B)\right]$, com $i=1, \ldots, N$ e $j=1, \ldots, r$, em que $\sum_{i=0}^{\infty}\left\|\boldsymbol{\Lambda}_{i}\right\|^{2}<\infty$, e denote $\mathbf{C}_{t}=\boldsymbol{\Lambda}(B) \mathbf{a}_{t}=\left(C_{1 t}, C_{2 t}, \ldots, C_{N t}\right)^{\prime}$. Portanto o modelo se torna

$$
\mathbf{Y}_{t}=\mathbf{C}_{t}+\mathbf{e}_{t}
$$

Forni et al. (2000) referem $C_{i t}$ e $e_{i t}$ como componente comúm e componente idiosincrática de $Y_{i t}$, respectivamente. O processo $\mathbf{a}_{t}$ é referido como choques comuns. Assumindo que $\mathbf{Y}_{t}$ da equação (2.34) é estacionário, Forni et al. (2000) empregaram a matriz de densidade espectral de $\mathbf{Y}_{t}$ para propor um método de estimação para os componentes comuns $\mathbf{C}_{t}$ quando $N, T \rightarrow \infty$. Sob condições de regularidade, se prova que as estimativas convergem para $\mathbf{C}_{t}$ em média quadrática. Os detalhes são dados em Forni et al. (2000).

Neste contexto, assumindo que os fatores comuns $\mathbf{F}_{t}$ do modelo fatorial aproximado na equação (2.33), são conduzidos por um modelo $\operatorname{VARMA}(p, q)$ estacionário

$$
\phi(B) \mathbf{F}_{t}=\boldsymbol{\theta}(B) \mathbf{a}_{t},
$$

em que $\phi(B)$ e $\boldsymbol{\theta}(B)$ são matrizes $r \times r$ polinômios AR e MA, respectivamente. Então o modelo se torna

$$
\mathbf{Y}_{t}=\boldsymbol{\Lambda}[\boldsymbol{\phi}(B)]^{-1} \boldsymbol{\theta}(B) \mathbf{a}_{t}+\mathbf{e}_{t}
$$

portanto, temos que $\boldsymbol{\Lambda}(B)=\boldsymbol{\Lambda}[\boldsymbol{\phi}(B)]^{-1} \boldsymbol{\theta}(B)$. Esta conexão entre os dois modelos oferece uma alternativa para estimar os modelos fatoriais dinâmicos. Especificamente, se os choques comuns $\mathbf{a}_{t}$ são de interesse, eles podem ser estimados primeiramente construindo um modelo fatorial aproximado para $\mathbf{Y}_{t}$, e depois modelando o processo estimado $\hat{\mathbf{F}}_{t}$, via modelos VARMA. 


\subsection{Modelo Fatorial com Cargas Variando no Tempo}

Uma das principais características dos modelos apresentados nas seções anteriores é que as cargas fatoriais permanecem fixas ao longo do tempo. Porém, isto parece restritivo, dado que durante extensos períodos de tempo pode acontecer mudanças. Por exemplo, nos casos de transições entre recessões ou impactos de crises políticas, poderia acontecer instabilidades estruturais. Como resultado é necessário introduzir parâmetros que mudam no tempo na modelagem. Apresentamos alguns modelos existentes com estimação das cargas variando no tempo, especificamente quando são estocásticas e quando são consideradas funções determinísticas do tempo.

\section{Modelo Fatorial com Cargas Estocásticas}

Para modelar as cargas variando ao longo do tempo, Mikkelsen et al. (2016) introduz uma dinâmica autorregressiva nas cargas através do seguinte modelo

$$
\mathbf{Y}_{t}=\Lambda_{t} \mathbf{F}_{t}+\mathbf{e}_{t}
$$

em que $\mathbf{Y}_{t}=\left(Y_{1 t}, Y_{2 t}, \ldots, Y_{N t}\right)^{\prime}$ é um vetor $N$-dimensional de séries temporais observadas no tempo $t$. As observações podem ser representadas por $r$ fatores comuns não observáveis $\mathbf{F}_{t}=\left(F_{1 t}, F_{2 t}, \ldots, F_{r t}\right)^{\prime}$, cargas fatoriais variando no tempo $\boldsymbol{\Lambda}_{t}$, e $\mathbf{e}_{t}=\left(e_{1 t}, e_{2 t}, \ldots, e_{N t}\right)^{\prime}$ erros idiossincráticos com matriz de covariâncias $\mathbb{E}\left(\mathbf{e}_{t} \mathbf{e}_{t}^{\prime}\right)=\Gamma_{e}$. A matriz $N \times r$ de cargas fatoriais $\boldsymbol{\Lambda}_{t}=\left(\lambda_{1 t}^{\prime}, \lambda_{2 t}^{\prime}, \ldots, \lambda_{N t}^{\prime}\right)^{\prime}$ é variante no tempo e cada $\lambda_{i t} \in \mathbb{R}^{r}$ evolui como um VAR de dimensão $r$

$$
\Phi_{i}^{0}(B)\left(\lambda_{i t}-\lambda_{i}^{0}\right)=\eta_{i t},
$$

em que $\lambda_{i}^{0}=\mathbb{E}\left(\lambda_{i t}\right)$ é a média incondicional, e a matriz $\Phi_{i}^{0}(B)=I-\Phi_{i, 1} B-\ldots-\Phi_{i, p} B^{p}$ de dimensão $r \times r$ é um polinômio autorregressivo de ordem $p$, em que as raízes de $\left|\Phi_{i}^{0}(B)\right|$, com $i=1, \ldots, N$, estão fora do círculo unitário. O vetor de erros $\eta_{i t}$ de dimensão $r \times 1$ tem matriz de covariâncias $\mathbb{E}\left(\eta_{i t} \eta_{i t}^{\prime}\right)=Q_{i}^{0}$ de $r \times r$.

Com o objetivo de estudar algumas propriedades, especificamente assintóticas, é considerada $\xi_{i t}=$ $\lambda_{i t}-\lambda_{i}^{0}=\Phi_{i}^{0}(B)^{-1} \eta_{i t}$, a representação $\mathrm{MA}(\infty)$ das cargas fatoriais, portanto o modelo (2.37) pode ser escrito como

$$
\mathbf{Y}_{t}=\Lambda^{0} \mathbf{F}_{t}+\xi_{t} \mathbf{F}_{t}+\mathbf{e}_{t}
$$

em que $\boldsymbol{\Lambda}^{0}=\left(\lambda_{1}^{0}, \lambda_{2}^{0}, \ldots, \lambda_{N}^{0}\right)^{\prime}$ e $\xi_{t}=\left(\xi_{1 t}, \xi_{2 t}, \ldots, \xi_{N t}\right)^{\prime}$ são as matrizes $N \times r$ das cargas médias e inovações, respetivamente. Os $r$ fatores comuns foram estimados via CP. As propriedades deste estimador, assim como algumas suposições de regularidade para demonstrar essas propriedades, são apresentadas em Mikkelsen et al. (2016).

O propósito é estimar os parâmetros de cada uma das cargas (2.38) e a matriz de variâncias idiossin- 
cráticas $\Gamma_{e}$ através de MV. Para a construção da função de verossimilhança, é suposto que $\Gamma_{e}=$ diag $=$ $\left\{\gamma_{1}, \gamma_{2}, \ldots, \gamma_{N}\right\}$ erros não correlacionados na seção transversal, como consequência, condicionado nos fatores, $\mathbf{Y}_{i}$ é não correlacionado sobre $i$, e pode ser escrito como

$$
\mathbf{Y}_{i}=\mathbf{F} \boldsymbol{\Lambda}_{i}+\mathbf{e}_{i}, \quad i=1, \ldots, N
$$

em que $\mathbf{Y}_{i}=\left(Y_{i 1}, Y_{i 2}, \ldots, Y_{i T}\right)^{\prime}$ é a $i$-ésima série temporal, $\mathbf{e}_{i}=\left(e_{i 1}, e_{i 2}, \ldots, e_{i T}\right)^{\prime}$ é o $i$-ésimo erro idiossincrático, $\mathbf{F}=\operatorname{diag}\left\{F_{t}^{\prime}\right\}_{t=1, \ldots, T}$ é uma matriz diagonal por blocos de $T \times r T$ e $\boldsymbol{\Lambda}_{i}=\left(\lambda_{i 1}^{\prime}, \lambda_{i 2}^{\prime}, \ldots, \lambda_{i T}^{\prime}\right)^{\prime}$. A média e a variância de $\mathbf{Y}_{i}$ são $\mathbb{E}\left(\mathbf{Y}_{i}\right)=\left(F_{1}^{\prime} \lambda_{i}^{0}, F_{2}^{\prime} \lambda_{i}^{0}, \ldots, F_{T}^{\prime} \lambda_{i}^{0}\right)^{\prime}$ e $\Gamma_{i}=\operatorname{Var}\left(\mathbf{Y}_{i}\right)=\mathbf{F} \Phi_{i} \mathbf{F}^{\prime}+\gamma_{i} I_{T}$ onde $\Phi_{i}=\operatorname{Var}\left(\boldsymbol{\Lambda}_{i}\right)$ é de dimensão $r T \times r T$.

Para o processo de estimação dos parâmetros $\theta_{i}=\left\{\Phi_{i}(B), \lambda_{i}, Q_{i}, \gamma_{i}\right\}$ é construída a função de verossimilhança gaussiana para $\mathbf{Y}_{i}$ condicionada sobre os fatores $\mathbf{F}=\left(F_{1}, F_{2}, \ldots, F_{T}\right)^{\prime}$ dada por

$$
\mathcal{L}_{T}\left(\mathbf{Y}_{i} \mid F ; \theta_{i}\right)=\frac{1}{2} \log (2 \pi)-\frac{1}{2 T} \log \left|\Gamma_{i}\right|-\frac{1}{2 T}\left[\mathbf{Y}_{i}-\mathbb{E}\left(\mathbf{Y}_{i}\right)\right]^{\prime} \Gamma_{i}^{-1}\left[\mathbf{Y}_{i}-\mathbb{E}\left(\mathbf{Y}_{i}\right)\right]
$$

para cada $i=1, \ldots, N$. Portanto, dado que os fatores F são não observáveis, eles são substituídos pelo estimador $\tilde{\mathbf{F}}$ obtido através dos $\mathrm{CP}$, e posteriormente obter $\tilde{\theta}_{i}$ que maximiza a função $\tilde{\mathcal{L}}_{T}\left(\mathbf{Y}_{i} \mid \tilde{\mathbf{F}} ; \theta_{i}\right)$,

$$
\tilde{\theta}_{i}=\underset{\theta}{\arg \max } \tilde{\mathcal{L}}_{T}\left(\mathbf{Y}_{i} \mid \tilde{\mathbf{F}} ; \theta_{i}\right)
$$

em que estimadores obtidos $\tilde{\theta}_{i}, \operatorname{com} i=1,2, \ldots, N$, são consistentes. Por fim são apresentadas diferentes suposições para provar identificabilidade assintótica ante rotações dos fatores e cargas. Alguns trabalhos referentes nesta metodologia são Mikkelsen et al. (2016), Bates et al. (2013), Breitung e Eickmeier (2011), entre outros.

\section{Modelo Fatorial Localmente Estacionário}

Para modelar as cargas fatoriais que são funções determinísticas no tempo, suponhamos que temos um processo estocástico $N$-dimensional explicado por $r$ fatores comuns, em que $r<<N$, com a seguinte estrutura

$$
\mathbf{Y}_{t}=\boldsymbol{\Lambda}(u) \mathbf{F}_{t}+\mathbf{e}_{t}
$$

com fatores $\mathbf{F}_{t}$ e componentes idiossincráticas $\mathbf{e}_{t}, t=1,2, \ldots, T$. Em (2.43) as cargas $\boldsymbol{\Lambda}(u)=\left\{\lambda_{i j}(u)\right\}, i=$ $1,2, \ldots, N ; j=1,2, \ldots, r$, são funções no tempo reescalado $u=t / T \in[0,1]$. Portanto, a matriz de covariância de $\mathbf{Y}_{t}$ é uma matriz de funções suaves no tempo

$$
\Gamma_{Y}(u)=\operatorname{Var}\left(\mathbf{Y}_{t}\right)=\mathbf{\Lambda}(u) \Gamma_{F} \mathbf{\Lambda}^{\prime}(u)+\Gamma_{e}, \quad u \in[0,1]
$$

Modelos nos quais as cargas fatoriais são consideradas como funções determinísticas no tempo, são definidos em Motta et al. (2011), como Modelos Fatoriais Localmente Estacionários (MFLE). 
Definição 2.4.1. (Motta, 2011). A sequência $\left\{\boldsymbol{Y}_{t}\right\}$ em (2.43) é um MFLE se:

a. existe $\forall_{N \in \mathbb{N}}$ uma função

$$
\boldsymbol{\Lambda}(u) \in C^{1}\left\{[0,1], \mathbb{R}^{N \times r}\right\}, \quad \rho[\boldsymbol{\Lambda}(u)]=r, \quad \forall u \in[0,1],
$$

tal que $\forall_{T}$,

$$
\Gamma_{Y}(u)=\boldsymbol{\Lambda}(u) \Gamma_{F} \boldsymbol{\Lambda}^{\prime}(u)+\Gamma_{e, N} .
$$

b. $\Gamma_{e, N}=\operatorname{Var}\left(\boldsymbol{e}_{t}\right)$ é uma sequência de matrizes positivas definidas com autovalores uniformemente limitados

$$
\sup _{N} \operatorname{val}_{1}\left(\Gamma_{e, N}\right)<\infty, \quad N \in \mathbb{N},
$$

em que val ${ }_{1}\left(\Gamma_{e, N}\right)$ denota o maior autovalor da matriz $\Gamma_{e, N}$.

Sob algumas suposições de regularidade, neste trabalho provaram que o modelo é assintoticamente identificável e o processo de estimação das cargas e fatores de (2.43) pode ser visto como a solução de otimizar a seguinte função de mínimos quadrados localmente ponderados variante no tempo

$$
L_{N T}(u ; h)=\frac{1}{N T} \sum_{s=1}^{T}\left(\mathbf{Y}_{s}-\boldsymbol{\Lambda}(s / T) \mathbf{F}_{t}\right)^{\prime}\left(\mathbf{Y}_{s}-\boldsymbol{\Lambda}(s / T) \mathbf{F}_{t}\right) K_{h}(s / T-u)
$$

sujeito a

$$
\frac{\boldsymbol{\Lambda}(s / T)^{\prime} \boldsymbol{\Lambda}(s / T)}{N}=\mathbb{I}_{r}, \quad \forall s=1,2, \ldots, T, \forall_{T \in \mathbb{N}},
$$

em que $K_{h}(\cdot)$ é a versão reescalada de uma função kernel definida sobre o tempo, para uma janela $h>0$ dada e $u \in[0,1]$. Assumindo que $h \rightarrow 0$ quando $T \rightarrow \infty$, para grandes valores de $T$ os pesos $K_{h}(s / T-u)$ só consideram as cargas $\boldsymbol{\Lambda}(s / T)$ correspondentes aos valores $s / T$ que estão perto de $u$. Motta et al. (2011) provaram que assintoticamente, as cargas que minimizam a função (2.45) só dependem de $u$.

No processo de estimação, mostraram que minimizar a função (2.45) é equivalente a maximizar

$$
N^{-2} \operatorname{tr}\left\{\boldsymbol{\Lambda}^{\prime}(u) \hat{\Gamma}_{Y}(u) \boldsymbol{\Lambda}(u)\right\},
$$

sujeito a (2.46), em que

$$
\hat{\Gamma}_{Y}(u ; h)=\frac{1}{T} \sum_{s=1}^{T} \mathbf{Y}_{s} \mathbf{Y}_{s}^{\prime} K_{h}\left(\frac{s}{T}-u\right) .
$$

Assim o conjunto de solução $\hat{\Lambda}(u)$ é $\sqrt{N}$ vezes a matriz cujas colunas são os autovetores ortonormais correspondentes aos $r$ autovalores ordenados de maior a menor da matriz $\hat{\Gamma}_{Y}(u)$ e um estimador para os fatores comuns $\hat{\mathbf{F}}_{t}=N^{-1} \hat{\boldsymbol{\Lambda}}^{\prime}(u) \mathbf{Y}_{t}$. Adicionalmente mostraram que a identificabilidade tanto das cargas quanto dos fatores é alcançada assintoticamente. Trabalhos relacionados com este tipo de estruturas são Eichler et al. (2011), Ombao e Ho (2006), entre outros, os quais realizaram um desenvolvimento similar 
para os modelos fatoriais dinâmicos, mas no domínio de frequência.

O estimador das cargas fatoriais proposto por Motta et al. (2011), apresenta bom desempenho mediante as restrições, as quais muitas vezes nas aplicações reais são difíceis de alcançar, por exemplo, matriz de cargas fatoriais ortogonal e de posto completo em cada instante de tempo, matriz de covariâncias dos erros idiosincráticos identidade e fatores independentes. Um estudo de simulação apresentado para comprovar seu desempenho mostra os resultados de estimação na Figura 2.2 sob o cenário em que $N=30, T=900, r=2, \boldsymbol{\Lambda}(t / T)=\sqrt{N} \exp \left(\pi \frac{t}{T} \mathbf{W}\right) \mathbf{A}$, onde $\mathbf{W}$ é uma matriz anti-simétrica de dimensão $N$, A é uma matriz ortogonal de ordem $r$ e $\exp \left(\pi \frac{t}{T} \mathbf{W}\right)$ é uma matriz de $N \times N$ em que o $(i, j)$-ésimo elemento é $\exp \left(\pi \frac{t}{T} w_{i j}\right)$. Então a matriz de cargas satisfaz $N^{-1} \boldsymbol{\Lambda}^{\prime}(t / T) \boldsymbol{\Lambda}(t / T)=\mathbb{I}_{r}$, para $t=1, \ldots, T$. O processo fatorial $\mathbf{F}_{t} \sim \mathcal{i i d} \mathcal{N}(0, \operatorname{diag}\{3,5 ; 1\})$ enquanto que o processo idiossincrático $\mathbf{e}_{t} \sim \mathcal{i i d} \mathcal{N}\left(0, \mathbb{I}_{N}\right)$. Observamos que as cargas verdadeiras (linhas contínuas) e estimadas (linhas pontilhada) das cargas $\lambda_{10,1}(t / T)$, $\lambda_{20,1}(t / T), \lambda_{30,1}(t / T), \lambda_{10,2}(t / T), \lambda_{20,2}(t / T)$ e $\lambda_{30,2}(t / T)$, são bem próximas.
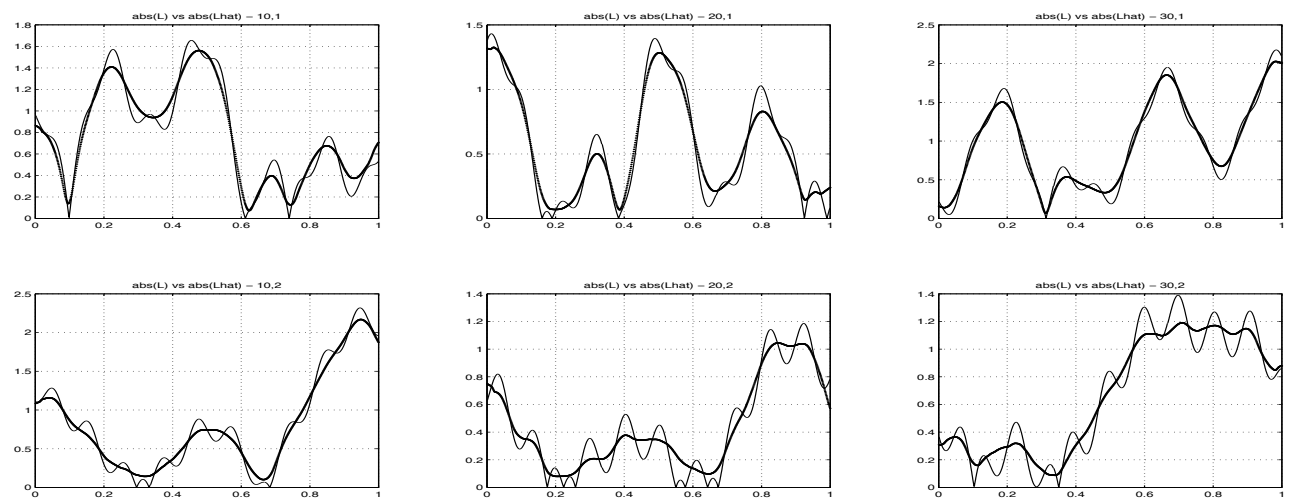

Figura 2.2: Cargas verdadeiras (linha contínua) e estimadas (linha pontilhiada).

\section{Não estacionaridade}

A não estacionaridade de um processo pode ser classificado como aleatória, determinística ou uma combinação das duas. Séries temporais não estacionárias são comumente observadas em diversas áreas, particularmente em finanças e economia, as séries temporais são caracterizadas por uma estrutura de segunda ordem que varia no tempo.

Para processos não estacionários, as propriedades assintóticas são dificilmente construídas, uma vez que, as observações futuras não contém necessariamente as mesmas informações obtidas sobre a estrutura do processo no presente e no passado. Dahlhaus (1996c, 1997) sugeriu uma abordagem baseada no conceito de tempo re-escalado, ou seja, considerar $u=t / T$. A idéia de uma teoria assintótica $(T \rightarrow \infty)$ neste contexto, é a partir de "muitas" observações $\mathbf{Y}_{t}$, estimar a estrutura local da função matricial $\Lambda(t / T)$ em cada ponto de tempo, mas no intervalo $[0,1]$, por exemplo, no seguinte modelo

$$
\mathbf{Y}_{t}=\boldsymbol{\Lambda}(t / T) \mathbf{F}_{t}+\boldsymbol{\epsilon}_{t}
$$


onde $\mathbf{Y}_{t}$ é um vetor de observações de $N \times 1$ no tempo $t$ e $\epsilon_{t}$ é um vetor de $N \times 1$ de erros no instante $t$. Observamos que o modelo (2.49) permite não estacionaridade do tipo determinístico, porque as cargas fatoriais são assumidas funções no tempo, caso que consideramos neste trabalho. 


\section{Capítulo 3}

\section{Modelo Fatorial com Cargas Funcionais}

Neste capítulo propomos um modelo fatorial com cargas funcionais e fatores estacionários. A diferença com respeito ao modelo apresentado em Motta et al. (2011), estão nas restrições da matriz de cargas funcionais, tipo dependência nos erros idiossincráticos e procedimento de estimação. Aqui propomos estimar as cargas funcionais usando funções de ondaletas e MQ generalizados, enquanto que os fatores são estimados num primeiro estágio usando os CP. Estudamos um modelo fatorial estático, no sentido que tanto os fatores quanto as cargas evoluem de forma contemporânea.

\subsection{O Modelo Proposto}

Consideramos um processo estocástico $N$-dimensional explicado por $r$ fatores $(r<N)$

$$
\begin{aligned}
& \mathbf{Y}_{t}=\mathbf{X}_{t}+\mathbf{e}_{t}, \\
& \mathbf{X}_{t}=\left[\boldsymbol{\Lambda}_{0}+\boldsymbol{\Lambda}(t / T)\right] \mathbf{F}_{t},
\end{aligned}
$$

com fatores comuns $\mathbf{F}_{t}$ e componentes idiossincráticas $\mathbf{e}_{t}$ para $t=1, \ldots, T$. A matriz de cargas fatoriais $\boldsymbol{\Lambda}(u)=\left\{\lambda_{i j}(u), \quad i=1, \ldots, N ; j=1, \ldots, r\right\}$, são funções no tempo, isto é, a influência dos fatores $\mathbf{F}_{t}$ sobre o processo observado muda ao longo do tempo, como consequência, contribue na captura de mudanças estruturais suaves; enquanto $\boldsymbol{\Lambda}_{0}$ é uma matriz de cargas constantes ${ }^{1}$. Se $\boldsymbol{\Lambda}(u)=0, \forall u \in[0,1]$, voltamos ao modelo fatorial aproximado da seção 2.3, portanto construimos uma generalização desse modelo. Os componentes comuns $\left\{\boldsymbol{X}_{t}\right\}$ são um processo localmente estacionário como foi exposto em (2.6) e as cargas são definidas no tempo re-escalado $u=t / T \in[0,1]$ como em Dahlhaus (1997). Uma explicação intuitiva para essa condição é que uma amostragem cada vez mais intensiva de pontos de dados assegura uma estimativa consistente de $\lambda(t / T)$ em algum ponto fixo $t / T$.

O processo de estimação que propomos é realizado usando funções de ondaletas para as cargas

\footnotetext{
${ }^{1}$ Neste trabalho $\boldsymbol{\Lambda}_{0}$ pode ser considerada como a matriz de cargas de referência ou equilibrio, isto é, o aporte das cargas supondo um modelo estacionário, enquanto que $\boldsymbol{\Lambda}(u)$ contribui na captura de mudanças estruturais suaves.
} 
funcionais empregando MQ generalizados, condicionado nos fatores. Estes fatores não observáveis são estimados empregando os $\mathrm{CP}$ da matriz de covariância das séries observadas como Mikkelsen et al. (2016). A seguir, caracterizamos matematicamente o modelo, ao fim de estudar suas propriedades e metodologia de estimação.

Definição 3.1.1. A sequência $\left\{\boldsymbol{Y}_{t}\right\}$ em (3.1) é um Modelo Fatorial com Cargas Funcionais se,

a. para $N \in \mathbb{N}$, existe uma função com valores matriciais

$$
\begin{aligned}
\mathbf{\Lambda}(\cdot) \quad: \quad[0,1] & \rightarrow \mathbb{R}^{N \times r} \\
u & \mapsto \boldsymbol{\Lambda}(u)
\end{aligned}
$$

tal que $\forall T \in \mathbb{N}$,

$$
\Gamma_{Y}(u)=\left[\boldsymbol{\Lambda}_{0}+\boldsymbol{\Lambda}(u)\right] \Gamma_{F}\left[\boldsymbol{\Lambda}_{0}+\boldsymbol{\Lambda}(u)\right]^{\prime}+\Gamma_{e},
$$

em que $\rho\left[\boldsymbol{\Lambda}_{0}+\boldsymbol{\Lambda}(u)\right]=\operatorname{re} \Gamma_{F}=\operatorname{Var}\left(\boldsymbol{F}_{t}\right)$,

b. $\Gamma_{e}=\operatorname{Var}\left(\boldsymbol{e}_{t}\right)$ é uma matriz positiva definida.

Nota 3.1.1. Da Proposição 2.1.1 se $\boldsymbol{F}_{t}$ é estacionário, os componentes comuns $\boldsymbol{X}_{t}=\left[\boldsymbol{\Lambda}_{0}+\boldsymbol{\Lambda}(u)\right] \boldsymbol{F}_{t}$, com $t=1, \ldots, T$, são processos localmente estacionários, permitindo estudar a variância comum assim como as propriedades assintóticas do estimador de $M Q$ generalizados dos coeficientes de ondaletas.

Nota 3.1.2. Na prática, diferentes considerações podem ser estudadas para o processo idiossincrático $N$ - dimensional $\boldsymbol{e}_{t}$. Ser conduzido por uma dinâmica VAR ou supor uma distribuição $t$-student multivariada.

\subsubsection{Suposições}

Para o modelo (3.1), seguem as suposições sobre os fatores, cargas fatoriais e erros idiossincráticos. Suponhamos que existe uma constante arbitrária $Q \in \mathbb{R}^{+}$, para as condições a seguir.

\section{S1. Fatores:}

a. $\mathbb{E}\left\|\mathbf{F}_{t}\right\|^{4}<Q$,

b. $T^{-1} \sum_{t=1}^{T} \mathbf{F}_{t} \mathbf{F}_{t}^{\prime} \stackrel{p}{\rightarrow} \Gamma_{F}$ quando $T \rightarrow \infty$, para $\Gamma_{F}$ matriz diagonal positiva definida.

\section{S2. Cargas Fatoriais:}

a. $\sup _{u \in(0,1)}\left\|\lambda_{i}(u)\right\| \leq \bar{\lambda}<\infty, \forall i$ e $\sup _{u \in(0,1)}\left\|\frac{\boldsymbol{\Lambda}^{\prime}(u) \boldsymbol{\Lambda}(u)}{N}-\Gamma_{\Lambda}(u)\right\| \rightarrow 0, N \rightarrow \infty$, em que $\lambda_{i}(u)$ é a $i$-ésima linha de $\boldsymbol{\Lambda}(u)$ e $\Gamma_{\Lambda(u)}$ é uma matriz de ordem $r \times r$ positiva definida,

b. $\left\|\lambda_{i 0}\right\| \leq \bar{\lambda}$ e $\left\|\boldsymbol{\Lambda}_{0}^{\prime} \boldsymbol{\Lambda}_{0} / N-D\right\| \rightarrow 0$, quando $N \rightarrow \infty$, com $D$ uma matriz $r \times r$ positiva definida, c. $\lambda_{i j}(u) \in \mathcal{F}$, para $i=1, \ldots, N$ e $j=1, \ldots, r$. Com $\mathcal{F}$ definido em (3.28) na Seção 3.4. 


\section{S3. Erros Idiossincráticos:}
a. $\mathbb{E}\left(e_{i t}\right)=0, \mathbb{E}\left|e_{i t}\right|^{4}<Q$,
b. $\mathbb{E}\left[e_{i t} e_{j t}\right]=\tau_{i j, t}$, com $\left|\tau_{i j, t}\right|<\left|\tau_{i j}\right|$, para $\left|\tau_{i j}\right|$ constante e $N^{-1} \sum_{i, j=1}^{N}\left|\tau_{i j}\right|<Q, \forall t$,
c. $\mathbb{E}\left|N^{-1 / 2} \sum_{i=1}^{N}\left[e_{i t} e_{i s}-\mathbb{E}\left(e_{i t} e_{i s}\right)\right]\right|^{4}<Q, \forall s, t$.

S4. Cargas Variando no Tempo e Fatores: Existe funções envelopes $K_{1 N T}, K_{2 N T}$ e $K_{3 N T}$ tal que as seguintes condições são verdadeiras para qualquer valor de $N$ e $T$, e qualquer conjunto de índices fatoriais $n, m, k, q=1, \ldots, r$. Denotemos $\lambda_{i j}(s / T) \equiv \lambda_{i j}(s)$.
a. $\sup _{s, t} \sum_{i, j}^{N}\left|\lambda_{i n}(s) \lambda_{j m}(t) \| \mathbb{E}\left[F_{n s} F_{m t}\right]\right|<K_{1 N T}$,
b. $\sum_{s, t=1}^{T} \sum_{i, j=1}^{N}\left|\lambda_{i n}(s) \lambda_{j m}(s)\right|\left|\mathbb{E}\left[F_{n s} F_{m s} F_{k t} F_{q t}\right]\right|<K_{2 N T}$,
c. $\sum_{s=1}^{T} \sum_{i, j=1}^{N}\left|\lambda_{i n}(s) \lambda_{j m}(s) \lambda_{i k}(t) \lambda_{j q}(t)\right|\left|\mathbb{E}\left[F_{n s} F_{m s} F_{k t} F_{q t}\right]\right|<K_{3 N T}$.

S5. Independência: Para quaisquer conjuntos de índices $(i, j, s, t), e_{i t}$ é independente de $F_{j s}$.

A suposição (S1.a) indica que os fatores têm quarto momento finito e sua covariância converge em probabilidade para uma matriz positiva definida. As suposições (S2.a,b) asseguram que cada fator tem uma contribuição não trivial à variância de $\mathbf{Y}_{t}$ e (S2.c), as funções $\lambda_{i j}(u) \in \mathcal{F}$, garante suavidade das cargas e existência da expansão em funções de ondaletas. Em (S3) permite-se dependência na seção transversal no processo idiossincrático. A suposição (S.4) é requerida para provar a consistência do estimador dos fatores através dos CP. Independência entre os fatores e o processo idiosincrático é dada na suposição (S5).

\subsubsection{Estimador dos Componentes Principais}

Neste trabalho usamos o estimador dos CP para estimar os fatores não observados $\mathbf{F}_{t}$, os quais são obtidos como se as cargas fatoriais fossem constantes no tempo, isto é, $\boldsymbol{\Lambda}(u) \equiv \boldsymbol{\Lambda}, \forall u \in[0,1]$, resolvendo para

$$
\min _{\mathbf{F}, \boldsymbol{\Lambda}}(N T)^{-1} \sum_{i=1}^{N} \sum_{t=1}^{T}\left(Y_{i t}-\lambda_{i}^{\prime} \mathbf{F}_{t}\right)^{2} .
$$

Denotamos $\mathbf{F}_{t}=\left(F_{1 t}, F_{2 t}, \ldots, F_{r t}\right)$ e $\mathbf{F}=\left(\mathbf{F}_{1}, \mathbf{F}_{2}, \ldots, \mathbf{F}_{T}\right)^{\prime}$ uma matriz de ordem $T \times r$ e $\boldsymbol{\Lambda}$ matriz de ordem $N \times r$. Para definir de forma única os minimizadores e eliminar problemas de indeterminacão no modelo fatorial, é preciso impor restrições de identificação sobre os estimadores, já que somente $\mathbf{Y}$ de ordem $T \times N$ é observada. Ao concentrar sobre $\boldsymbol{\Lambda}$ e empregando a padronização $\mathbf{F}^{\prime} \mathbf{F}=\mathbb{I}_{r}$, minimizar (3.5) é equivalente a maximizar $\operatorname{tr}\left[\mathbf{F}^{\prime}\left(\mathbf{Y} \mathbf{Y}^{\prime}\right) \mathbf{F}\right]$, em que $\mathbf{Y}=\left(\mathbf{Y}_{1}, \ldots, \mathbf{Y}_{T}\right)^{\prime}$. O estimador resultante $\tilde{\mathbf{F}}$ é dado por $\sqrt{T}$ vezes os autovetores correspondentes aos maiores $r$ autovalores da matriz $\mathbf{Y} \mathbf{Y}^{\prime}$ de ordem $T \times T$. Sabe-se que a solução não é única, no sentido que qualquer rotação ortogonal de $\tilde{\mathbf{F}}$ é também uma solução. Bai e Ng (2008) apresentaram um tratamento extensivo do Estimador dos CP. 
Os CP são empregados para estimar os fatores comuns, porque são simples de calcular e por suas propriedades estatísticas neste caso. Para um tratamento similar no estudo dos CP considerando outro tipo de dinâmica nas cargas, ver por exemplo, Mikkelsen et al. (2016) com um modelo VAR ou Breitung e Eickmeier (2011) com rupturas estruturais.

O seguinte resultado indica que é possível estimar consistentemente uma rotação dos fatores através dos CP mesmo com a suposição de cargas variando no tempo, considerando as suposições S1-S5. O seguinte teorema é uma versão modificada do Teorema 1 em Bates et al. (2013), adaptado às suposições deste modelo.

Teorema 3.1.1. Sob as suposições S1-S5 existe uma matriz $H$ de ordem $r \times r$ tal que

$$
T^{-1} \sum_{t=1}^{T}\left\|\tilde{\boldsymbol{F}}_{t}-H^{\prime} \boldsymbol{F}_{t}\right\|^{2}=O_{p}\left(R_{N T}\right)
$$

quando $N, T \rightarrow \infty$, em que $R_{N T}=\max \left\{\frac{1}{N}, \frac{1}{N T}, \frac{K_{1 N T}}{N^{2}}, \frac{K_{2 N T}}{N^{2} T^{2}}, \frac{K_{3 N T}}{N^{2} T^{2}}\right\}$. A matriz $H r \times r$ é definida $H=$ $\left(\boldsymbol{\Lambda}_{0}^{\prime} \boldsymbol{\Lambda}_{0} / N\right)\left(\boldsymbol{F}^{\prime} \tilde{\boldsymbol{F}} / T\right) V_{N T}^{-1}$, enquanto $K_{1 N T}, K_{2 N T}$ e $K_{3 N T}$ são definidas em $(S 4)$ e $V_{N T}$ matriz diagonal dos $r$ autovalores da matriz $(N T)^{-1} \boldsymbol{Y}^{\prime}$ em ordem decrescente.

Prova. Ver apêndice.

O Teorema 3.1.1 indica que o desvio quadrático médio entre os CP e uma rotação dos fatores comuns verdadeiros diminui quando $T, N \rightarrow \infty$. A taxa de convergência $R_{N T}$ é similar ao apresentado em Bai e $\mathrm{Ng}$ (2002). Notemos que de (3.6), os fatores comuns são identificados através de uma rotação, portanto os CP convergem para uma rotação dos verdadeiros fatores comuns $H^{\prime} \mathbf{F}_{t}$.

O seguinte resultado apresenta a relação entre a matriz de rotação existente no Teorema 3.1.1 e a matriz de covariância dos fatores quando os verdadeiros fatores são ortogonais.

Proposição 3.1.1. Sob as suposições S1-S5 e assumindo que as matrizes $\Gamma_{F}=\mathbb{I}_{r}$ e D é uma matriz diagonal com entradas distintas, então

$$
H=\mathbb{I}_{r}+O_{p}\left(C_{N T}^{-2}\right)
$$

em que $C_{N T}=\min \{\sqrt{N}, \sqrt{T}\}$.

Prova. Ver apêndice.

Este último resultado estabelece que sob certas condições a matriz de rotação dada no Teorema 3.1.1 é assintoticamente igual à matriz identidade, ou seja, que o estimador dos CP estima com maior precisão o espaço gerado pelos fatores verdadeiros para grandes valores de $N$ e $T$.

\subsection{Identificabilidade}

É bem conhecido que sem restrições de identificabilidade, os fatores e as cargas fatoriais não são separadamente identificáveis em (3.1) e (3.2) ante rotações. Notemos que para $H$ matriz de $r \times r$ invertível, 
o modelo $\mathbf{Y}_{t}=\left[\boldsymbol{\Lambda}_{0}+\boldsymbol{\Lambda}(u)\right] \mathbf{F}_{t}+\mathbf{e}_{t}$, é observasionalmente equivalente a

$$
\mathbf{Y}_{t}=\left[\boldsymbol{\Lambda}_{0}+\boldsymbol{\Lambda}(u)\right] H^{\prime-1} H^{\prime} \mathbf{F}_{t}+\mathbf{e}_{t}
$$

O Teorema 3.1.1 afirma que o estimador de componentes principais $\tilde{\mathbf{F}}_{t}$ é um estimador consistente para uma rotação dos verdadeiros fatores $H^{\prime} \mathbf{F}_{t}$. Assim para as cargas variando no tempo, o objetivo real é estimar suas rotações, isto é, $\lambda_{i}^{*^{\prime}}(u)=H^{\prime}-1 \lambda_{i}^{\prime}(u)$, para $i=1, \ldots, N$, em que $\lambda_{i}(u)$ é a $i$-ésima linha da matriz de cargas $\left[\boldsymbol{\Lambda}_{0}+\boldsymbol{\Lambda}(u)\right]$. Consequentemente com esta metodologia obtemos uma estimativa de uma rotação das cargas fatoriais verdadeiras.

Em particular, se as matrizes $\Gamma_{F}$ e $D$ são diagonais, seguindo Mikkelsen et al. (2016), a matriz de rotação $H$ converge para $\Gamma_{F}^{-1 / 2}$. Portanto, o que estimamos neste caso, é $\lambda_{i}^{*^{\prime}}(u)=\Gamma_{F}^{\prime-1 / 2} \lambda_{i}^{\prime}(u)$ para grandes valores de $N$ e $T$.

Neste trabalho assumimos a matriz de covariâncias dos fatores $\Gamma_{F}$ diagonal e não empregamos restrições na matriz $\left[\Lambda_{0}+\boldsymbol{\Lambda}(u)\right]$, tais como ortogonal, triangular inferior, entre outras, comumente requeridos nos trabalhos apresentados na literatura. Essas restrições podem não ser realistas, um vez que não são conhecidas as influências funcionais dos fatores sobre as séries observadas.

\subsection{Estimação das cargas funcionais}

Para apresentar o procedimento de estimação das cargas funcionais, suponha que temos o processo (3.1), gerado por $r$ fatores comuns $(r<N)$, considerando as suposições 3.1.1. Daqui em diante escrevemos a matriz de cargas $\left[\boldsymbol{\Lambda}(u)+\boldsymbol{\Lambda}_{0}\right]$ como uma única função no tempo $\boldsymbol{\Lambda}(u)$, dado por

$$
\mathbf{Y}_{t}=\boldsymbol{\Lambda}(u) \mathbf{F}_{t}+\mathbf{e}_{t},
$$

$\operatorname{com} t=1,2, \ldots, T$ e $u=t / T \in[0,1]$, na forma matricial, temos

$$
\left[\begin{array}{c}
Y_{1 t} \\
Y_{2 t} \\
\vdots \\
Y_{r t} \\
\vdots \\
Y_{N t}
\end{array}\right]=\left[\begin{array}{cccc}
\lambda_{11}(u) & \lambda_{12}(u) & \ldots & \lambda_{1 r}(u) \\
\lambda_{21}(u) & \lambda_{22}(u) & \ldots & \lambda_{2 r}(u) \\
\vdots & \vdots & \ddots & \vdots \\
\lambda_{r 1}(u) & \lambda_{r 2}(u) & \ldots & \lambda_{r r}(u) \\
\vdots & \vdots & \ddots & \vdots \\
\lambda_{N 1}(u) & \lambda_{N 2}(u) & \ldots & \lambda_{N r}(u)
\end{array}\right]\left[\begin{array}{c}
F_{1 t} \\
F_{2 t} \\
\vdots \\
F_{r t}
\end{array}\right]+\left[\begin{array}{c}
e_{1 t} \\
e_{2 t} \\
\vdots \\
e_{r t} \\
\vdots \\
e_{N t}
\end{array}\right]
$$

em que $\mathbf{Y}_{t}$ é um vetor $N$-dimensional de séries temporais, $\boldsymbol{\Lambda}(u)$ é a matriz de cargas fatoriais variando no tempo re-escalado com $\lambda_{i j}(u) \in \mathcal{F}$, para $i=1,2, \ldots, N$ e $j=1,2, \ldots, r, \mathbf{F}_{t}$ processo de fatores latentes e $\mathbf{e}_{t}$ processo idiossincrático. 
Notemos que, de (2.8) e da suposição (S5), a estrutura da matriz de covariância do processo $\mathbf{Y}_{t}$, tem a forma

$$
\Gamma_{Y}(u)=\boldsymbol{\Lambda}(u) \Gamma_{F} \boldsymbol{\Lambda}^{\prime}(u)+\Gamma_{e}, \quad \forall u \in[0,1]
$$

ou seja, a variância dos componentes comuns são $\Gamma_{X}(u)=\boldsymbol{\Lambda}(u) \Gamma_{F} \boldsymbol{\Lambda}^{\prime}(u)$.

Para a construção dos estimadores das cargas funcionais, assumimos que o estimador dos $r$ fatores comuns são obtidos através dos $\mathrm{CP}$ da série temporal $N$ - dimensional $\mathbf{Y}_{t}, \operatorname{com} t=1, \ldots, T$. As funções de cargas fatoriais podem ser expandidas em séries de ondaletas ortogonais como em (2.24), com a seguinte representação

$$
\lambda_{m n}(u)=\alpha_{00}^{(m n)} \phi(u)+\sum_{j=0}^{\infty} \sum_{k \in I_{j}} \beta_{j k}^{(m n)} \psi_{j k}(u),
$$

com $m=1, \ldots, N, n=1, \ldots, r$ e $I_{j}=\left\{k: k=0,1, \ldots, 2^{j}-1\right\}$. Lembremos que a representação (3.11) é possível dado que $\lambda_{m n}(u) \in \mathcal{F}$. As funções $\phi$ e $\psi_{j k}$ são construídas a partir de duas funções bases, função de escala $\phi(u)$ e a ondaleta $\psi_{j k}(u)$, em que a última é definida como uma coleção infinita de versões trasladada e escaladas como foi estabelecido na seção 2.2 .

Para um nível de resolução fixo $J<T$, a aproximação $\lambda_{m n}(u)$ de (3.11) pode ser analisada da seguinte forma

$$
\lambda_{m n}(u)=\alpha_{00}^{(m n)} \phi(u)+\sum_{j=0}^{J-1} \sum_{k \in I_{j}} \beta_{j k}^{(m n)} \psi_{j k}(u)+r_{m n}(u, J),
$$

com termo residual

$$
r_{m n}(u, J)=\sum_{j \geq J} \sum_{k \in I_{j}} \beta_{j k}^{(m n)} \psi_{j k}(u) .
$$

Note que $r_{m n}(u, J)$ é o termo de erro devido ao truncamento da expansão em ondaletas numa escala $J=J(T)$. Quanto maior o valor de $J$, menor será o erro de aproximação, isto é $r_{m n}(u, J)=o\left(T^{-1}\right)$. Observe que os valores de $j, k$ variam dependendo do nível de resolução desejado na decomposição em ondaletas. Para o processo de estimação, escolhemos a máxima resolução $J$ tal que $2^{J-1} \leq \sqrt{T} \leq 2^{J}$, ver Dahlhaus (1999), para detalhes dessa escolha. Na prática, os coeficientes $\alpha_{00}^{(m n)}, \beta_{00}^{(m n)}, \beta_{10}^{(m n)}, \ldots, \beta_{J-1,2^{J}-1}^{(m n)}$ são estimados por algum método de estimação, em particular MQ generalizados, e posteriormente empregados para construir as funções de cargas.

Suponha que são observadas $N$ séries temporais $\mathbf{Y}_{t}=\left(Y_{1 t}, Y_{2 t}, \ldots, Y_{N t}\right), \operatorname{com} t=1,2, \ldots, T$, as quais são conduzidas pelo modelo

$$
\mathbf{Y}_{t}=\boldsymbol{\Lambda}(u) \tilde{\mathbf{F}}_{t}+\mathbf{e}_{t}
$$

em que os $r$ fatores comuns $\tilde{\mathbf{F}}_{t}$ são estimados através dos CP. Assim, escrevendo cada função de carga $\lambda_{m n}(u)$ como em (3.12) e substituindo na representação matricial (3.9) obtemos 


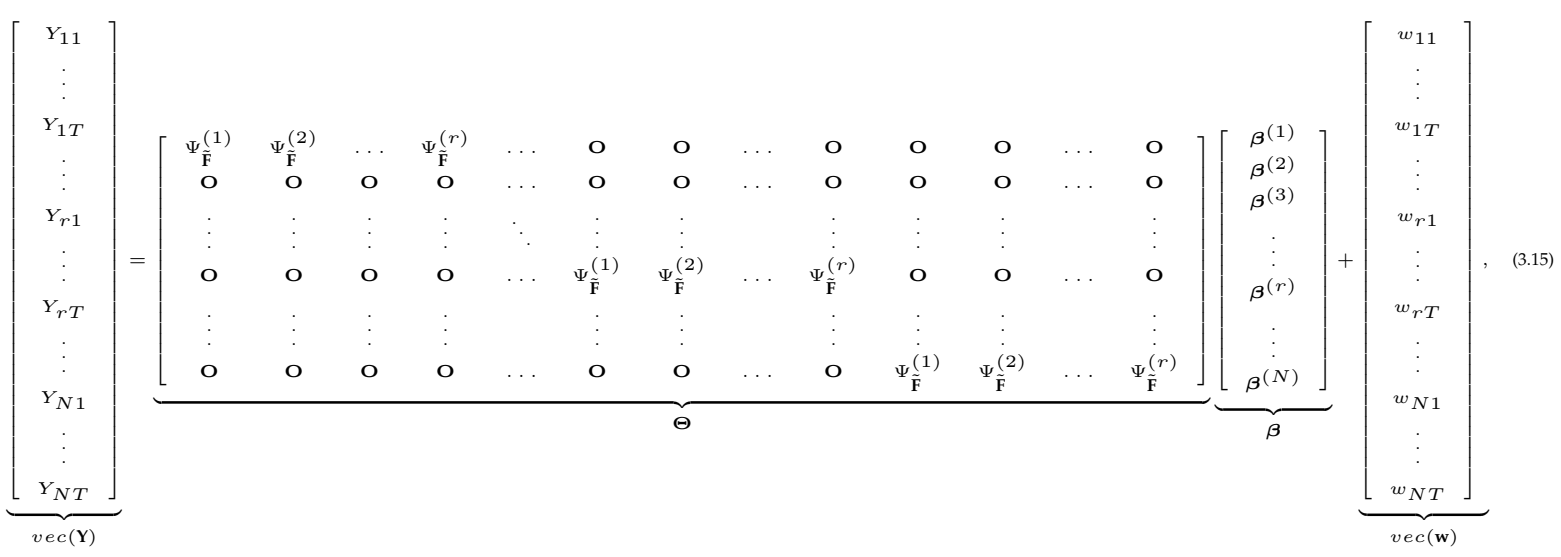

em que

$$
\Psi_{\tilde{\mathbf{F}}}^{(i)}=\left[\begin{array}{cccc}
\phi(1 / T) \tilde{F}_{i 1} & \psi_{00}(1 / T) \tilde{F}_{i 1} & \ldots & \psi_{J-1,2^{J}-1}(1 / T) \tilde{F}_{i 1} \\
\phi(2 / T) \tilde{F}_{i 2} & \psi_{00}(2 / T) \tilde{F}_{i 2} & \ldots & \psi_{J-1,2^{J}-1}(2 / T) \tilde{F}_{i 2} \\
\vdots & \vdots & \ddots & \vdots \\
\phi(T / T) \tilde{F}_{i T} & \psi_{00}(T / T) \tilde{F}_{i T} & \ldots & \psi_{J-1,2^{J}-1}(T / T) \tilde{F}_{i T}
\end{array}\right]
$$

são matrizes de ordem $T \times 2^{J}$ para $i=1,2, \ldots, r$ e $\mathbf{O}$ é a matriz nula de ordem $T \times 2^{J}$. Seja $\Psi_{r \tilde{F}}=$ $\left[\Psi_{\tilde{F}}^{(1)}, \ldots, \Psi_{\tilde{F}}^{(r)}\right]$ de ordem $T \times r 2^{J}$, portanto

$$
\Theta=\mathbb{I}_{N} \otimes \Psi_{r \tilde{F}}
$$

é uma matriz de ordem $N T \times N r 2^{J}$ que depende dos fatores estimados $\tilde{\mathbf{F}}_{t}$, das ondaletas $\psi(t)$ e o nível de resolução $J$. Os vetores parâmetros $\boldsymbol{\beta}^{(m)}=\left(\boldsymbol{\beta}^{(m 1)}, \boldsymbol{\beta}^{(m 2)}, \ldots, \boldsymbol{\beta}^{(m r)}\right)^{\prime}$ com dimensão $r 2^{J} \times 1$, com $m=$ $1,2, \ldots, N$, em que $\boldsymbol{\beta}^{(m n)}=\left(\alpha_{00}^{(m n)}, \beta_{00}^{(m n)}, \beta_{10}^{(m n)}, \ldots, \beta_{J-1,2^{J}-1}^{(m n)}\right)^{\prime}$. Notemos que cada $\boldsymbol{\beta}^{(m)}$ é composto pelos coeficientes de ondaletas das funções da $m$-ésima linha da matriz $\boldsymbol{\Lambda}(u)$. Consequentemente o comprimento do vetor parâmetro $\boldsymbol{\beta}$ do modelo (3.15) é $2^{J} N r$, o número total de coeficientes de ondaletas a serem estimados. Além disso, $w_{i t}=R_{F}^{i}(t, J)+e_{i t} \operatorname{com} i=1,2, \ldots, N$, em que $R_{F}^{i}(t, J)$ é um processo dependente dos fatores e dos erros devido ao truncamento da expansão de ondaletas da $i$-ésima linha da matriz de cargas fatoriais. O seguinte teorema fornece as estimativas lineares dos coeficientes de ondaletas das cargas fatoriais assumindo conhecida a matriz de covariâncias dos erros.

Teorema 3.3.1. Suponha que no modelo (3.15) dado por

$$
\operatorname{vec}(\boldsymbol{Y})=\mathbf{\Theta} \boldsymbol{\beta}+\operatorname{vec}(\boldsymbol{w}),
$$

as séries vec $(\mathbf{Y})$ é um vetor resposta, $\boldsymbol{\Theta}$ uma matriz de planejamento de uma regressão e assumindo que a matriz 
de variância $\Gamma_{w}$ dos erros é conhecida, então o estimador de MQ Generalizados dos Coeficientes $\boldsymbol{\beta}$ é dado por

$$
\hat{\boldsymbol{\beta}}=\left(\mathbf{\Theta}^{\prime} \Sigma_{w}^{-1} \mathbf{\Theta}\right)^{-1} \mathbf{\Theta}^{\prime} \Sigma_{w}^{-1} \operatorname{vec}(\boldsymbol{Y})
$$

em que $\Sigma_{w}$ é uma matriz de ordem $N T \times N T$ dada pelo produto Kronecker

$$
\Sigma_{w}=\Gamma_{w} \otimes \mathbb{I}_{T}=\left[\begin{array}{cccc}
\mathbb{I}_{T} \gamma_{w, 11} & \mathbb{I}_{T} \gamma_{w, 12} & \ldots & \mathbb{I}_{T} \gamma_{w, 1 N} \\
\mathbb{I}_{T} \gamma_{w, 21} & \mathbb{I}_{T} \gamma_{w, 22} & \ldots & \mathbb{I}_{T} \gamma_{w, 2 N} \\
\vdots & \vdots & \ddots & \vdots \\
\mathbb{I}_{T} \gamma_{w, N 1} & \mathbb{I}_{T} \gamma_{w, N 2} & \ldots & \mathbb{I}_{T} \gamma_{w, N N}
\end{array}\right]
$$

O teorema anterior fornece uma forma de estimar os coeficientes de ondaletas das funções de cargas fatoriais quando é assumida a existência desta aproximação. Notemos que há muitos parâmetros a serem estimados, de forma que procedimentos como estimação através de máxima verossimilhança serão computacionalmente intensivos e até mesmo não implementáveis na prática. Além disso, os erros $\mathbf{w}_{t}$ dependem dos valores de $\mathbf{Y}_{t}$ e consequentemente são autocorrelacionados, dificultando ainda mais o cálculo da verossimilhança. Por tal motivo, sugerimos um estimador baseado no método de MQ generalizados de forma a simplificar sua implementação.

Nota 3.3.1. Para cada carga $\lambda_{m n}(u)$, uma base diferente de funções de ondaletas $\phi(u)$ e $\psi_{j k}(u)$ pode ser utilizada. Com o objetivo de simplificar a ilustração, empregamos a mesma base para todas as cargas no estudo de simulação e aplicação.

A fim de ilustrar a metodologia proposta na estimação das cargas, considere o caso com $N=3$ séries temporais, e $r=2$ fatores comuns estimados como

$$
\tilde{F}_{1 t}=\sqrt{T} v_{1} \text { e } \tilde{F}_{2 t}=\sqrt{T} v_{2}
$$

em que $v_{1}$ e $v_{2}$ são os autovetores correspondentes aos dois maiores autovalores da matriz $(N T)^{-1} \mathbf{Y} \mathbf{Y}^{\prime}$, portanto

$$
\left[\begin{array}{c}
Y_{1 t} \\
Y_{2 t} \\
Y_{3 t}
\end{array}\right]=\left[\begin{array}{cc}
\lambda_{11}(u) & \lambda_{12}(u) \\
\lambda_{21}(u) & \lambda_{22}(u) \\
\lambda_{31}(u) & \lambda_{32}(u)
\end{array}\right]\left[\begin{array}{c}
\tilde{F}_{1 t} \\
\tilde{F}_{2 t}
\end{array}\right]+\left[\begin{array}{c}
e_{1 t} \\
e_{2 t} \\
e_{3 t}
\end{array}\right]
$$

para $t=1,2, \ldots, T$ e $u=t / T \in[0,1]$, o qual pode ser escrito pelas seguintes equações

$$
\begin{aligned}
Y_{1 t} & =\lambda_{11}(u) \tilde{F}_{1 t}+\lambda_{12}(u) \tilde{F}_{2 t}+e_{1 t}, \\
Y_{2 t} & =\lambda_{21}(u) \tilde{F}_{1 t}+\lambda_{22}(u) \tilde{F}_{2 t}+e_{2 t}, \\
Y_{3 t} & =\lambda_{31}(u) \tilde{F}_{1 t}+\lambda_{32}(u) \tilde{F}_{2 t}+e_{3 t} .
\end{aligned}
$$


Substituindo as funções $\lambda_{m n}(u)$, pelas respectivas decomposições em ondaletas e agrupando, obtemos

$$
\begin{aligned}
Y_{1 t} & =\alpha_{00}^{(11)}\left[\phi(u) \tilde{F}_{1 t}\right]+\sum_{j=0}^{J-1} \sum_{k \in I_{j}} \beta_{j k}^{(11)}\left[\psi_{j k}(u) \tilde{F}_{1 t}\right] \\
& +\alpha_{00}^{(12)}\left[\phi(u) \tilde{F}_{2 t}\right]+\sum_{j=0}^{J-1} \sum_{k \in I_{j}} \beta_{j, k}^{(12)}\left[\psi_{j k}(u) \tilde{F}_{2 t}\right]+w_{1 t}, \\
Y_{2 t} & =\alpha_{00}^{(21)}\left[\phi(u) \tilde{F}_{1 t}\right]+\sum_{j=0}^{J-1} \sum_{k \in I_{j}} \beta_{j k}^{(21)}\left[\psi_{j k}(u) \tilde{F}_{1 t}\right] \\
& +\alpha_{00}^{(22)}\left[\phi(u) \tilde{F}_{2 t}\right]+\sum_{j=0}^{J-1} \sum_{k \in I_{j}} \beta_{j k}^{(22)}\left[\psi_{j k}(u) \tilde{F}_{2 t}\right]+w_{2 t}, \\
Y_{3 t} & =\alpha_{00}^{(31)}\left[\phi(u) \tilde{F}_{1 t}\right]+\sum_{j=0}^{J-1} \sum_{k \in I_{j}} \beta_{j k}^{(31)}\left[\psi_{j k}(u) \tilde{F}_{1 t}\right] \\
& +\alpha_{00}^{(32)}\left[\phi(u) \tilde{F}_{2 t}\right]+\sum_{j=0}^{J-1} \sum_{k \in I_{j}} \beta_{j k}^{(32)}\left[\psi_{j k}(u) \tilde{F}_{2 t}\right]+w_{3 t},
\end{aligned}
$$

com $w_{i t}=R_{\tilde{F}}^{i}(t, J)+e_{i t}$ para $i=1,2,3$, em que $R_{\tilde{F}}^{i}(t, J)$ denota um processo dependente dos fatores estimados e dos erros devido ao truncamento da expansão de ondaletas das funções da $i$-ésima linha da matriz de cargas $\boldsymbol{\Lambda}(u)$. Os valores de $j, k$ variam dependendo do nível de resolução escolhido $J$ tal que $2^{J-1} \leq \sqrt{T} \leq 2^{J}$. Escrevendo na forma matricial as equações (3.24), obtemos

$$
\left[\begin{array}{c}
Y_{11} \\
\vdots \\
Y_{1 T} \\
Y_{21} \\
\vdots \\
Y_{2 T} \\
Y_{31} \\
\vdots \\
Y_{3 T}
\end{array}\right]=\left[\begin{array}{cccccc}
\Psi_{\tilde{F}}^{(1)} & \Psi_{\tilde{F}}^{(2)} & \mathbf{O} & \mathbf{O} & \mathbf{O} & \mathbf{O} \\
\mathbf{O} & \mathbf{O} & \Psi_{\tilde{F}}^{(1)} & \Psi_{\tilde{F}}^{(2)} & \mathbf{O} & \mathbf{O} \\
\mathbf{O} & \mathbf{O} & \mathbf{O} & \mathbf{O} & \Psi_{\tilde{F}}^{(1)} & \Psi_{\tilde{F}}^{(2)}
\end{array}\right]\left[\begin{array}{c}
\boldsymbol{\beta}^{(1)} \\
\boldsymbol{\beta}^{(2)} \\
\boldsymbol{\beta}^{(3)}
\end{array}\right]+\left[\begin{array}{c}
w_{11} \\
\vdots \\
w_{1 T} \\
w_{21} \\
\vdots \\
w_{2 T} \\
w_{31} \\
\vdots \\
w_{3 T}
\end{array}\right],
$$

em que

$$
\Psi_{\tilde{F}}^{(i)}=\left[\begin{array}{cccc}
\phi(1 / T) \tilde{F}_{i 1} & \psi_{00}(1 / T) \tilde{F}_{i 1} & \ldots & \psi_{J-1,2^{J}-1}(1 / T) \tilde{F}_{i 1} \\
\phi(2 / T) \tilde{F}_{i 2} & \psi_{00}(2 / T) \tilde{F}_{i 2} & \ldots & \psi_{J-1,2^{J}-1}(2 / T) \tilde{F}_{i 2} \\
\vdots & \vdots & \ddots & \vdots \\
\phi(T / T) \tilde{F}_{i T} & \psi_{00}(T / T) \tilde{F}_{i T} & \ldots & \psi_{J-1,2^{J}-1}(T / T) \tilde{F}_{i T}
\end{array}\right]
$$

são matrizes de ordem $T \times 2^{J}$ para $i=1,2$, $\mathbf{O}$ é a matriz nula de ordem $T \times 2^{J}$. Cada $\boldsymbol{\beta}^{(m)}=$ $\left(\boldsymbol{\beta}^{(m 1)}, \boldsymbol{\beta}^{(m 2)}\right)^{\prime}$ para $m=1,2,3$, em que $\boldsymbol{\beta}^{(m n)}=\left(\alpha_{00}^{(m n)}, \beta_{00}^{(m n)}, \ldots, \beta_{J-1,2^{J}-1}^{(m n)}\right)^{\prime}$ é o vetor de coeficientes de ondaletas de comprimento $2^{J}$, correspondente à função de carga $\lambda_{m n}(u)$. 
O modelo (3.25), pode ser escrito através de um modelo linear como segue

$$
\operatorname{vec}(\mathbf{Y})=\mathbf{\Theta} \boldsymbol{\beta}+\operatorname{vec}(\mathbf{w})
$$

onde $\operatorname{vec}(\mathbf{Y})$ e $\operatorname{vec}(\mathbf{w})$ são vetores de comprimento $(3 T), \boldsymbol{\Theta} \equiv \boldsymbol{\Theta}(\tilde{\mathbf{F}}, \psi, J)$ é uma matriz de planejamento de ordem $(3 \cdot T) \times\left(2 \cdot 3 \cdot 2^{J}\right)$ e $\boldsymbol{\beta}$ um vetor de comprimento $\left(2 \cdot 3 \cdot 2^{J}\right)$.

Portanto pelo Teorema 3.3.1 assumindo $\operatorname{vec}(\mathbf{Y})$ como o vetor de resposta, $\Theta$ a matriz de planejamento de uma regressão e assumindo a matriz de variância $\Gamma_{w}$ do processo $N$-dimensional $\mathbf{w}_{t}$ conhecida, o estimador de MQ generalizados dos coeficientes $\boldsymbol{\beta}$ é dado por

$$
\hat{\boldsymbol{\beta}}=\left(\boldsymbol{\Theta}^{\prime} \Sigma_{w}^{-1} \mathbf{\Theta}\right)^{-1} \boldsymbol{\Theta}^{\prime} \Sigma_{w}^{-1} \operatorname{vec}\left(\mathbf{Y}_{t}\right)
$$

em que $\Sigma_{w}=\Gamma_{w} \otimes \mathbb{I}_{T}$.

\subsection{Propriedades}

Com o propósito de derivar algumas propriedades do estimador de MQ dos coeficientes de ondaletas das cargas fatoriais, consideramos as seguintes suposições.

\section{S6. Ondaletas:}

a. As bases de ondaletas induzem normas no espaço dos coeficientes, as quais são análogas a normas no espaço de Besov. Consideremos um espaço de Besov $\mathcal{B}_{p, q}^{l} \operatorname{com} l \leq 1$ é um parâmetro de suavidade e $(1 \leq p, q<\infty)$ parâmetros de forma. Para o Modelo Fatorial com Cargas Funcionais, assumiremos que as cargas $\lambda(u)$ são funções reais, limitadas pertencentes ao seguinte espaço:

$$
\begin{gathered}
\mathcal{F}=\left\{\lambda(u): \lambda(u)=\alpha_{00} \phi(u)+\sum_{j=0}^{\infty} \sum_{k \in I_{j}} \beta_{j k} \psi_{j k}(u) \mid\left\|\alpha_{00}\right\|<\infty,\left\|\beta_{. .}\right\|_{l, p, q}<\infty\right\} \\
\left\|\beta_{. .}\right\|_{l, p, q}=\left[\sum_{j \geq 0}\left(2^{j s p} \sum_{k \in I_{j}}\left|\beta_{j k}\right|^{p}\right)^{q / p}\right]^{1 / q}
\end{gathered}
$$

em que $s=l+1 / 2-1 / p$ e $1<l+1 / 2-\min \{p, 2\}$.

b. As funções $\phi(u), \psi_{j k}(u) \in C^{d}[0,1]$ e têm suporte compacto. Além disso $\int \phi(u) d u=1 \mathrm{e}$ $\int \psi(u) u^{k} d u=0$ para $0 \leq k \leq d$.

c. Existe um $\gamma \geq 0$ tal que $\left|\operatorname{cum}_{n}\left(\mathbf{w}_{t}\right)\right| \leq K^{n}(n !)^{1+\gamma}, \forall n, t \in \mathbb{N}$.

d. Os componentes comuns são localmente estacionários.

e. $\left\|\Gamma_{w}^{-1}\right\|<\infty$. 
Considerando as expansões em funções de ondaletas nas cargas fatoriais do modelo fatorial proposto e com as suposições anteriores, é possível provar a normalidade assintótica do estimador linear dos coeficientes de ondaletas das cargas funcionais. Antes de apresentar o resultado principal, é enunciado um lema que será usado na prova.

Lema 3.4.1. Considerando que as suposições $(S 6 . a-S 6 . e)$ são satisfeitas

$$
T H_{T}\left[P^{\prime} P\right]^{-1} H_{T}^{\prime} \stackrel{p}{\rightarrow} \Gamma
$$

em que $\Gamma=\lim _{T \rightarrow \infty} T H_{T} \mathbb{E}\left[P^{\prime} P\right]^{-1} H_{T}^{\prime}$.

Prova. Ver Sato et al. (2007).

Teorema 3.4.1. Assumindo o modelo $\operatorname{vec}(\boldsymbol{Y})=\boldsymbol{\Theta} \boldsymbol{\beta}+\operatorname{vec}(\boldsymbol{w})$ do Terorema 3.3.1 e considerando que as condições de S6. a - S6. e estão satisfeitas e que os erros são gaussianos com matriz de covariância $\Gamma_{w}$ conhecida, então

$$
\sqrt{T} H_{T}(\hat{\boldsymbol{\beta}}-\boldsymbol{\beta}) \stackrel{a}{\rightarrow} \mathbf{Z}
$$

em que $\mathbf{Z}$ é um vetor aleatório com distribuição normal $k$-dimensional com média zero e matriz de covariância $\Gamma$ dada por

$$
\Gamma=\lim _{T \rightarrow \infty} T H_{T} \mathbb{E}\left(P^{\prime} P\right)^{-1} H_{T}^{\prime}
$$

A matriz $H_{T}$ é utilizada para formalizar a convergência em distribuição e para obter subpartições finita dos parâmetros. A matriz Pédefinida como $P=\Sigma_{w}^{-1 / 2} \boldsymbol{\Theta}$.

Prova. Ver apêndice.

\subsection{Algoritmo}

Aqui apresentamos os passos para realizar a estimação dos parâmetros do modelo (3.1), empregando os CP e o Teorema 3.3.1.

Passo 1. Utilizamos os Componentes Principales CP para estimar $\mathbf{F}_{t}$. Esse estimador é dado por

$$
\tilde{\mathbf{F}}=\sqrt{T}\left[v_{1}, v_{2}, \ldots, v_{r}\right]
$$

em que cada $v_{i}$ é o autovetor correspondente ao autovalor $\lambda_{i}$, para $i=1, \ldots, r$, na ordem decrescente da matriz $(N T)^{-1} \mathbf{Y} \mathbf{Y}^{\prime}$. Aquí $\mathbf{Y}$ e $\tilde{\mathbf{F}}$ denotam matrizes de ordem $T \times N$ e $T \times r$, respectivamente. Em seguida, as funções de carga da matriz $\boldsymbol{\Lambda}(t)$ são expandidas em séries de ondaletas ortogonais

$$
\lambda_{m n}(t)=\alpha_{00}^{(m n)} \phi(t)+\sum_{j=0}^{J-1} \sum_{k \in I_{j}} \beta_{j k}^{(m n)} \psi_{j k}(t),
$$


com $I_{j}=\left\{k: k=0,1, \ldots, 2^{j}-1\right\}, m=1, \ldots, N$ e $n=1, \ldots, r$. Escrevemos as equações (3.14) como um modelo linear

$$
\operatorname{vec}(\mathbf{Y})=\boldsymbol{\Theta}[\tilde{\mathbf{F}}, \psi, \phi] \boldsymbol{\beta}+\operatorname{vec}(\mathbf{w})
$$

onde $\operatorname{vec}(\mathbf{Y})$ e $\operatorname{vec}(\mathbf{w})$ são vetores de dimensão $N T \times 1$, e as dimensões de $\boldsymbol{\Theta}[\tilde{\mathbf{F}}, \psi, \phi]$ e $\boldsymbol{\beta}$ são $\left(N T \times 2^{J} N r\right)$ e $\left(2^{J} N r \times 1\right)$, respetivamente, em que $J$ indica o nível de resolução escolhido nas expansões de ondaletas.

Passo 2. Denotando $\boldsymbol{\Theta}[\tilde{\mathbf{F}}, \psi, \phi] \equiv \boldsymbol{\Theta}, \mathrm{e} \mathbf{Z}=\operatorname{vec}(\mathbf{Y})$ obtemos um estimador de MQ para os coeficientes de ondaletas dado por

$$
\hat{\boldsymbol{\beta}}=\left(\boldsymbol{\Theta}^{\prime} \Sigma_{w}^{-1} \boldsymbol{\Theta}\right)^{-1} \boldsymbol{\Theta}^{\prime} \Sigma_{w}^{-1} \mathbf{Z}
$$

onde empregamos como chute inicial a matriz $\Sigma_{w}=\mathbb{I}_{N T}$.

Paso 3. Usamos os coeficientes estimados no passo 2 e construímos as cargas $\hat{\Lambda}(t)^{(0)}=\left\{\hat{\lambda}_{m n}^{(0)}(t)\right\}_{m=1, \ldots, N^{\prime}}^{n=1, \ldots, r}$ em que

$$
\hat{\lambda}_{m n}^{(0)}(t)=\hat{\alpha}_{00}^{(m n)} \phi(t)+\sum_{j=0}^{J-1} \sum_{k \in I_{j}} \hat{\beta}_{j k}^{(m n)} \psi_{j k}(t) .
$$

Paso 4. Com $\hat{\boldsymbol{\Lambda}}(t)^{(0)}$ obtemos os residuais $\mathbf{Y}_{t}-\hat{\boldsymbol{\Lambda}}(t)^{(0)} \tilde{\mathbf{F}}_{t}=\hat{e}_{t}^{(0)}$ e calculamos $\hat{\Gamma}_{e}^{(0)}=\sum_{t=1}^{T} \hat{e}_{t}^{(0)} \hat{e}_{t}^{(0)^{\prime}} / T$.

Paso 5. Voltamos ao paso 2 com $\Sigma_{w}=\hat{\Gamma}_{e}^{(0)}$. Reiteramos o proceso para obter uma sucessão de matrizes $\left\{\hat{\boldsymbol{\Lambda}}(t)^{(i)}, \hat{\Gamma}_{e}^{(i)}\right\}_{i=1, \ldots, n}$, para $n$ iterações. Paramos o processo quando

$$
\left\|\hat{\boldsymbol{\Lambda}}(t)^{(i-1)}-\hat{\boldsymbol{\Lambda}}(t)^{(i)}\right\|<\delta
$$

para algum $\delta>0$, em que $\|\cdot\|$ denota a norma de Frobenius, para $t=1, \ldots, T$. A estimativa para a matriz de erros idiossincráticos é escolhida na $n$-ésima iteração, $\hat{\Gamma}_{e}^{(n)}$.

Ao fim de entender o procedimento para sua implementação computacional, descrevemos o processo através do algoritmo a seguir.

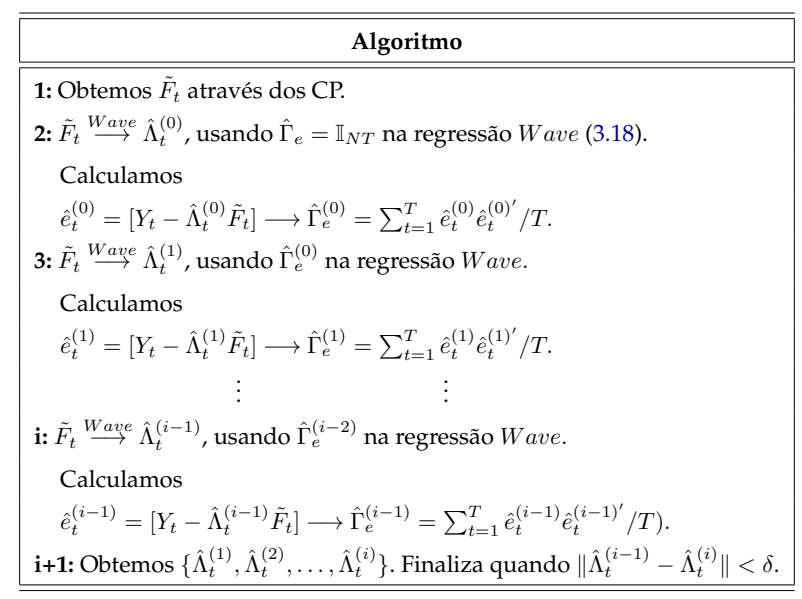




\section{Capítulo 4}

\section{Simulações}

Neste capítulo apresentamos um estudo de simulação para avaliar o desempenho da metodologia proposta de estimação em dois estágios.

\subsection{Esquema de simulação}

Os processos pelos quais vamos gerar as séries multivariadas são

$$
\begin{aligned}
Y_{i t} & =\boldsymbol{\lambda}_{i}^{\prime}(t) \mathbf{F}_{t}+e_{i t}, \quad i=1, \ldots, N \text { e } t=1, \ldots, T \\
F_{k t}\left(1-\theta_{k} B\right) & =\eta_{k t}, \quad k=1, \ldots, r . \boldsymbol{\eta}_{t} \sim \mathcal{N}_{r}\left(0, \operatorname{diag}\left\{1-\theta_{1}^{2}, \ldots, 1-\theta_{r}^{2}\right\}\right), \\
\mathbf{e}_{t} & \sim \mathcal{N}_{N}\left(0, \Gamma_{e}\right),
\end{aligned}
$$

em que consideramos a matriz $\Gamma_{e}$ com as seguintes estruturas:

$$
\begin{aligned}
& \Gamma_{e}=\text { Toep }=\left\{\gamma^{|i-j|}\right\}_{i, j=1, \ldots, N} \\
& \Gamma_{e}=\text { Diag, matriz diagonal. }
\end{aligned}
$$

- $Y_{i t}: i$-ésima série temporal no instante $t$,

- $\boldsymbol{\lambda}_{i}^{\prime}(t)=\left(\lambda_{i 1}(t), \ldots, \lambda_{i r}(t)\right)$ : vetor de cargas fatoriais de dimensão $1 \times r$ no instante $t$

- $\mathbf{F}_{t}=\left(F_{1 t}, \ldots, F_{r t}\right)^{\prime}:$ vetor de fatores de dimensão $r \times 1$ no tempo $t$,

- $\boldsymbol{\eta}_{t}=\left(\eta_{1 t}, \ldots, \eta_{r t}\right)^{\prime}:$ vetor de erros $r \times 1$, no tempo $t$,

- $\mathbf{e}_{t}=\left(e_{1 t}, \ldots, e_{N t}\right)^{\prime}:$ vetor de $N \times 1$, no instante $t$. 
Neste estudo, utilizamos $\Gamma_{e}=$ Toep, $\operatorname{com} \gamma=0,7$, o qual garante que o processo idiossincrático seja correlacionado, mas sem dependência temporal. Para $\Gamma_{e}=\operatorname{Diag}$ empregamos as matrizes $\Gamma_{e}=$ $\operatorname{diag}\{0.8,1.9, \operatorname{rep}(1,6), 0.7,1.1,0.9,1.3, \operatorname{rep}(1,8)\}$ para $N=20$ e $\Gamma_{e}=\operatorname{diag}\{0.8,1.9, \operatorname{rep}(1,6), 0.7,1.1,0.9$, $1.3, \operatorname{rep}(1,8), 1.2,0.9,1.5,0.7,1.6,1.3,1.7,0.8,1.2,1.5\}$ para $N=30$. A matriz de cargas funcionais $\boldsymbol{\Lambda}(t)$ de $N \times r$ é composta de diferentes funções suaves definidas a seguir:

$$
\begin{aligned}
& \lambda_{1}(u)=(10 / 7) \sqrt{u}, \\
& \lambda_{2}(u)=(1 / 2) \cos (1,1 \pi u), \\
& \lambda_{3}(u)=(6 / 10) u-1, \\
& \lambda_{4}(u)=(6 / 10) u^{2}, \\
& \lambda_{5}(u)=(1 / 2) \sin (1,5 \pi u), \\
& \lambda_{6}(u)=(-2 / 5) \cos (\pi u), \\
& \lambda_{7}(u)=(-7 / 10) \cos (0,6 \pi u), \\
& \lambda_{8}(u)=(1 / 2) \cos (\pi u), \\
& \lambda_{9}(u)=(-1 / 2) \sin (1,2 \pi u), \\
& \lambda_{10}(u)=(1 / 2) \sin (\pi u) \text {, } \\
& \lambda_{11}(u)=(-3 / 5) \cos (\pi u), \\
& \lambda_{12}(u)=(2 / 5) \cos (-3 \pi u), \\
& \lambda_{13}(u)=(-7 / 10) \sqrt{u}, \\
& \lambda_{14}(u)=(3 / 5) \sqrt{1,2 u}, \\
& \lambda_{15}(u)=(1 / 2) \exp (0,8 u), \\
& \lambda_{16}(u)=(1 / 2) \log (0,2+1,2 u), \\
& \lambda_{17}(u)=(-1 / 2) \log (0,3+u), \\
& \lambda_{18}(u)=(2 / 5) \cos (1,1 \pi u), \\
& \lambda_{19}(u)=(-2 / 5) \cos (1,1 \pi u) \text {, } \\
& \lambda_{20}(u)=(2 / 5) \cos (0,8 \pi u), \\
& \lambda_{21}(u)=\lambda_{1}+\lambda_{2}, \\
& \lambda_{22}(u)=\lambda_{1}+\lambda_{3}, \\
& \lambda_{23}(u)=0,5\left(\lambda_{1}+\lambda_{4}\right), \\
& \lambda_{24}(u)=0,7\left(\lambda_{1}+\lambda_{5}\right), \\
& \lambda_{25}(u)=0,5\left(\lambda_{1}+\lambda_{6}\right), \\
& \lambda_{26}(u)=1,5\left(\lambda_{2}+\lambda_{7}\right), \\
& \lambda_{27}(u)=\lambda_{1}+\lambda_{1}, \\
& \lambda_{28}(u)=0,6\left(\lambda_{1}+\lambda_{9}\right), \\
& \lambda_{29}(u)=0,5\left(\lambda_{1}+\lambda_{10}\right), \\
& \lambda_{30}(u)=0,3\left(\lambda_{1}+\lambda_{11}\right),
\end{aligned}
$$

$$
\begin{aligned}
& \lambda_{31}(u)=\lambda_{1}+\lambda_{12}, \\
& \lambda_{32}(u)=0,7\left(\lambda_{2}+\lambda_{13}\right), \\
& \lambda_{33}(u)=0,3\left(\lambda_{1}+\lambda_{14}\right), \\
& \lambda_{34}(u)=0,4\left(\lambda_{1}+\lambda_{15}\right), \\
& \lambda_{35}(u)=0,4\left(\lambda_{1}+\lambda_{16}\right), \\
& \lambda_{36}(u)=5\left(\lambda_{1}+\lambda_{17}\right), \\
& \lambda_{37}(u)=\lambda_{1}+\lambda_{18}, \\
& \lambda_{38}(u)=0,4\left(\lambda_{1}+\lambda_{19}\right), \\
& \lambda_{39}(u)=2\left(\lambda_{1}+\lambda_{20}\right), \\
& \lambda_{40}(u)=2\left(\lambda_{1}+\lambda_{2}+\lambda_{3}\right), \\
& \lambda_{41}(u)=\lambda_{1}+\lambda_{2}, \\
& \lambda_{42}(u)=\lambda_{2}+\lambda_{13}, \\
& \lambda_{43}(u)=0,4\left(\lambda_{3}+\lambda_{4}\right), \\
& \lambda_{44}(u)=0,6\left(\lambda_{4}+\lambda_{15}\right), \\
& \lambda_{45}(u)=1,5\left(\lambda_{5}+\lambda_{6}\right), \\
& \lambda_{46}(u)=0,6\left(\lambda_{6}+\lambda_{17}\right), \\
& \lambda_{47}(u)=0,5\left(\lambda_{7}+\lambda_{10}\right), \\
& \lambda_{48}(u)=0,6\left(\lambda_{8}+\lambda_{19}\right), \\
& \lambda_{49}(u)=0,9\left(\lambda_{9}+\lambda_{1}\right), \\
& \lambda_{50}(u)=0,2\left(\lambda_{10}+\lambda_{1}\right), \\
& \lambda_{51}(u)=\lambda_{11}+\lambda_{2}, \\
& \lambda_{52}(u)=0,4\left(\lambda_{12}+\lambda_{17}\right), \\
& \lambda_{53}(u)=0,6\left(\lambda_{13}+\lambda_{4}\right), \\
& \lambda_{54}(u)=0,3\left(\lambda_{14}+\lambda_{13}\right), \\
& \lambda_{55}(u)=0,6\left(\lambda_{15}+\lambda_{11}\right) \text {, } \\
& \lambda_{56}(u)=4\left(\lambda_{16}+\lambda_{20}\right), \\
& \lambda_{57}(u)=\lambda_{17}+\lambda_{18}, \\
& \lambda_{58}(u)=0,7\left(\lambda_{18}+\lambda_{9}\right), \\
& \lambda_{59}(u)=1,7\left(\lambda_{19}+\lambda_{20}\right) \text {, } \\
& \lambda_{60}(u)=2,1\left(\lambda_{20}+\lambda_{13}\right) .
\end{aligned}
$$

Nas Figuras 4.1 e 4.2 apresentamos os gráficos de cada função de carga empregada no estudo de simulação. 

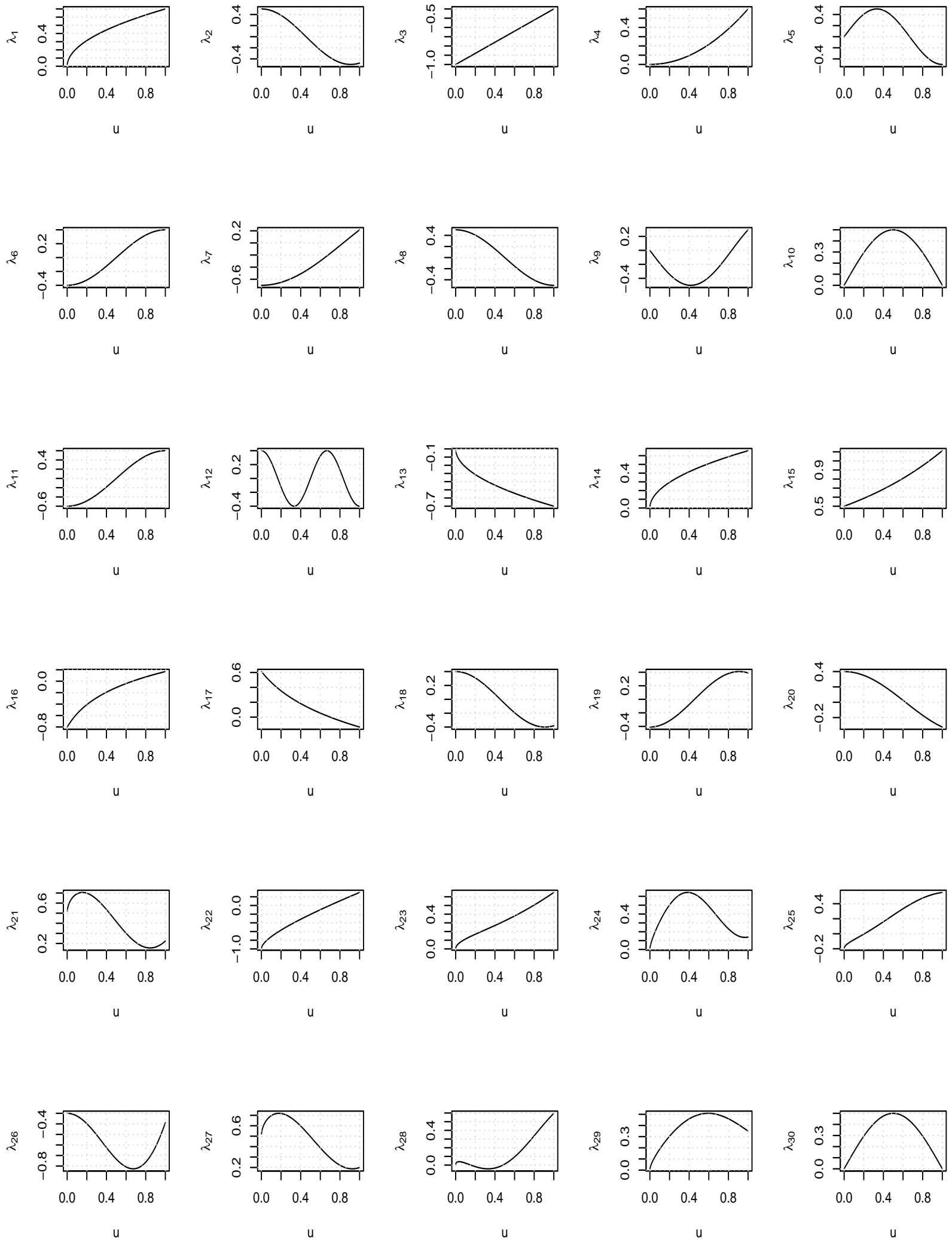

Figura 4.1: Funções utilizadas como cargas fatoriais. 


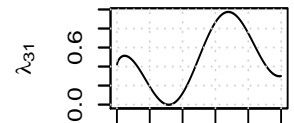

$\begin{array}{lll}0.0 & 0.4 & 0.8\end{array}$

u
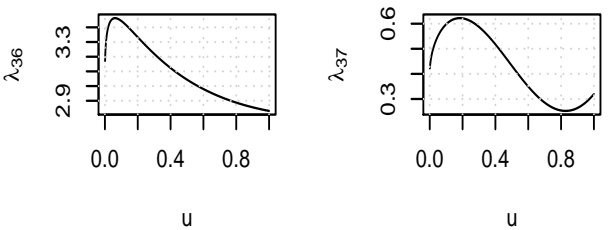

u

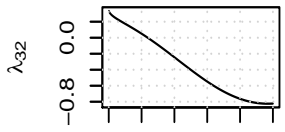

$\begin{array}{lll}0.0 & 0.4 & 0.8\end{array}$
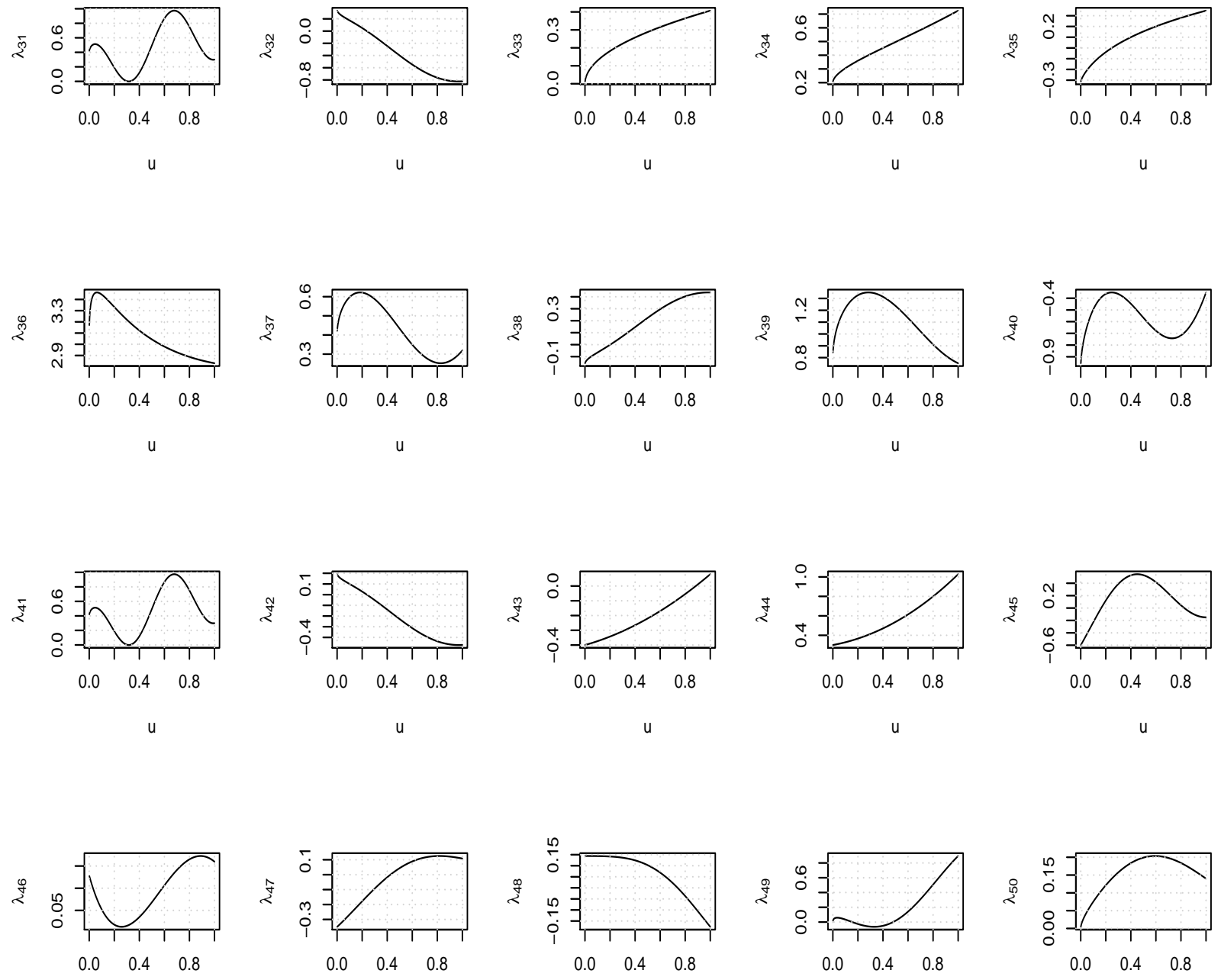

u

u

u

u

u
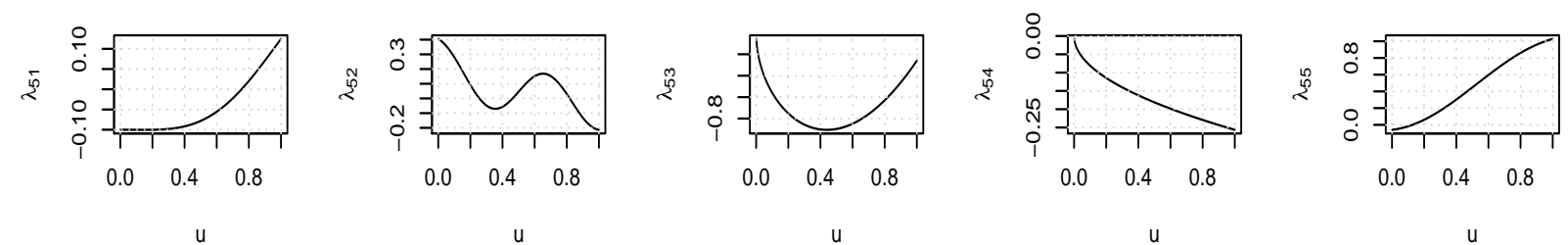

u

u
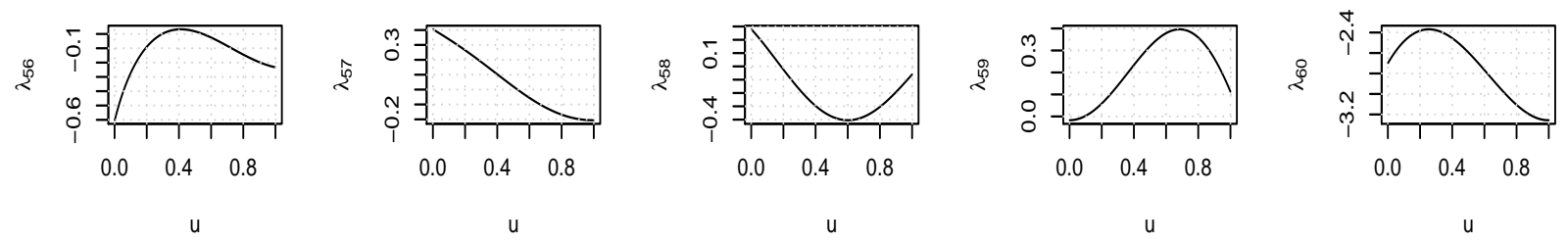

Figura 4.2: Funções utilizadas como cargas fatoriais. 
Neste estudo de simulação foram simulados os seguintes casos:

a. Modelo com um fator $(r=1), N=20, T \in\{512,1024,2048\}, \Gamma_{e}=$ Diag e $\theta=0$.

b. Modelo com um fator $(r=1), N=20, T \in\{512,1024,2048\}, \Gamma_{e}=$ Diag e $\theta=0.5$.

c. Modelo com um fator $(r=1), N=20, T \in\{512,1024,2048\}, \Gamma_{e}=$ Toep e $\theta=0$.

d. Modelo com um fator $(r=1), N=20, T \in\{512,1024,2048\}, \Gamma_{e}=$ Toep e $\theta=0.5$.

e. Modelo com um fator $(r=1), N=30, T \in\{512,1024,2048\}, \Gamma_{e}=$ Diag e $\theta=0$.

$f$. Modelo com um fator $(r=1), N=30, T \in\{512,1024,2048\}, \Gamma_{e}=$ Diag e $\theta=0.5$.

g. Modelo com um fator $(r=1), N=30, T \in\{512,1024,2048\}, \Gamma_{e}=$ Toep e $\theta=0$.

h. Modelo com um fator $(r=1), N=30, T \in\{512,1024,2048\}, \Gamma_{e}=$ Toep e $\theta=0.5$.

i. Modelo com dois fatores $(r=2), N=20, T \in\{512,1024,2048\}, \Gamma_{e}=$ Diag e $\theta=0$.

$j$. Modelo com dois fatores $(r=2), N=20, T \in\{512,1024,2048\}, \Gamma_{e}=$ Diag e $\theta=0.5$.

$k$. Modelo com dois fatores $(r=2), N=20, T \in\{512,1024,2048\}, \Gamma_{e}=$ Toep e $\theta=0$.

l. Modelo com dois fatores $(r=2), N=20, T \in\{512,1024,2048\}, \Gamma_{e}=$ Toep e $\theta=0.5$.

m. Modelo com dois fatores $(r=2), N=30, T \in\{512,1024,2048\}, \Gamma_{e}=$ Diag e $\theta=0$.

$n$. Modelo com dois fatores $(r=2), N=30, T \in\{512,1024,2048\}, \Gamma_{e}=$ Diag e $\theta=0.5$.

o. Modelo com dois fatores $(r=2), N=30, T \in\{512,1024,2048\}, \Gamma_{e}=$ Toep e $\theta=0$.

p. Modelo com dois fatores $(r=2), N=30, T \in\{512,1024,2048\}, \Gamma_{e}=$ Toep e $\theta=0.5$.

As cargas foram empregadas da seguinte forma:

- Nos casos $(a)-(d)$, utilizamos as cargas $\lambda_{1}, \ldots, \lambda_{20}$ para o primeiro fator.

- Nos casos $(e)-(h)$, utilizamos as cargas $\lambda_{1}, \ldots, \lambda_{30}$ para o primeiro fator.

- Nos casos $(i)-(l)$, utilizamos as cargas $\lambda_{1}, \ldots, \lambda_{20}$ para o primeiro fator e $\lambda_{21}, \ldots, \lambda_{40}$ para o segundo fator.

- Nos casos $(m)-(p)$, utilizamos as cargas $\lambda_{1}, \ldots, \lambda_{30}$ para o primeiro fator e $\lambda_{31}, \ldots, \lambda_{60}$ para o segundo fator. 
Para cada caso, foram geradas 1000 réplicas. Desconsideramos as 100 primeiras observações geradas em cada réplica. Os dados simulados são padronizados antes de extrair os CP. Com o objetivo de comparar os estimadores obtidos pela metodologia proposta, com os processos geradores de dados, rotamos os CP para comparar com os fatores verdadeiros. A rotação ótima $A^{*}$ de $r \times r$ é obtida maximizando $\operatorname{tr}[\operatorname{corr}(\mathbf{F}, \tilde{\mathbf{F}} A)] .{ }^{1}$ Tanto os fatores estimados quanto simulados são filtrados e re-escalados para ter o mesmo desvio padrão fazendo

$$
\tilde{F}_{k}^{*}=\frac{\sigma\left(F_{k}\right)}{\sigma\left(\tilde{F}_{k}\right)} \tilde{F}_{k}, k=1, \ldots, r
$$

em que $\tilde{F}_{k}$ é a $k$-esima coluna da matriz dos CP rotadas $\tilde{\mathbf{F}} A^{*}$. Esses $\mathrm{CP}$ principais são tratados como observados, e empregados no modelo de regressão $\operatorname{vec}(\mathbf{Y})=\boldsymbol{\Theta}\left[\tilde{\mathbf{F}}^{*}, \psi, \phi\right] \boldsymbol{\beta}+\operatorname{vec}(\mathbf{w})$ para estimar os coeficientes de ondaletas $\boldsymbol{\beta}$, em que $\tilde{\mathbf{F}}^{*}=\left(\tilde{F}_{1}^{*}, \ldots, \tilde{F}_{r}^{*}\right)$.

O desempenho do estimador dos fatores comuns $\tilde{\mathbf{F}}$ é medido pela estatística

$$
R_{\tilde{F}, F}^{2}=\frac{\operatorname{tr}\left[\mathbf{F}^{\prime} \tilde{\mathbf{F}}\left(\tilde{\mathbf{F}}^{\prime} \tilde{\mathbf{F}}\right)^{-1} \tilde{\mathbf{F}}^{\prime} \mathbf{F}\right]}{\operatorname{tr}\left[\mathbf{F}^{\prime} \mathbf{F}\right]}
$$

em que $\tilde{\mathbf{F}}$ é matriz $T \times r$ de fatores estimados (4.6) e $\mathbf{F}$ de $T \times r$ de fatores simulados. Usamos a estatística $R_{\tilde{F}, F}^{2}$ como Bates et al. (2013), a qual é um $R^{2}$ multivariado da regressão dos fatores verdadeiros sobre os CP. É menor que 1, e tende para 1 quando a correlação conônica entre os fatores e os CP tende para 1.

O desempenho do estimador das cargas fatoriais é medido usando o EQM diferença entre cargas estimadas e simuladas, isto é, para $j=1, \ldots, 1000$, definimos

$$
\operatorname{EQM}(j)=(N T)^{-1} \sum_{t=1}^{T}\left\|\hat{\boldsymbol{\Lambda}}^{(j)}(t)-\mathbf{\Lambda}(t)\right\| .
$$

É escolhido como estimador da matriz de cargas fatoriais $\boldsymbol{\Lambda}(t)$, aquela trajetória tal que

$$
\hat{\mathbf{\Lambda}}(t)=\left\{\hat{\boldsymbol{\Lambda}}^{(m)}(t): E Q M_{m}=\text { mediana }\{E Q M(1), \ldots, E Q M(1000)\}\right\}
$$

Na Tabela 4.1, $E Q M_{m}$ indica o EQM da matriz de cargas escolhida segundo (4.9), enquanto que $R_{\widetilde{F}, F}^{2}$ especifica o desempenho dos $\mathrm{CP}$ correspondentes.

As linhas tracejadas nas cargas das Figuras 4.3 - 4.18 indicam um intervalo de confiança bootstrap com um erro padrão. Essas bandas foram geradas realizando $B=100$ réplicas remostrando os erros

$$
\hat{\mathbf{e}}_{t}=\mathbf{Y}_{t}-\hat{\boldsymbol{\Lambda}}(t) \tilde{\mathbf{F}}_{t}
$$

Para gerar cada série temporal multivariada, utilizamos o software $r$ - project a função $\operatorname{mvrnorm}(\cdot)$

\footnotetext{
${ }^{1}$ A solução é dada por $A^{*}=V U$ em que $V$ e $U$ são matrizes ortogonais da decompisição $\operatorname{corr}(F, \tilde{F})=U S V^{\prime}$. Quando o número de componentes principais $k$ não é igual ao número de fatores $r$, rotamos os primeiros $l=\min \{k, r\} \mathrm{CP}$. Eickmeir et al. (2015) usa a mesma rotação.
} 
do pacote $M A S S$ para gerar os erros dos fatores e os erros idiossincráticos, também os pacotes wavelets e waveslim do mesmo software foram utilizados para obter as ondaletas Haar e D8.

\subsection{Resultados}

Na Tabela 4.1 são apresentamos os resultados obtidos em cada um dos casos, em que especificamos diferentes combinações de $N, T, r$, ondaletas, matriz de erros idiossincráticos $\Gamma_{e}$ e dependência temporal dos fatores através de $\theta$.

\begin{tabular}{|c|c|c|c|c|c|c|c|c|c|c|}
\hline \multicolumn{2}{|c|}{ Ondaleta } & \multicolumn{4}{|c|}{ Haar } & \multicolumn{4}{|c|}{ D8 } & \multirow[b]{2}{*}{$\Gamma_{e}$} \\
\hline$\overline{N N}$ & $\bar{T}$ & $R_{\tilde{F}, F}^{2}$ & $E Q M_{m}$ & $R_{\tilde{F}, F}^{2}$ & $E Q M_{m}$ & $R_{\tilde{\tilde{F}, F}}^{2}$ & $E Q M_{m}$ & $R_{\tilde{F}, F}^{2}$ & $\overline{E Q M_{m}}$ & \\
\hline \multicolumn{2}{|c|}{ Fator } & \multicolumn{2}{|c|}{$\theta=0$} & \multicolumn{2}{|c|}{$\theta=0,5$} & \multicolumn{2}{|c|}{$\theta=0$} & \multicolumn{2}{|c|}{$\theta=0,5$} & \\
\hline \multicolumn{11}{|c|}{$r=1$} \\
\hline \multirow{3}{*}{20} & 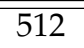 & 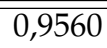 & 0,0035 & 0,9230 & 0,0037 & $\bar{~} 0,9763$ & 0,00048 & 0,9324 & 0,00057 & \multirow{3}{*}{ Diag } \\
\hline & 1024 & 0,9612 & 0,0016 * & 0,9311 & 0,0020 * & 0,9822 & 0,00021 * & 0,9023 & $0^{0,00036}{ }^{\star}$ & \\
\hline & 2048 & 0,9673 & 0,0005 & 0,9320 & 0,0017 & 0,9781 & 0,00010 & 0,9222 & 0,00023 & \\
\hline \multirow{3}{*}{30} & 512 & 0,9671 & 0,0026 & 0,9215 & 0,0041 & 0,9787 & 0,00032 & 0,9458 & 0,00042 & \multirow{3}{*}{ Diag } \\
\hline & 1024 & 0,9730 & 0,0011 & 0,9142 & 0,0024 & 0,9877 & 0,00019 & 0,9410 & 0,00021 & \\
\hline & 2048 & 0,9621 & 0,0004 & 0,9137 & 0,0005 & 0,9718 & 0,00008 & 0,9358 & 0,00014 & \\
\hline \multirow{3}{*}{20} & 512 & 0,9233 & 0,0247 & 0,8123 & 0,0686 & 0,9561 & 0,0069 & 0,8332 & 0,0371 & \multirow{3}{*}{ Toep } \\
\hline & 1024 & 0,9401 & 0,0122 * & 0,8256 & 0,0591 * & 0,9675 & 0,0048 & 0,8123 & 0,0202 * & \\
\hline & 2048 & 0,9363 & 0,0091 & 0,8545 & 0,0200 & 0,9732 & 0,0012 & 0,8290 & 0,0127 & \\
\hline \multirow{3}{*}{30} & 512 & 0,9234 & 0,0126 & 0,8095 & 0,0471 & 0,9476 & 0,0051 & 0,8231 & 0,0293 & \multirow{3}{*}{ Tоер } \\
\hline & 1024 & 0,9128 & 0,0098 & 0,8421 & 0,0211 & 0,9450 & 0,0033 & 0,8431 & 0,0201 & \\
\hline & 2048 & 0,9178 & 0,0032 & 0,8370 & 0,0175 & 0,9554 & 0,0006 & 0,8500 & 0,0094 & \\
\hline \multicolumn{11}{|c|}{$r=2$} \\
\hline \multirow{3}{*}{20} & 512 & 0,9341 & 0,0103 & 0,8542 & 0,197 & 0,9447 & 0,00101 & 0,8655 & 0,00232 & \multirow{3}{*}{ Diag } \\
\hline & 1024 & 0,9421 & 0,0076 * & 0,8135 & 0,090 * & 0,9347 & 0,0082 * & 0,8764 & 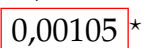 & \\
\hline & 2048 & 0,9127 & 0,0020 & 0,8236 & 0,0053 & 0,9470 & 0,00050 & 0,8455 & 0,00093 & \\
\hline \multirow{3}{*}{30} & 512 & 0,9236 & 0,0098 & 0,8456 & 0,0128 & 0,9560 & 0,0071 & 0,8521 & 0,0127 & \multirow{3}{*}{ Diag } \\
\hline & 1024 & 0,9547 & 0,0051 & 0,8125 & 0,0090 & 0,9487 & 0,0047 & 0,8671 & 0,0079 & \\
\hline & 2048 & 0,9341 & 0,0011 & 0,8268 & 0,0027 & 0,9498 & 0,0020 & 0,8601 & 0,0060 & \\
\hline \multirow{3}{*}{20} & 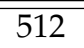 & 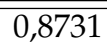 & 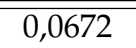 & $\overline{00,6932}$ & 0,0891 & "0,8911 & 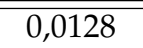 & "0,6871 & 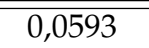 & \multirow{3}{*}{ Tоер } \\
\hline & 1024 & 0,8634 & 0,0501 * & 0,6853 & 0,0702 * & 0,9026 & 0,0100 * & 0,7001 & 0,0337 * & \\
\hline & 2048 & 0,8911 & 0,0137 & 0,7723 & 0,0433 & 0,8971 & 0,0091 & 0,7307 & 0,0108 & \\
\hline \multirow{3}{*}{30} & 512 & 0,8531 & 0,0472 & 0,7984 & 0,0621 & 0,9021 & 0,0117 & 0,7815 & 0,0311 & \multirow{3}{*}{ Tоер } \\
\hline & 1024 & 0,8671 & 0,0231 & 0,7730 & 0,0539 & 0,9126 & 0,0090 & 0,7270 & 0,0276 & \\
\hline & 2048 & 0,8451 & 0,0111 & 0,8200 & 0,0311 & 0,8732 & 0,0068 & 0,7305 & 0,0109 & \\
\hline
\end{tabular}

Tabela 4.1: As colunas $N$ e $T$ indicam os tamanhos amostrais e $r$ número de fatores. A coluna $R_{\tilde{F}, F}^{2}$ apresenta a estatística para o estimador dos CP. A coluna EQM $M_{m}$, indica a mediana do Erro Quadrático Médio entre as cargas verdadeiras e as estimadas. A coluna $\Gamma_{e}$ indica a matriz dos erros idiossincráticos, Diag matriz diagonal e Toep matriz Toeplitz de parâmetro $\gamma=0,7$. O parâmetro $\theta$ determina o grau de correlação serial dos fatores.

Os desempenhos dos estimadores das cargas funcionais $\hat{\boldsymbol{\Lambda}}(t)$ das trajetórias simuladas correspondentes ao definido em (4.9) são mostradas nas Figuras 4.11 - 4.18. Em cada figura especificamos o caso considerado. 

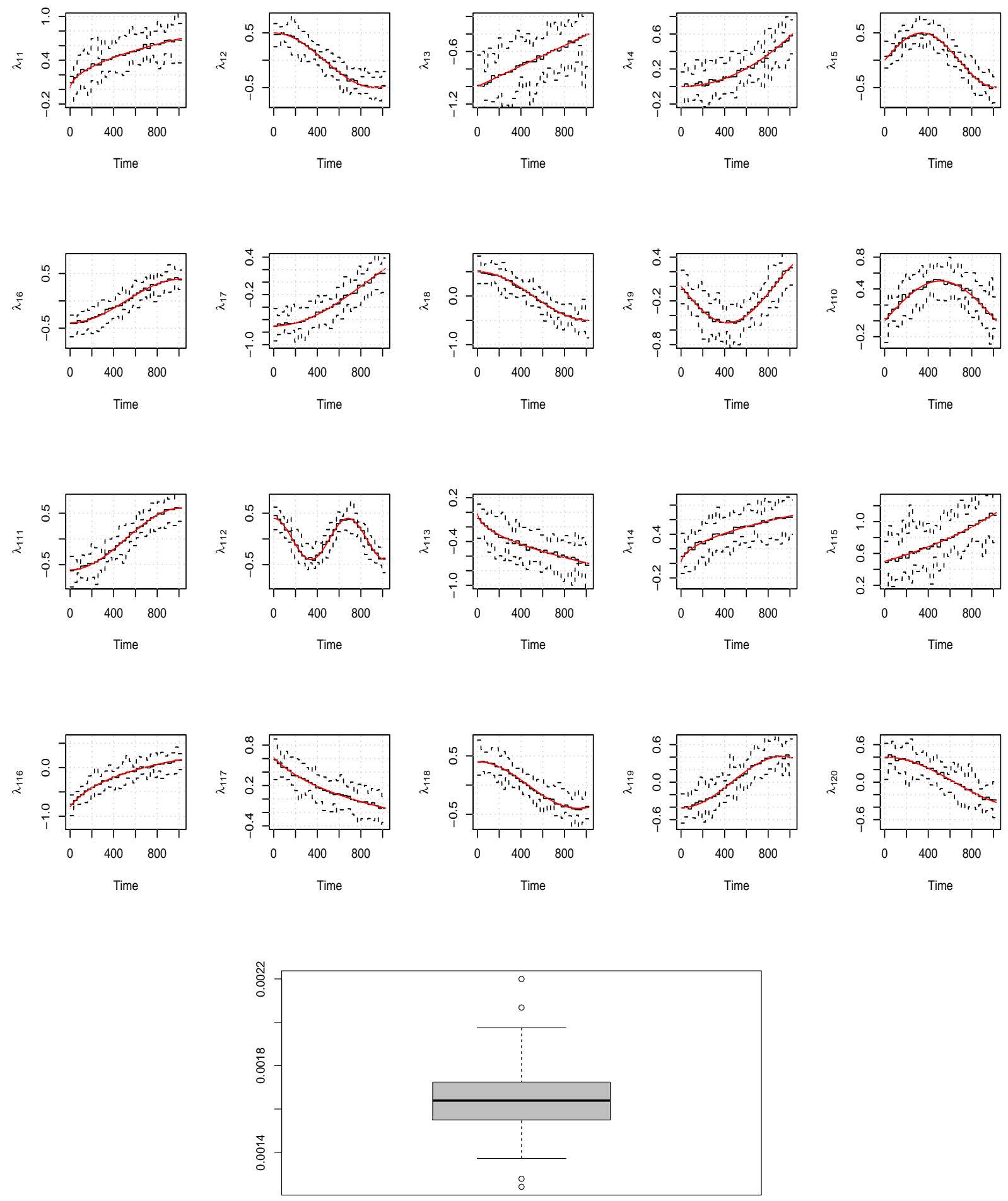

Figura 4.3: Superior: cargas verdadeiras (vermelha), estimadas (escalonada) e linha pontilhada intervalo bootstrap de 1 erro padrão baseado em 100 amostras. Inferior: boxplot dos 1000 EQM das trajetórias das cargas estimadas com ondaleta Haar. Neste caso $\theta=0, \Gamma_{e}=$ Diag, $N=20, T=1024$ e $r=1$. 

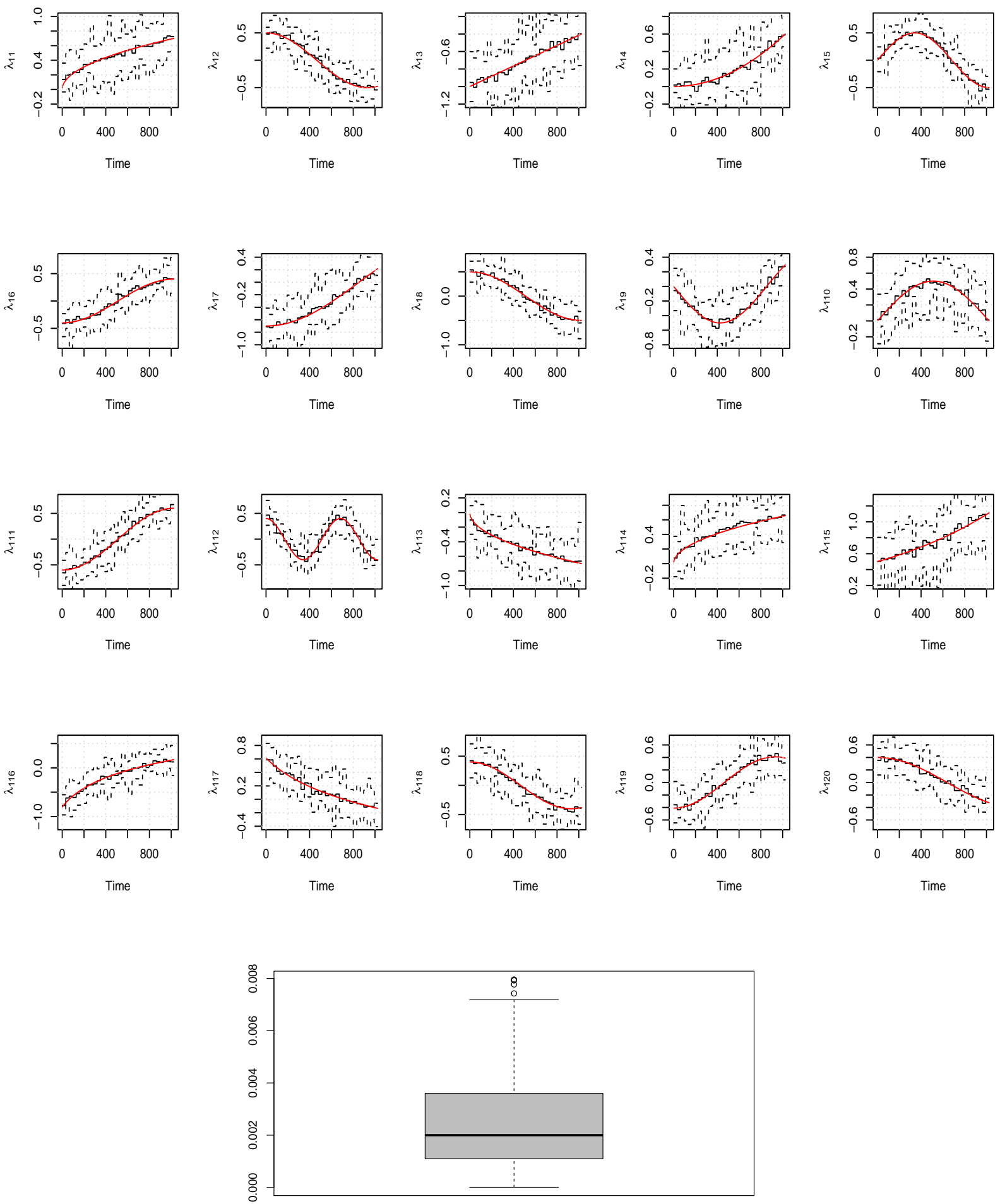

Figura 4.4: Superior: cargas verdadeiras (vermelha), estimadas (escalonada) e linha pontilhada intervalo bootstrap de 1 erro padrão baseado em 100 amostras. Inferior: boxplot dos 1000 EQM das trajetórias das cargas estimadas com ondaleta Haar. Neste caso $\theta=0,5, \Gamma_{e}=$ Diag, $N=20, T=1024$ e $r=1$. 

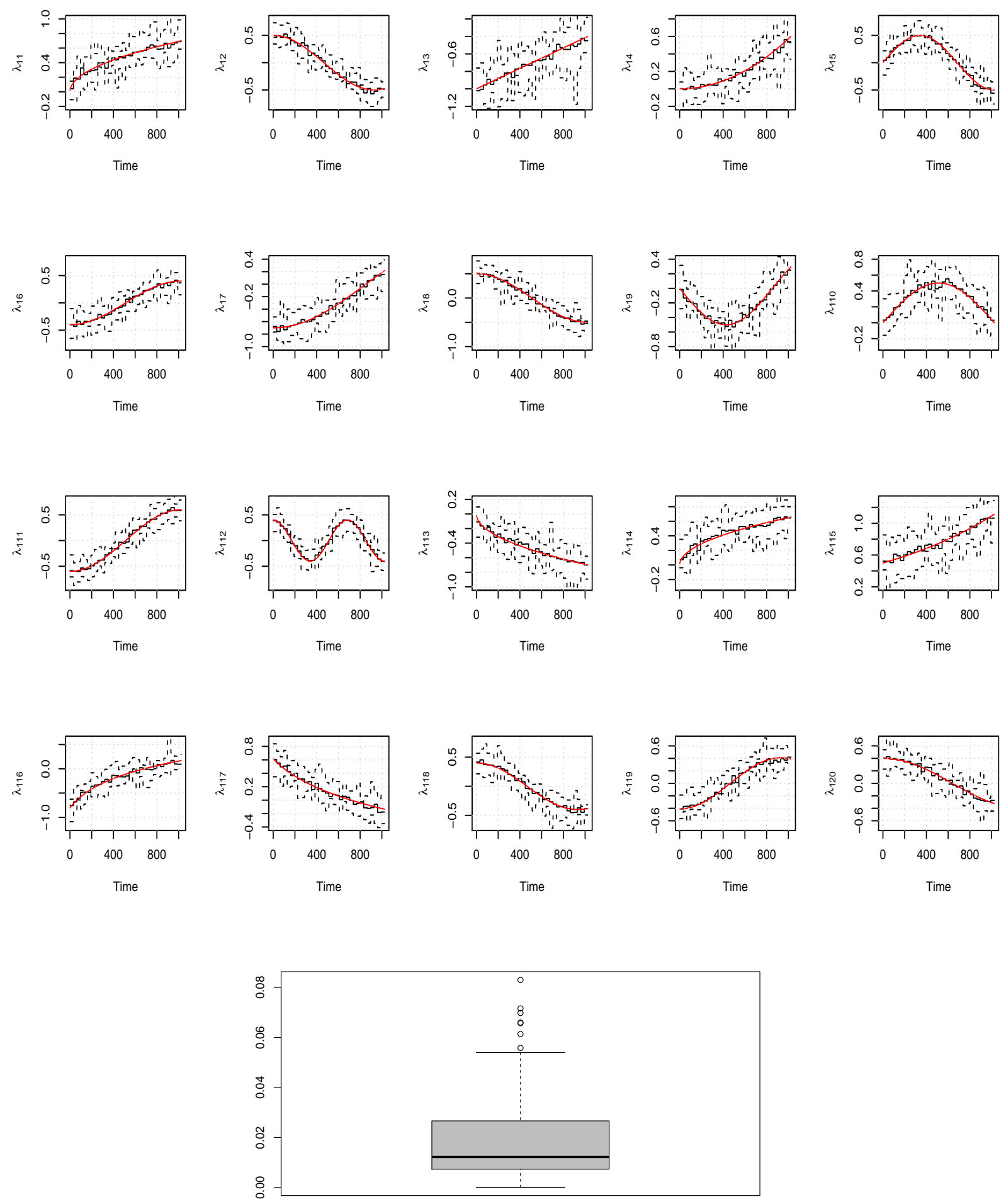

Figura 4.5: Superior: cargas verdadeiras (vermelha), estimadas (escalonada) e linha pontilhada intervalo bootstrap de 1 erro padrão baseado em 100 amostras. Inferior: boxplot dos 1000 EQM das trajetórias das cargas estimadas com ondaleta Haar. Neste caso $\theta=0, \Gamma_{e}=$ Toep, $N=20, T=1024$ e $r=1$. 

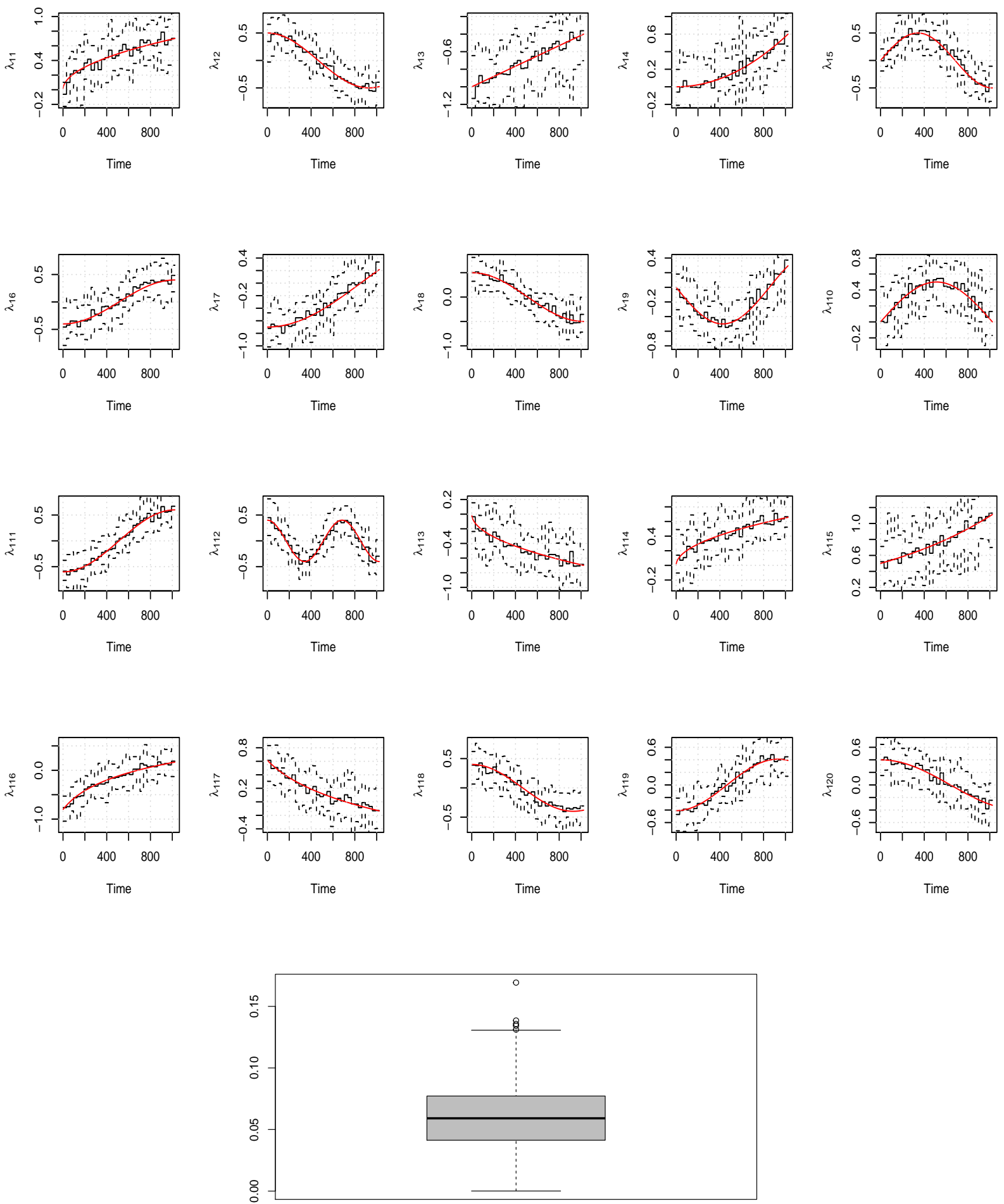

Figura 4.6: Superior: cargas verdadeiras (vermelha), estimadas (escalonada) e linha pontilhada intervalo bootstrap de 1 erro padrão baseado em 100 amostras. Inferior: boxplot dos 1000 EQM das trajetórias das cargas estimadas com ondaleta Haar. Neste caso $\theta=0,5, \Gamma_{e}=$ Toep, $N=20, T=1024$ e $r=1$. 

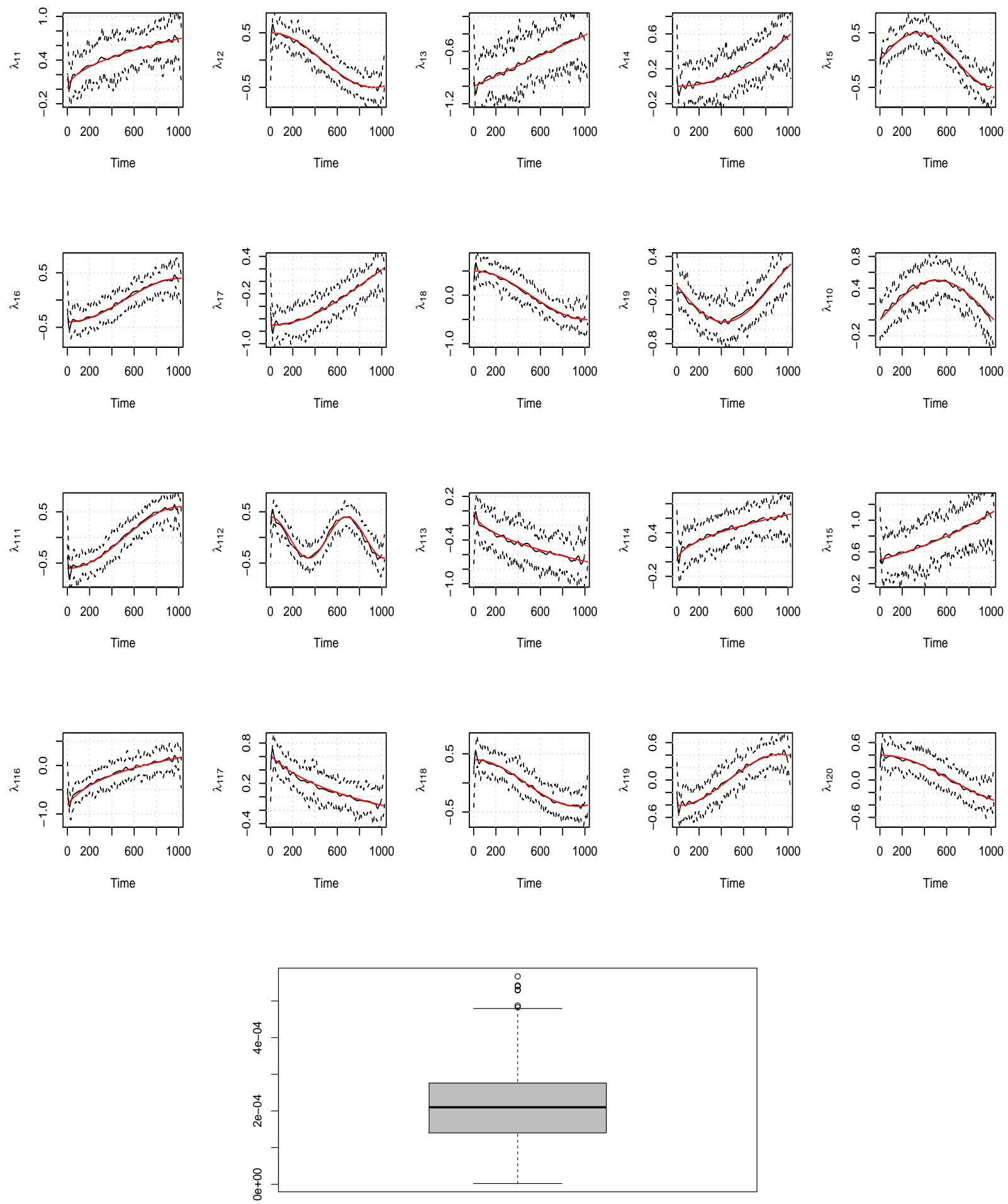

Figura 4.7: Superior: cargas verdadeiras (vermelha), estimadas (escalonada) e linha pontilhada intervalo bootstrap de 1 erro padrão baseado em 100 amostras. Inferior: boxplot dos 1000 EQM das trajetórias das cargas estimadas com ondaleta D8. Neste caso $\theta=0, \Gamma_{e}=$ Diag, $N=20, T=1024$ e $r=1$. 

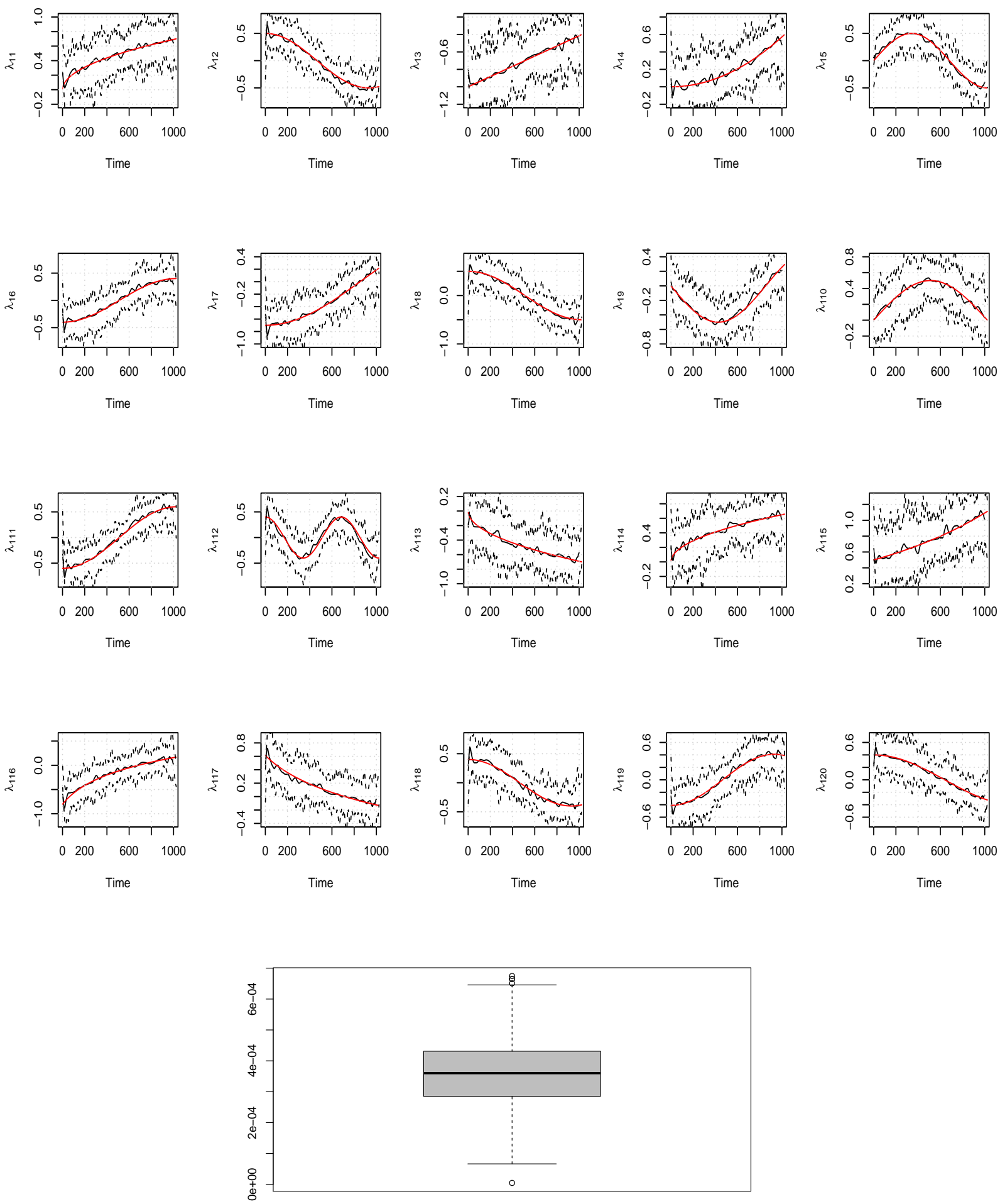

Figura 4.8: Superior: cargas verdadeiras (vermelha), estimadas (escalonada) e linha pontilhada intervalo bootstrap de 1 erro padrão baseado em 100 amostras. Inferior: boxplot dos 1000 EQM das trajetórias das cargas estimadas com ondaleta D8. Neste caso $\theta=0,5, \Gamma_{e}=$ Diag, $N=20, T=1024$ er $=1$. 

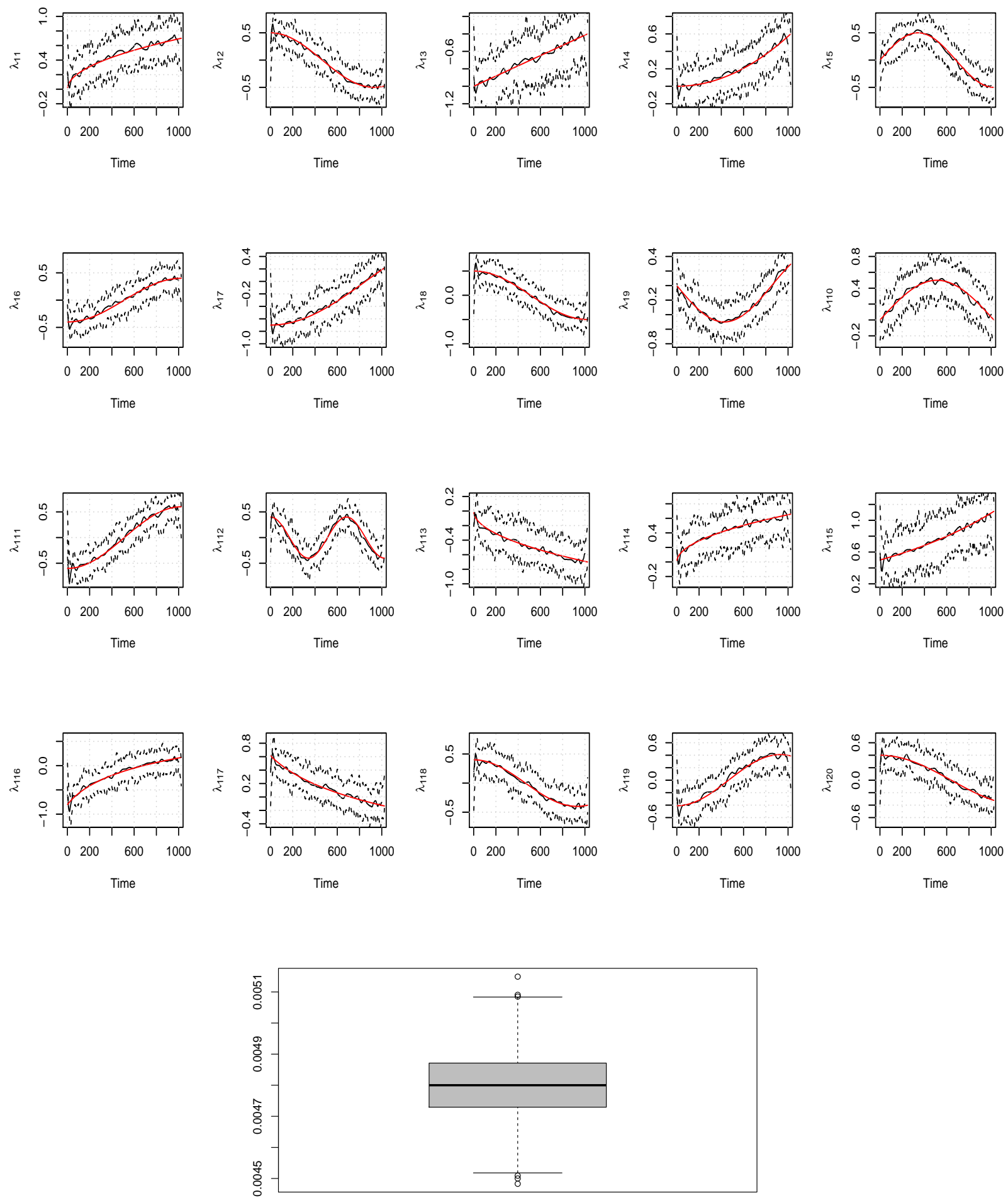

Figura 4.9: Superior: cargas verdadeiras (vermelha), estimadas (escalonada) e linha pontilhada intervalo bootstrap de 1 erro padrão baseado em 100 amostras. Inferior: boxplot dos 1000 EQM das trajetórias das cargas estimadas com ondaleta D8. Neste caso $\theta=0, \Gamma_{e}=$ Toep, $N=20, T=1024$ e $r=1$. 

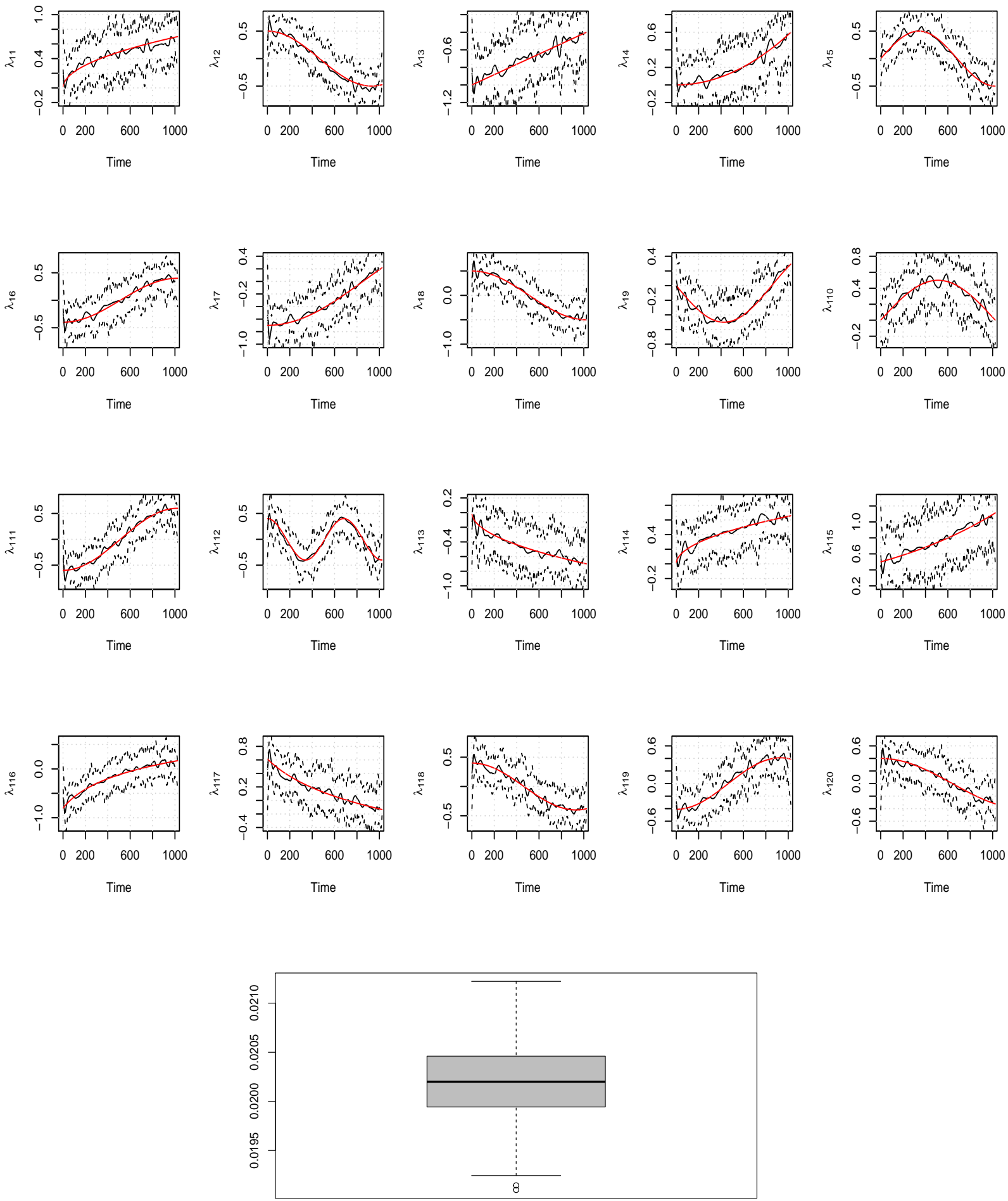

Figura 4.10: Superior: cargas verdadeiras (vermelha), estimadas (escalonada) e linha pontilhada intervalo bootstrap de 1 erro padrão baseado em 100 amostras. Inferior: boxplot dos 1000 EQM das trajetórias das cargas estimadas com ondaleta D8. Neste caso $\theta=0,5, \Gamma_{e}=$ Toep, $N=20, T=1024$ e $r=1$. 

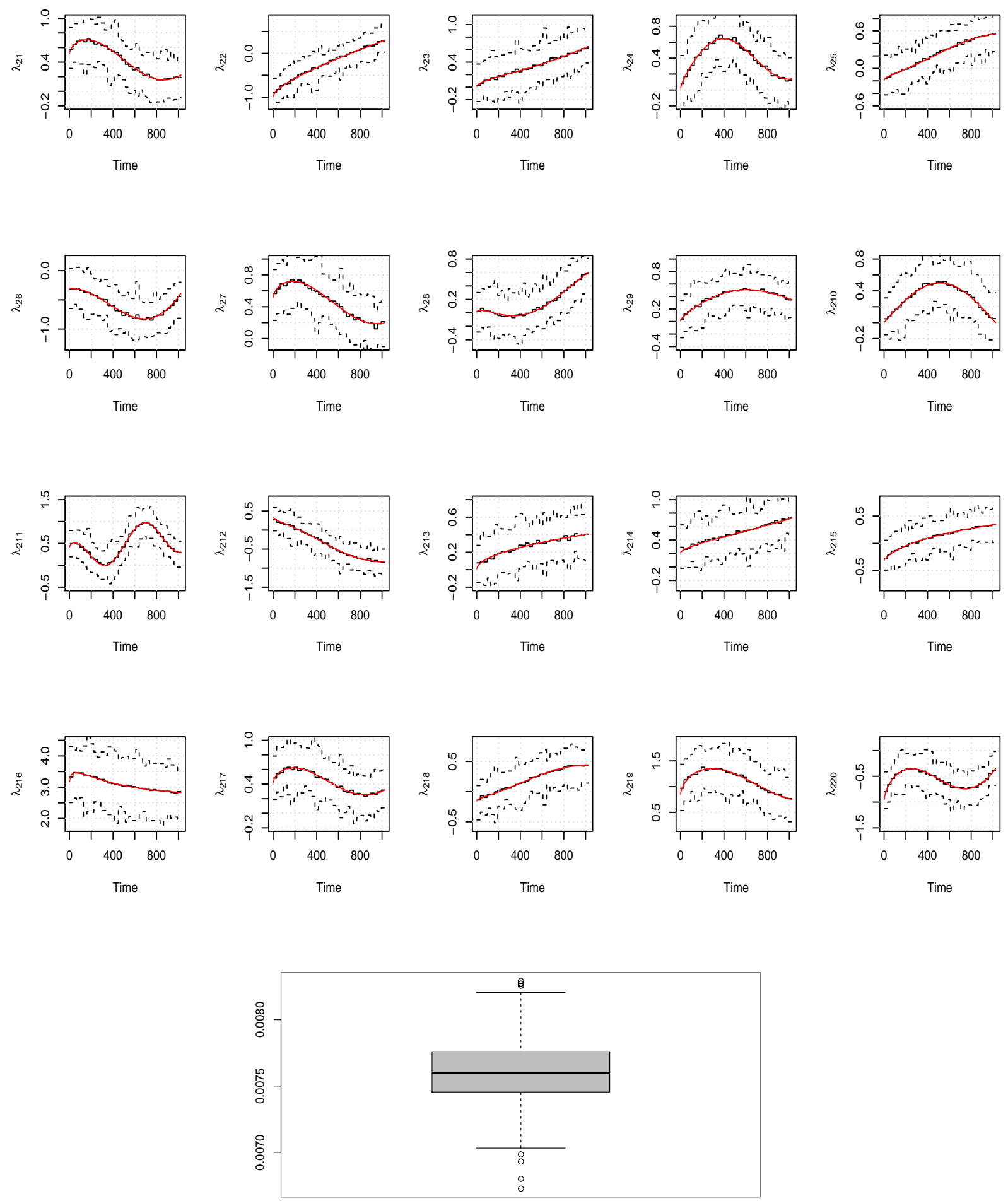

Figura 4.11: Superior: cargas verdadeiras (vermelha), estimadas (escalonada) e linha pontilhada intervalo bootstrap de 1 erro padrão baseado em 100 amostras. Inferior: boxplot dos 1000 EQM das trajetórias das cargas estimadas com ondaleta Haar. Neste caso $\theta=0, \Gamma_{e}=$ Diag, $N=20, T=1024$ e $r=2$. 

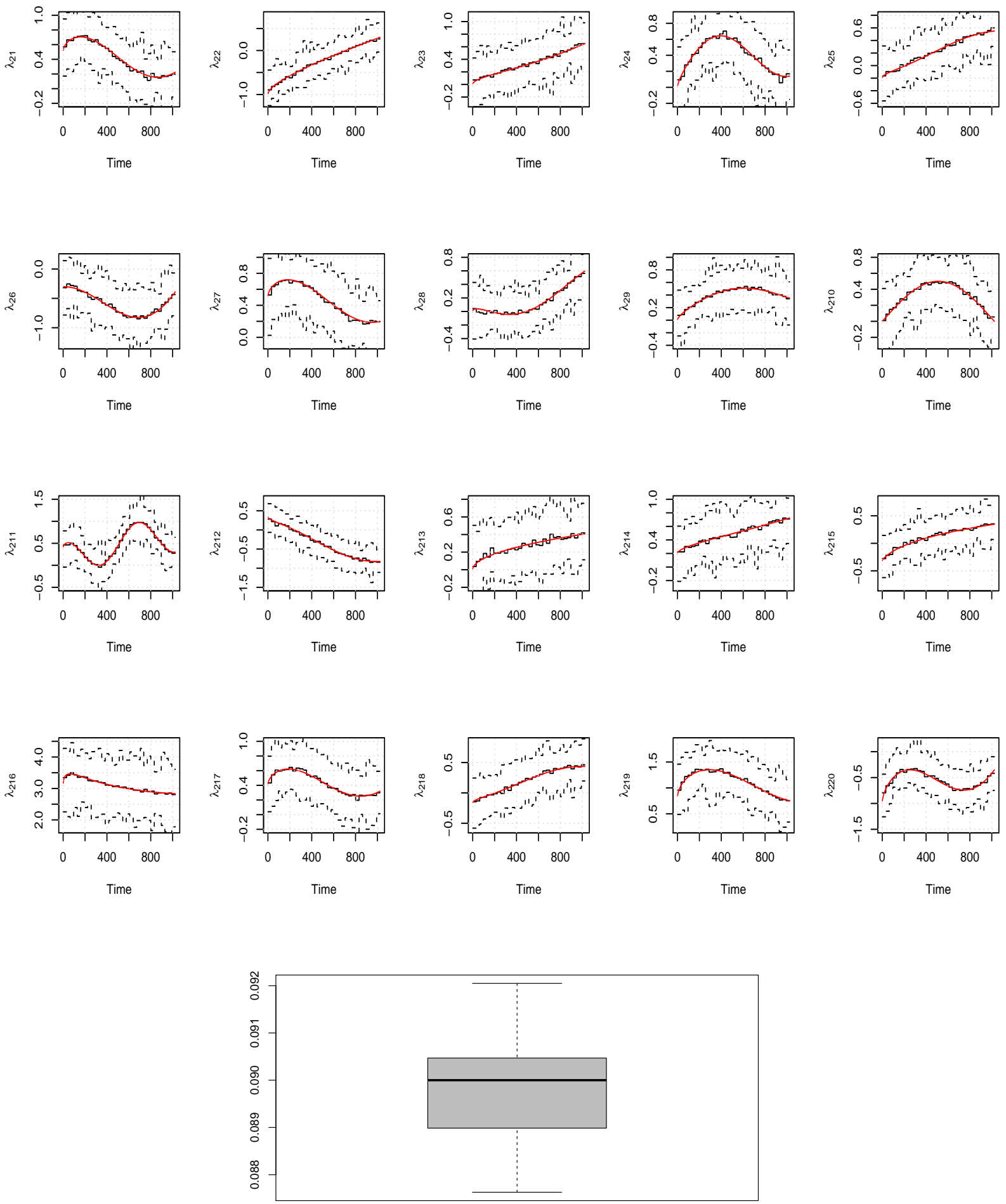

Figura 4.12: Superior: cargas verdadeiras (vermelha), estimadas (escalonada) e linha pontilhada intervalo bootstrap de 1 erro padrão baseado em 100 amostras. Inferior: boxplot dos 1000 EQM das trajetórias das cargas estimadas com ondaleta Haar. Neste caso $\theta=0,5, \Gamma_{e}=$ Diag, $N=20, T=1024$ e $r=2$. 

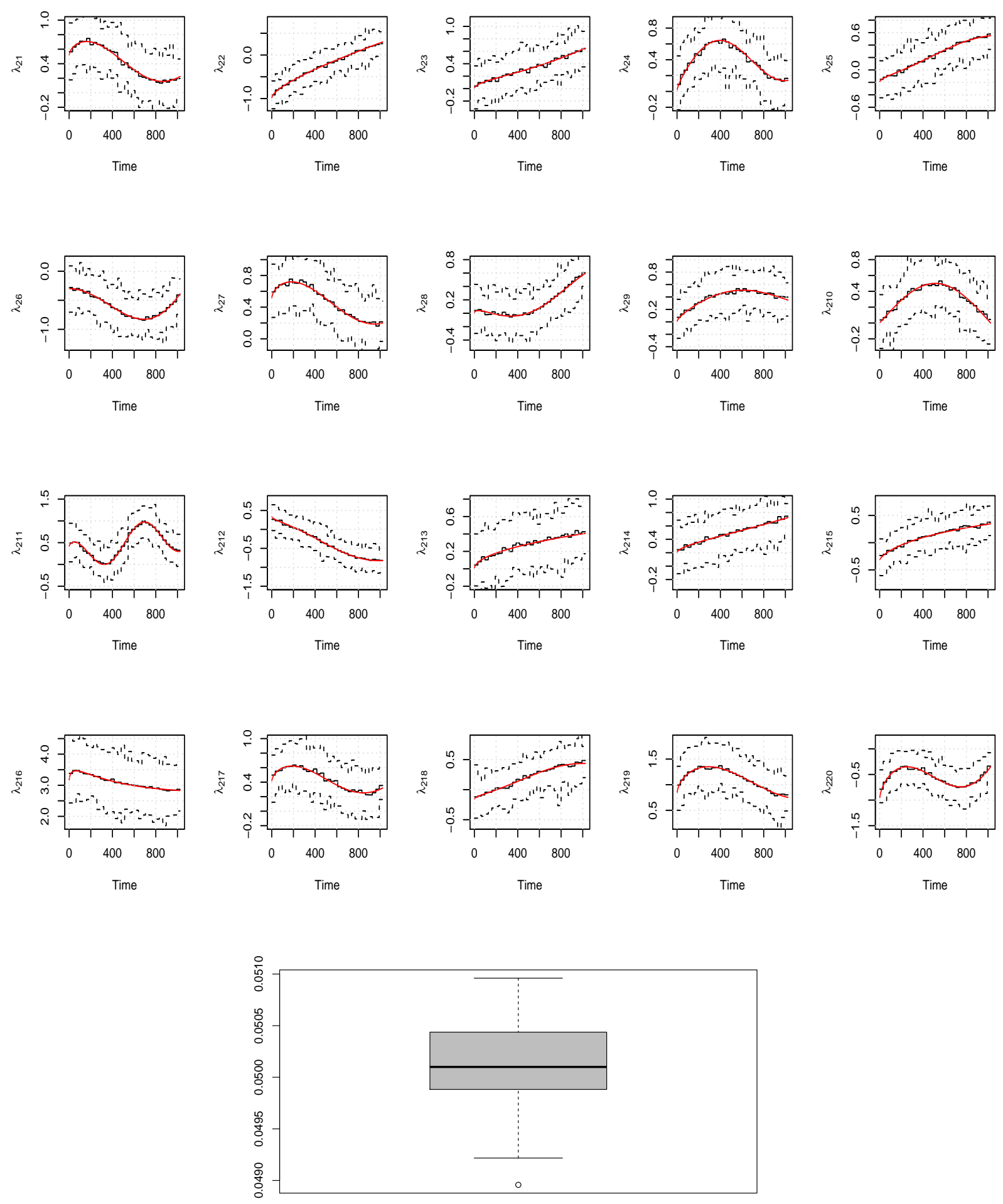

Figura 4.13: Superior: cargas verdadeiras (vermelha), estimadas (escalonada) e linha pontilhada intervalo bootstrap de 1 erro padrão baseado em 100 amostras. Inferior: boxplot dos 1000 EQM das trajetórias das cargas estimadas com ondaleta Haar. Neste caso $\theta=0, \Gamma_{e}=$ Toep, $N=20, T=1024$ e $r=2$. 

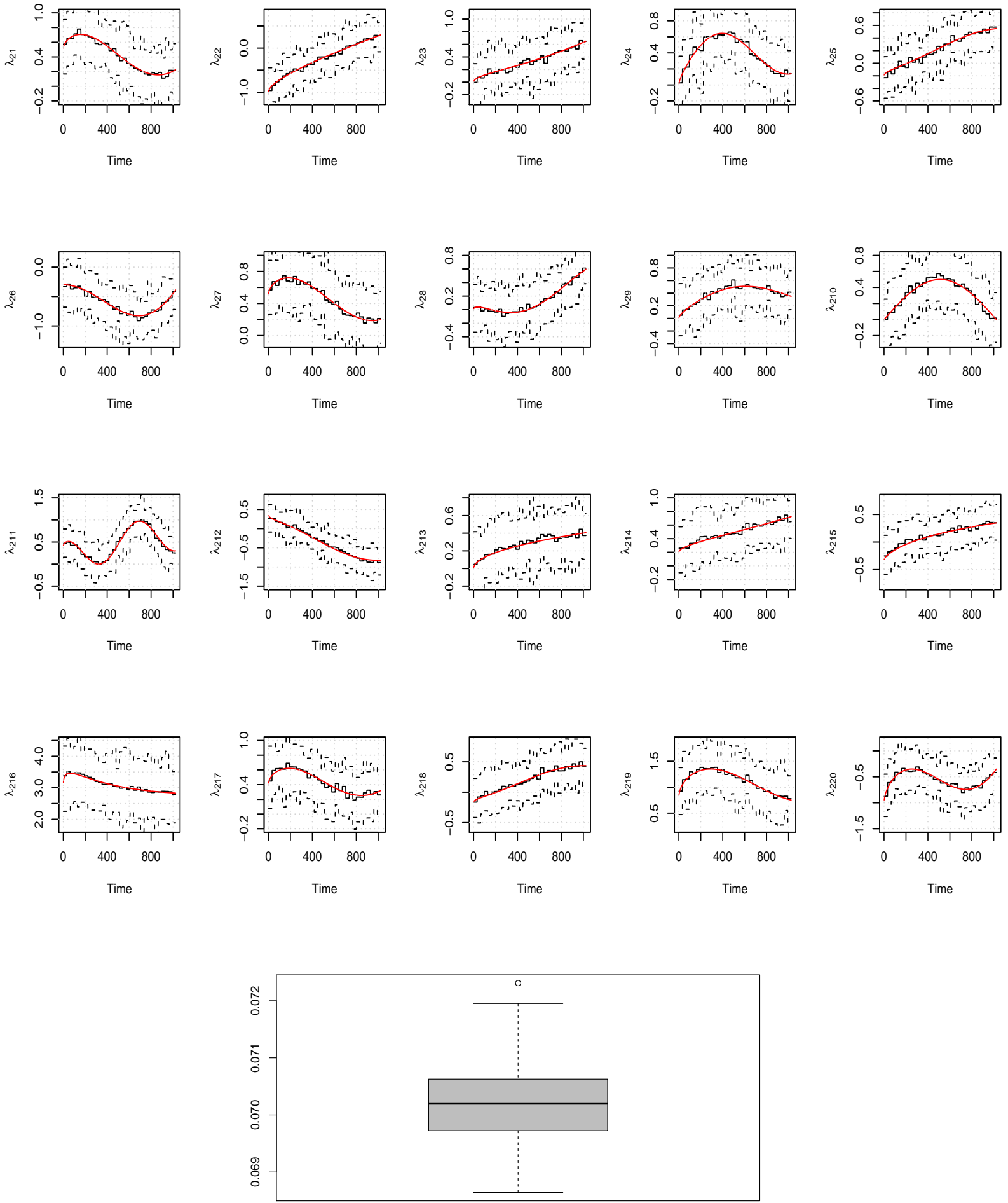

Figura 4.14: Superior: cargas verdadeiras (vermelha), estimadas (escalonada) e linha pontilhada intervalo bootstrap de 1 erro padrão baseado em 100 amostras. Inferior: boxplot dos 1000 EQM das trajetórias das cargas estimadas com ondaleta Haar. Neste caso $\theta=0,5, \Gamma_{e}=$ Toep, $N=20, T=1024$ e $r=2$. 

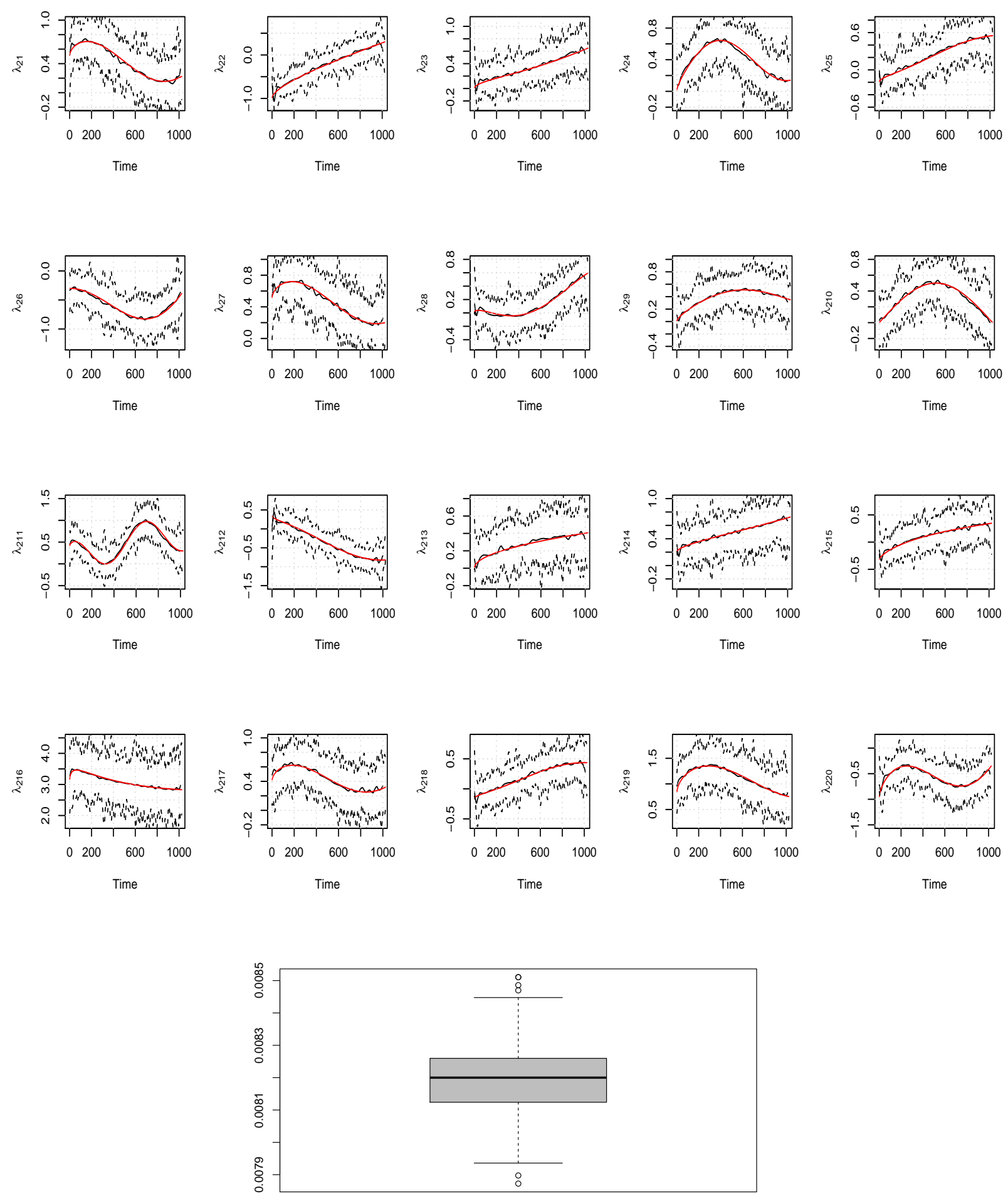

Figura 4.15: Superior: cargas verdadeiras (vermelha), estimadas (escalonada) e linha pontilhada intervalo bootstrap de 1 erro padrão baseado em 100 amostras. Inferior: boxplot dos 1000 EQM das trajetórias das cargas estimadas com ondaleta D8. Neste caso $\theta=0, \Gamma_{e}=$ Diag, $N=20, T=1024$ e $r=2$. 

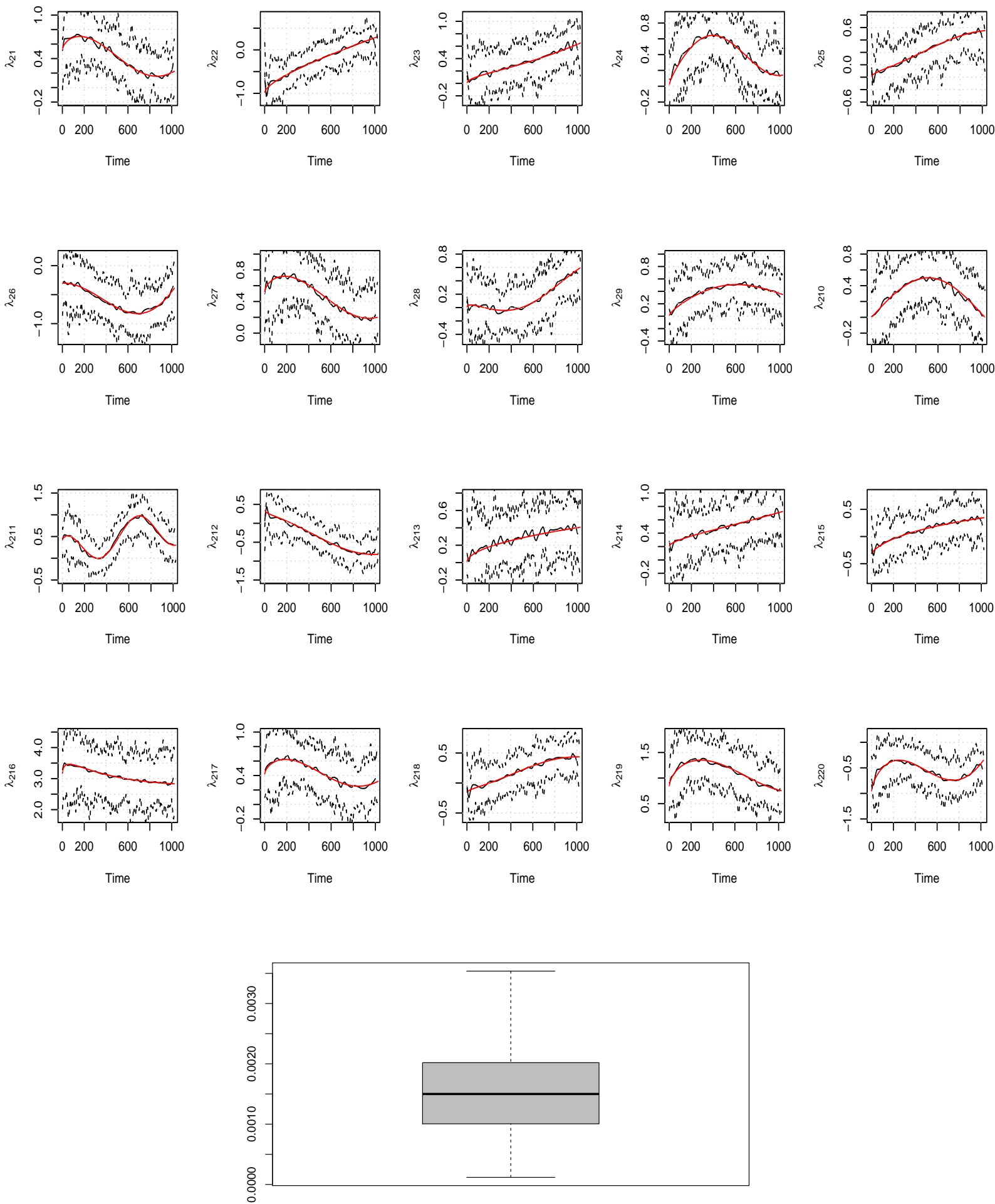

Figura 4.16: Superior: cargas verdadeiras (vermelha), estimadas (escalonada) e linha pontilhada intervalo bootstrap de 1 erro padrão baseado em 100 amostras. Inferior: boxplot dos 1000 EQM das trajetórias das cargas estimadas com ondaleta D8. Neste caso $\theta=0,5, \Gamma_{e}=$ Diag, $N=20, T=1024$ e $r=2$. 

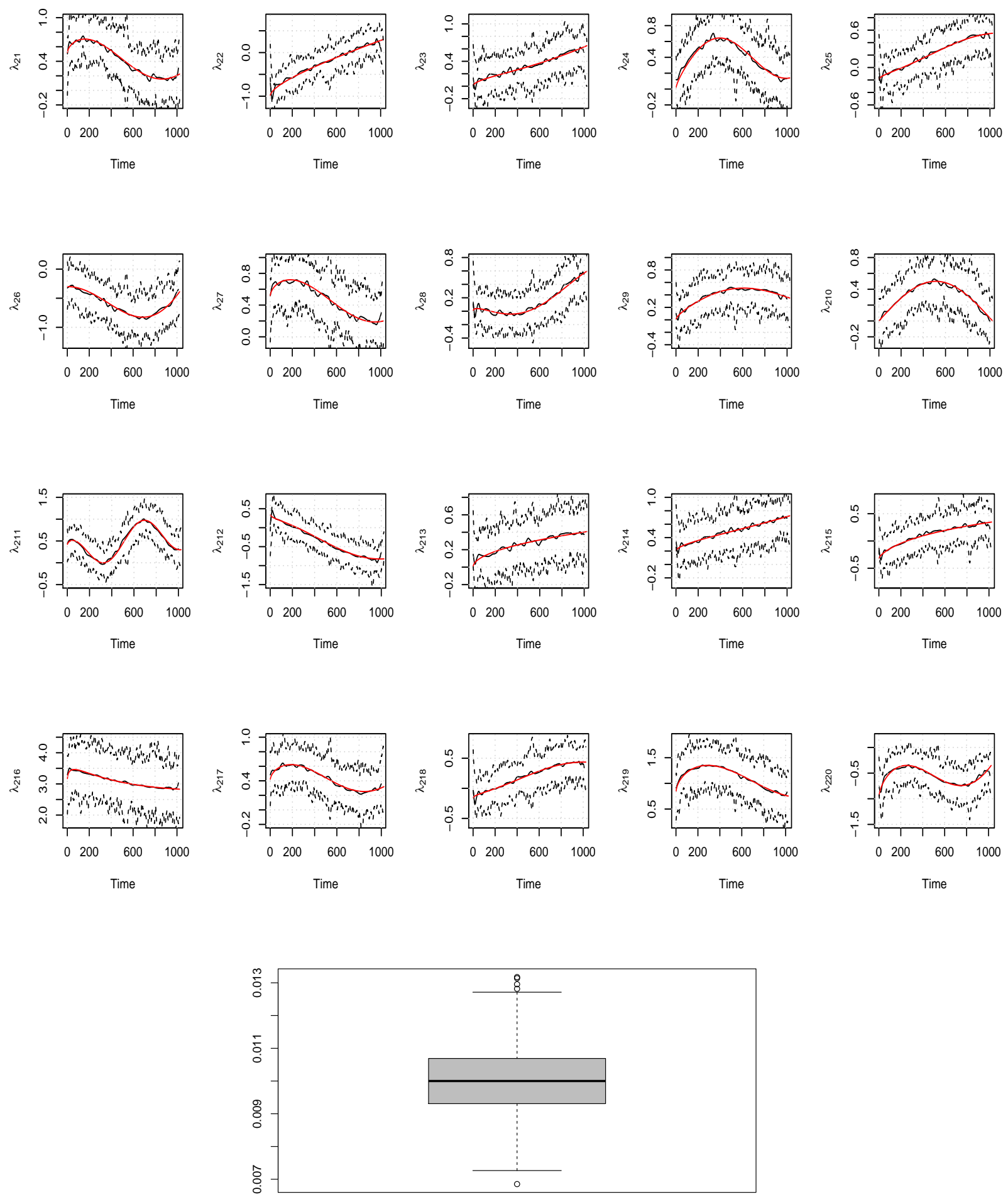

Figura 4.17: Superior: cargas verdadeiras (vermelha), estimadas (escalonada) e linha pontilhada intervalo bootstrap de 1 erro padrão baseado em 100 amostras. Inferior: boxplot dos 1000 EQM das trajetórias das cargas estimadas com ondaleta D8. Neste caso $\theta=0, \Gamma_{e}=$ Toep, $N=20, T=1024$ e $r=2$. 

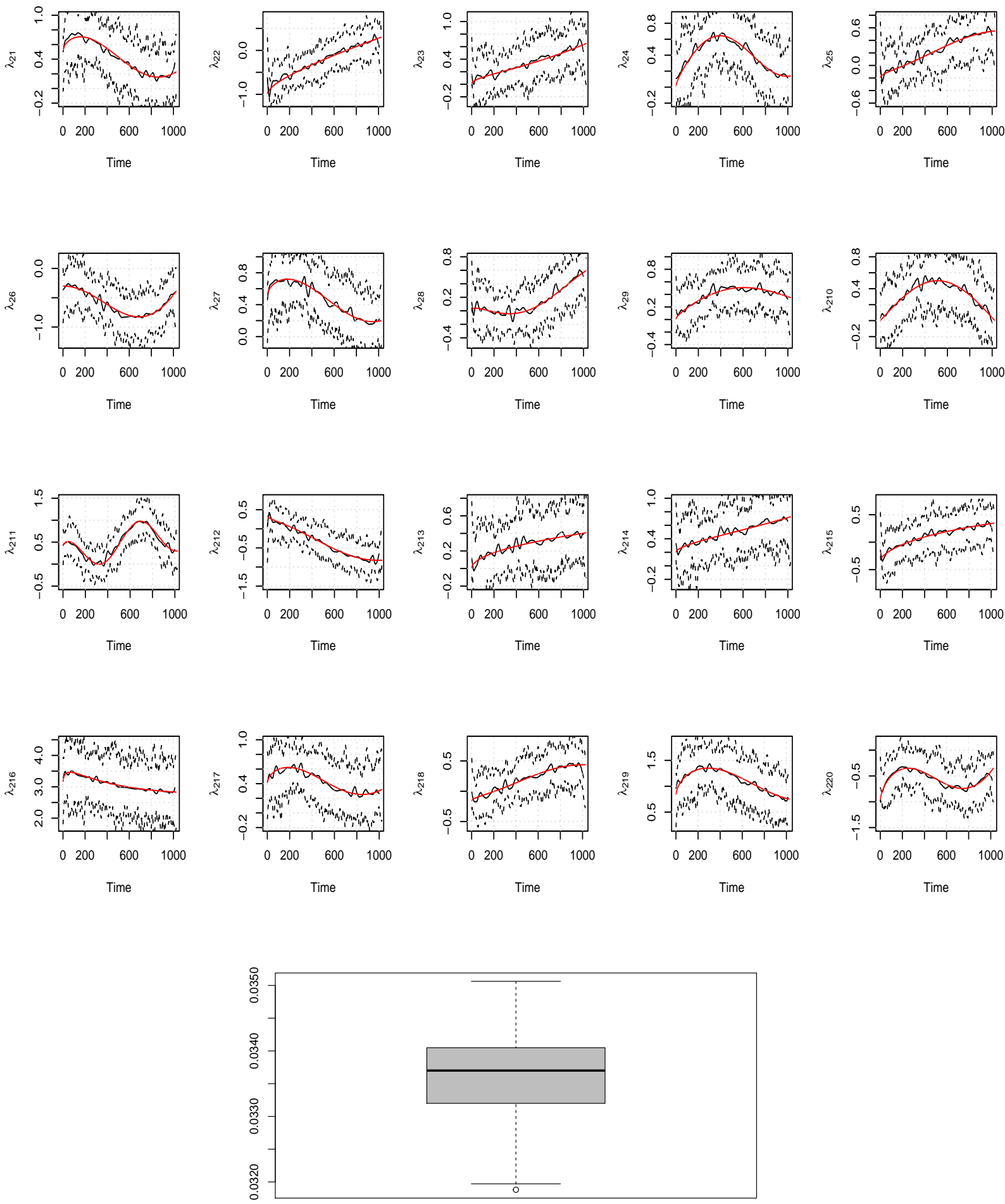

Figura 4.18: Superior: cargas verdadeiras (vermelha), estimadas (escalonada) e linha pontilhada intervalo bootstrap de 1 erro padrão baseado em 100 amostras. Inferior: boxplot dos 1000 EQM das trajetórias das cargas estimadas com ondaleta D8. Neste caso $\theta=0,5, \Gamma_{e}=$ Toep, $N=20, T=1024$ e $r=2$. 


\subsection{Comentários}

- O estimador dos CP apresenta bom desempenho quando as funções são tais que $|\lambda(t)|<1$. Nos casos que essas funções apresentam mudanças abruptas e maior variabilidade, esses fatores estimados afetam diretamente na estimação dos coeficientes de ondaletas e como consequência às cargas estimadas.

- Construindo os intervalos de confiança bootstrap para o caso de erros idiossincráticos independentes e fatores sem correlação serial, observamos que eles apresentam pouca variabilidade em relação ao caso em que existe dependência nos erros idiossincráticos e fatores serialmente correlacionados.

- O EQM em todos os casos usando ondaleta D8 é menor em relação ao EQM usando ondaleta Haar. No entanto, ao fazer o processo de estimação com D8, tivemos problemas nas bordas das cargas, o qual foi solucionado empregando ondaletas periódicas através da função $d w t(\cdot)$ do R.

- O fato de estimar os fatores supondo que as cargas são constante ao longo do tempo não é problema, pois a não estacionaridade em covariância é capturada pelas cargas variando no tempo. Os fatores podem ser vistos como eixos principais de similaridade com os dados observados, enquanto que as cargas capturam essas mudanças em covariância.

- Em geral, o método tem bom desempenho para os cenários considerados neste trabalho, conseguindo estimar a variação geral das cargas fatoriais. Adicionalmente podemos observar que para essas funções o desempenho da ondaleta D8 foi melhor. Portanto consideramos a metodologia proposta como uma alternativa para o estudo da análise fatorial em séries temporais multivariadas que são não estacionárias de segunda ordem e conduzidas por co-movimentos suaves em sua estrutura. 


\section{Capítulo 5}

\section{Aplicação}

Nesta seção, fornecemos uma aplicação da metodologia proposta para estudar os preços no mercado spot de energia do Nord Pool.

\subsection{Descrição dos dados}

Ao longo das últimas décadas, surgiu uma liberalização dos mercados de energia. As empresas de energia produzem energia elétrica de várias fontes diferentes (sistemas hidrelétricos, térmicos, nucleares, eólicos e solares), a fim de fornecer preços competitivos e garantir a eficiência da produção. De ponto de vista econômico, os mercados da eletricidade procuram combinar a oferta e a demanda para encontrar um preço de compensação do mercado.

Tais preços apresentam fatores cíclicos irregulares que estão associados a movimentos cíclicos na economia ou tendências climáticas de longo prazo, enquanto que vários pontos são causados por algumas datas especiais antecipadas (Natal, feriados nacionais, etc.) e dias inesperados originados intrinsecamente no mercado. Weron (2007) relata esses fatos estilizados, bem como uma visão geral dos métodos estatísticos utilizados na literatura.

Portanto é necessário examinar em detalhe os preços em cada hora da eletricidade, e implica o estudo de uma complexa estrutura de dependência no mercado. Uma maneira natural de levar em consideração essa dependência é com uma abordagem tipo VAR, no entanto, nos conduz ao problema da "maldição da dimensionalidade"e, portanto, é de interesse reduzir a dimensão. Neste contexto os modelos fatoriais são ferramentas mais adequadas para analisar dados dimensionais elevados, os quais foram usados recentemente em mercados de eletricidade (ver, por exemplo, Alonso et al. (2011), Dordonnat et al. (2012) e Raviv et al. (2015)).

No presente trabalho, o conjunto de dados em consideração consiste em $N=24$ preços a cada hora diariamente durante o período de 6/08/2016 até 31/12/2017 e, portanto, produz um total de $T=513$ observações para cada série observada em determinada hora do dia. Os preços são denominados em euros por Mwh de carga $y_{i t}$ para $i=1, \ldots, 24$, em que $y_{i t}$ representa o preço no $i$ - ésima hora do $t-$ 
ésimo dia. Seguindo Ergemen et al. (2015), a série é pré-filtrada por

$$
y_{i t}=\alpha_{i 0}+\alpha_{i 1} t+\alpha_{i 2} D_{t}+\mathbb{B}_{t}^{\prime} A_{i}+\alpha_{i 3} \cos \frac{2 \pi t}{365}+\alpha_{i 4} \cos \frac{2 \pi t}{7}+\alpha_{i 5} \cos \frac{2 \pi t}{3.5}+y_{i t}^{*}
$$

em que $\mathbb{B}_{t}$ é um vetor de variáveis dummies, as quais capturam níveis de mudanças causadas por quebras estruturais. $D_{t}$ é uma variável dummie para os feriados que tem valor 1 se alguns dos países participantes no sistema Nord Pool suspendem ou reduzem as atividades comerciais normais por costume ou lei, e 0 de outros casos. Neste trabalho aplicamos a metodologia proposta para $\log y_{i t}^{*}$, cujo gráfico por hora ao longo dos 513 dias é apresentado na Figura 5.1.

H1

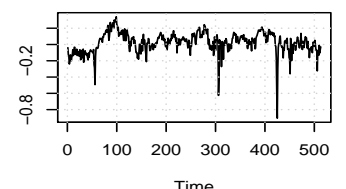

H5

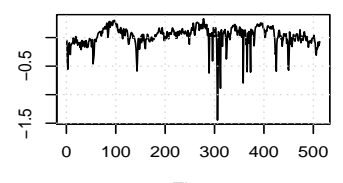

Time

H9

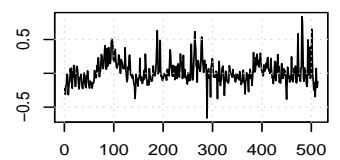

Time

H13

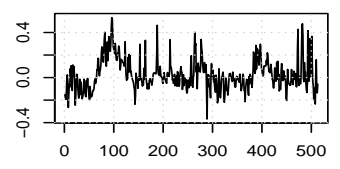

Time

H17

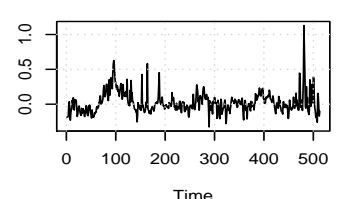

H21

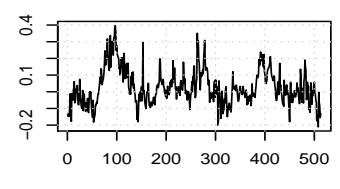

H2

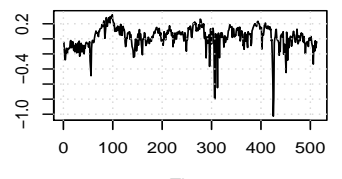

H6

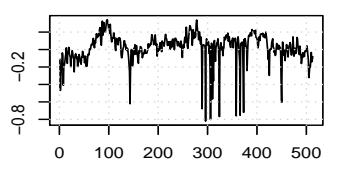

Time

H10

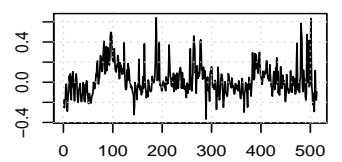

Time

H14

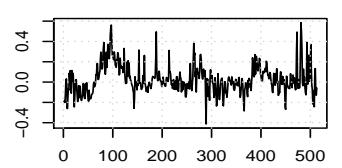

Time

H18

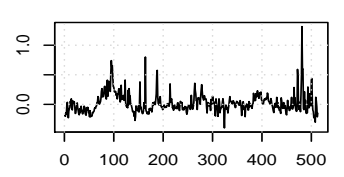

Time

H22

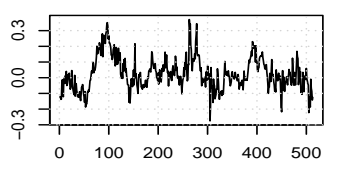

H3

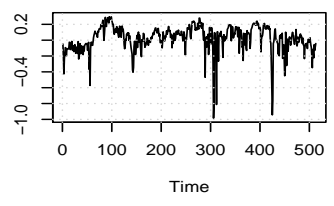

H7

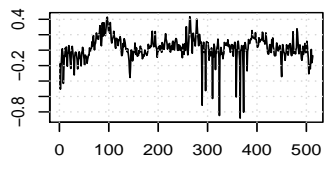

Time

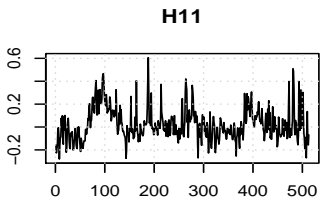

Time

H15

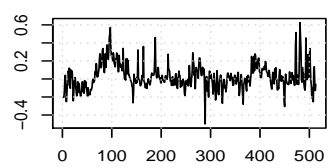

Time

H19

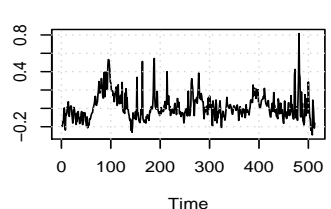

H23

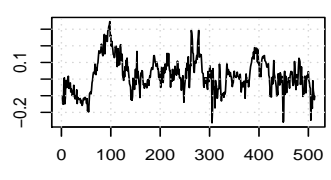

H4

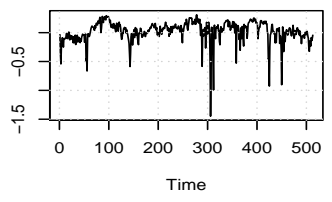

H8

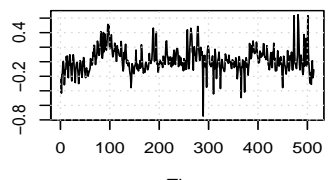

Time

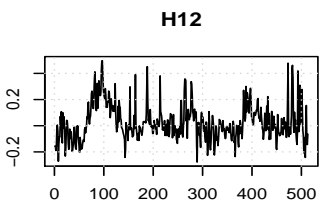

Time

H16

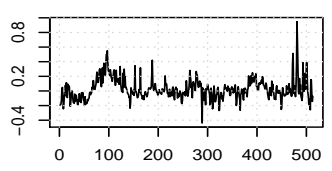

Time

H20
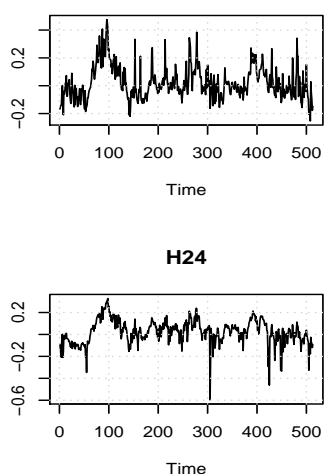

Figura 5.1: Logaritmo dos preços de energia por hora ao longo dos 513 dias 


\subsection{Teste para não estacionariedade}

Antes de aplicar o modelo ao conjunto de dados, consideramos um teste para não estacionariedade para modelos fatoriais estáticos. O teste é proposto por Hoffman e Pagan (1989) que compara a variância de duas diferentes subamostras das séries observadas, sua base é o estimador da variância amostral e autocovariâncias dos segundos momentos das séries observadas.

O teste começa dividindo a amostra em duas partes

$$
\begin{aligned}
\left\{Y_{i t}^{(1)}\right\} & =Y_{i 1}, \ldots, Y_{i T_{1}} \\
\left\{Y_{i t}^{(2)}\right\} & =Y_{i\left(T_{1}+1\right)}, \ldots, Y_{i T}
\end{aligned}
$$

para $i=1, \ldots, N$, assim $T=T_{1}+T_{2}$ com $T_{1}=k_{T} T_{2}$. A hipóteses nula de variância incondicional constante nas duas partes é dada por

$$
H_{0}: \operatorname{Var}\left(Y_{i t}^{(1)}\right)=\operatorname{Var}\left(Y_{i t}^{(2)}\right)
$$

e pode ser testado com a diferença entre as duas variâncias amostrais $\Delta_{i}=\hat{\sigma}_{i T_{1}}-\hat{\sigma}_{i T_{2}}$.

Se existe um $k \in(0,1)$ tal que $k_{T} \rightarrow k$ quando $T \rightarrow \infty$, a variância ao longo prazo do quadrado das observações $\left\{Y_{i t}^{2}, t=1, \ldots, T\right\}$ é dada por

$$
\nu_{i}^{2}=\gamma_{i 0}+2 \sum_{j=1}^{\infty} \gamma_{i j}
$$

em que $\gamma_{i j}=\operatorname{Cov}\left(Y_{i, t}^{2}, Y_{i, t+j}^{2}\right)$ para $i=1, \ldots, N$. O teste é baseado na convergência sob $H_{0}$,

$$
\sqrt{T_{1}} \Delta_{i} \stackrel{d}{\longrightarrow} \mathcal{N}\left(0,(1+k) \nu_{i}^{2}\right)
$$

e a estatística do teste é dada por

$$
H P=\sqrt{\frac{T_{1}}{\left(1+k_{T}\right) \hat{\nu}_{i}^{2}}} \Delta_{i}
$$

$H P$ tem distribuição assintoticamente normal com média zero e variância um, $\mathcal{N}(0,1)$. O estimador de $\nu_{i}^{2}$ é obtido através de

$$
\hat{\nu}_{i}^{2}=\hat{\gamma}_{i 0}+\sum_{j=1}^{Q}\left(1-\frac{j}{Q}\right) \hat{\gamma}_{i j},
$$

em que $\hat{\gamma}_{i j}$ é a autocovariância amostral de $Y_{i t}^{2}$, e o parâmetro $Q$ é da ordem $T^{1 / 4}$. 
H1

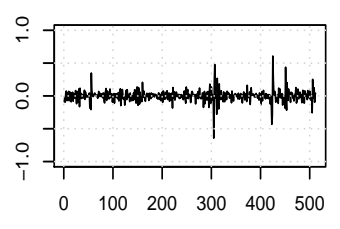

Time

H5

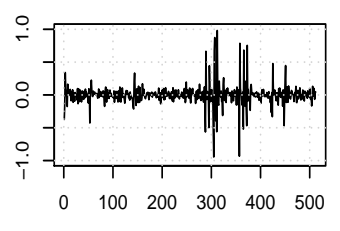

Time

H9

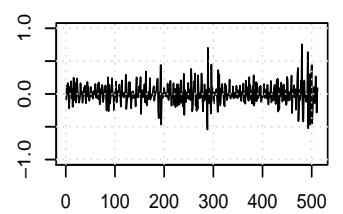

Time

H13

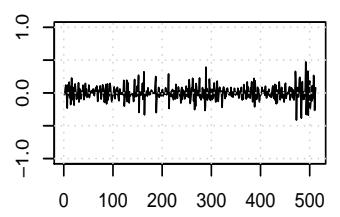

Time

H17

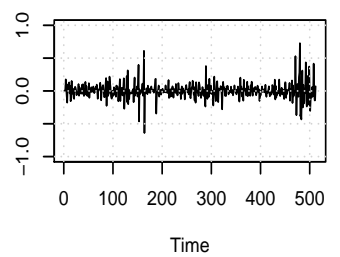

H21

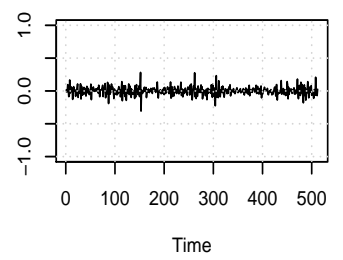

H2

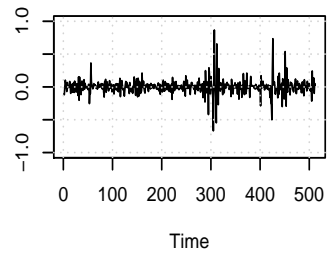

H6

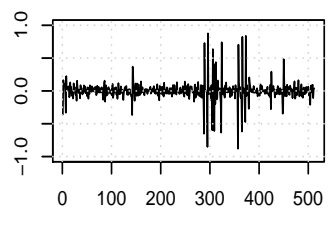

Time

H10

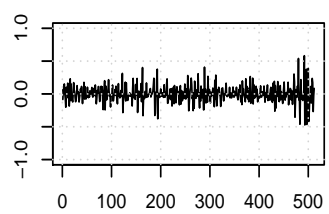

Time

H14

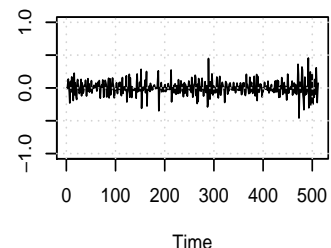

H18

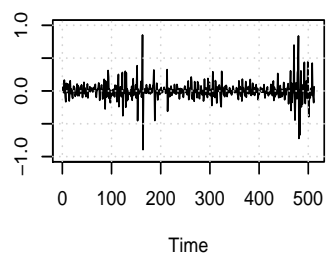

H22

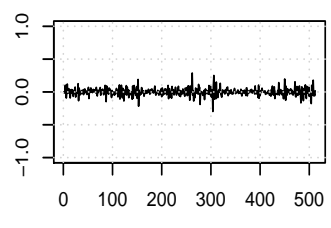

H3

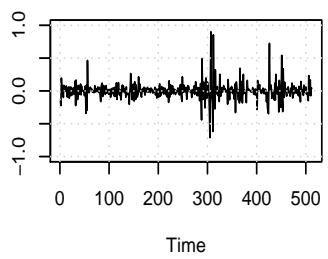

H7

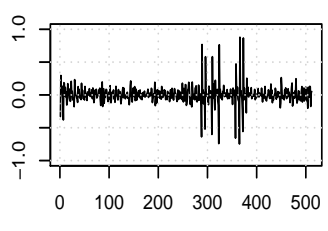

Time

H11

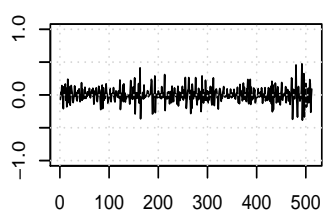

Time

H15

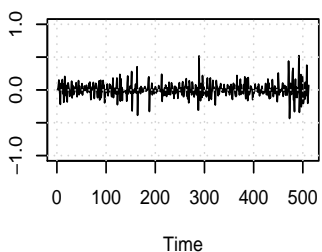

H19

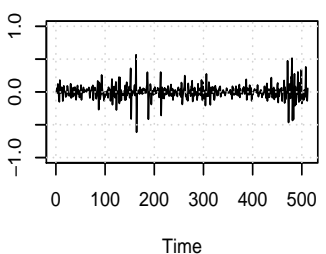

H23

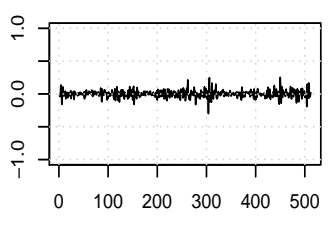

H4

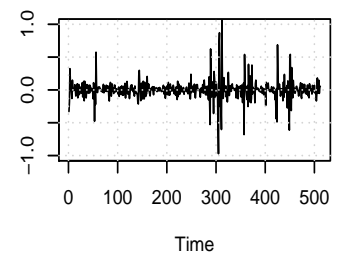

H8

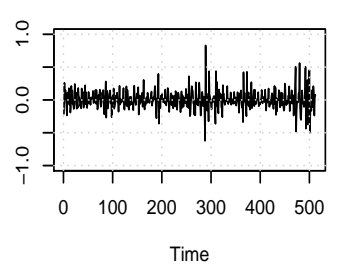

H12

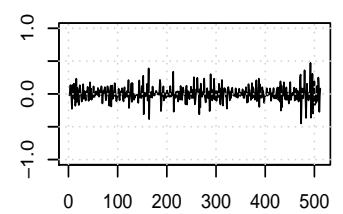

Time

H16

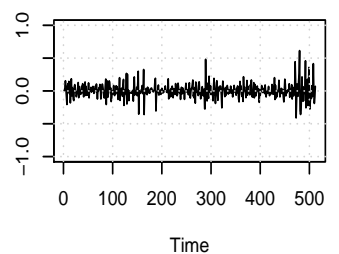

H20

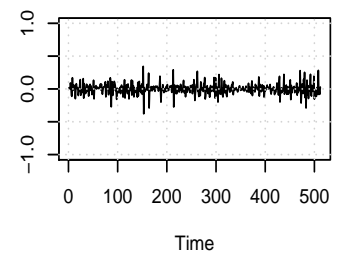

H24

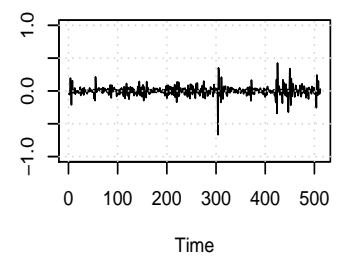

Figura 5.2: Log-retorno dos preços de energia por hora ao longo dos 513 dias 
As séries de log-retorno dos preços de energia são apresentados na Figura 5.2, podemos observar a existência de estrutura de segunda ordem variando no tempo e co-movimentos na maioria das séries. Para avaliar estatisticamente essa observação, aplicamos o teste para cada série separadamente com um nível de $\alpha=0,05$ e $k_{T}=1$. Entre as 24 séries analisadas $N=24$, o teste rejeitou $H_{0}$ para 13 séries. Também foi aplicado um teste global via correção de Bonferroni com um nível $\beta=\alpha / N=0,05 / 24=$ 0.00208 , no qual rejeitamos $H_{0}$. Os valores da estatística são apresentados na Figura 5.3. De acordo com os resultados do teste, temos evidência de não estacionaridade de segunda ordem, motivo pelo qual procedemos na aplicação do modelo proposto nesse conjunto de dados.

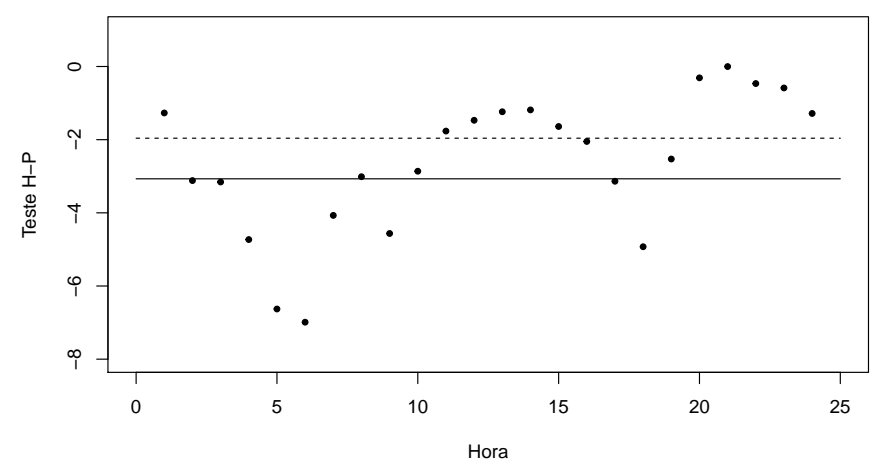

Figura 5.3: Valores da estatística de teste HP definida em (5.2) (pontos pretos), valores críticos $\pm z_{\alpha / 2}= \pm 1,96$ (linhas tracejadas) $e \pm z_{\beta / 2}= \pm 3,07$ (linha contínua). O teste foi aplicado a cada série separadamente $(\alpha=0,05)$, bem como a todo o conjunto de séries através da correção de Bonferroni $(\beta=\alpha / N)$.

\subsection{Estimação}

Consideremos (3.8) para modelar o conjunto de séries. Em um primeiro estágio, estimamos $\mathbf{F}_{t}$ usando os CP. Pelo scree plot apresentado na Figura 5.4, 80\% da variabilidade das 24 séries são representadas pelos 2 primeiros fatores, portanto esses dois serão considerados para o segundo estágio.

\section{Scree plot}

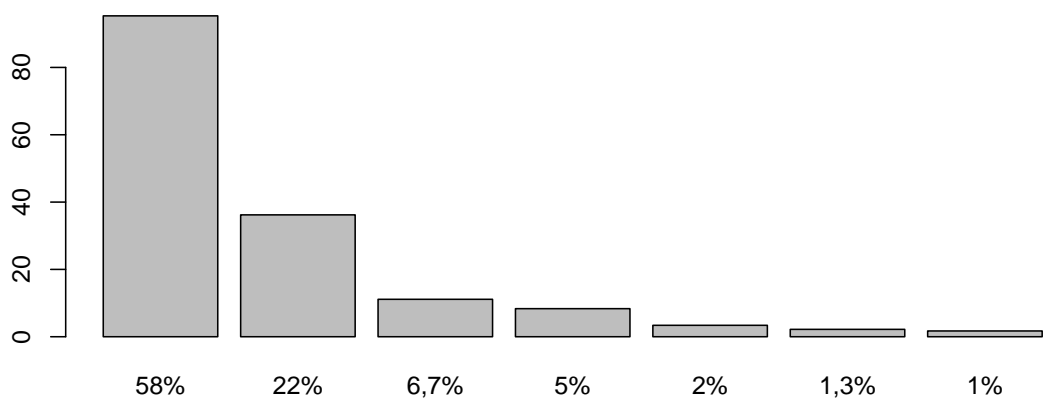

Figura 5.4: Porcentagem explicado por cada $C P$ 
Podemos notar na Figura 5.5 que o primeiro fator estimado $\hat{\mathbf{F}}_{1 t}$ representa aquelas séries que apresentam uma variabilidade significativa mas estável e dois pequenos agrupamentos em torno dos instantes $t=300$ e $t=500$, especificamente as séries H9-H16 e H20-H23. Enquanto que o segundo fator reflete aquelas séries que possuem um agrupamento notável em torno do instante $t=300$, com uma volatilidade fraca no período $t<300$.
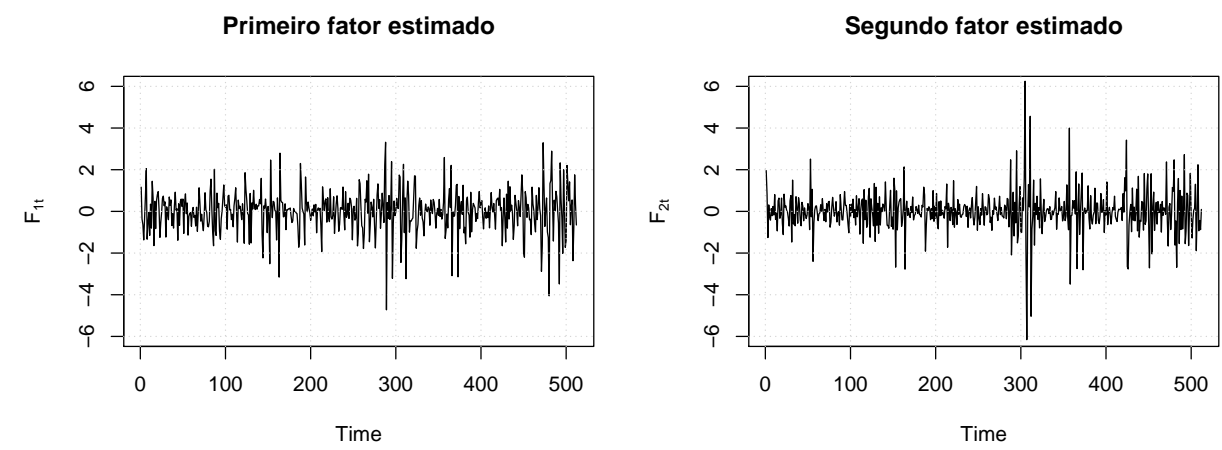

Figura 5.5: Fatores estimados

No segundo estágio, as cargas funcionais são estimadas usando (3.18), as ondaletas Haar e D8 com um nível de resolução $J=5$ foram empregadas. Nas Figuras (5.6) e (5.7) apresentamos as cargas fatoriais estimadas empregando dois fatores, ondaleta Haar e incluindo intervalos bootstrap de um erro padrão. Enquanto que nas Figuras (5.8) e (5.9) foi utilizada a ondaleta D8. 


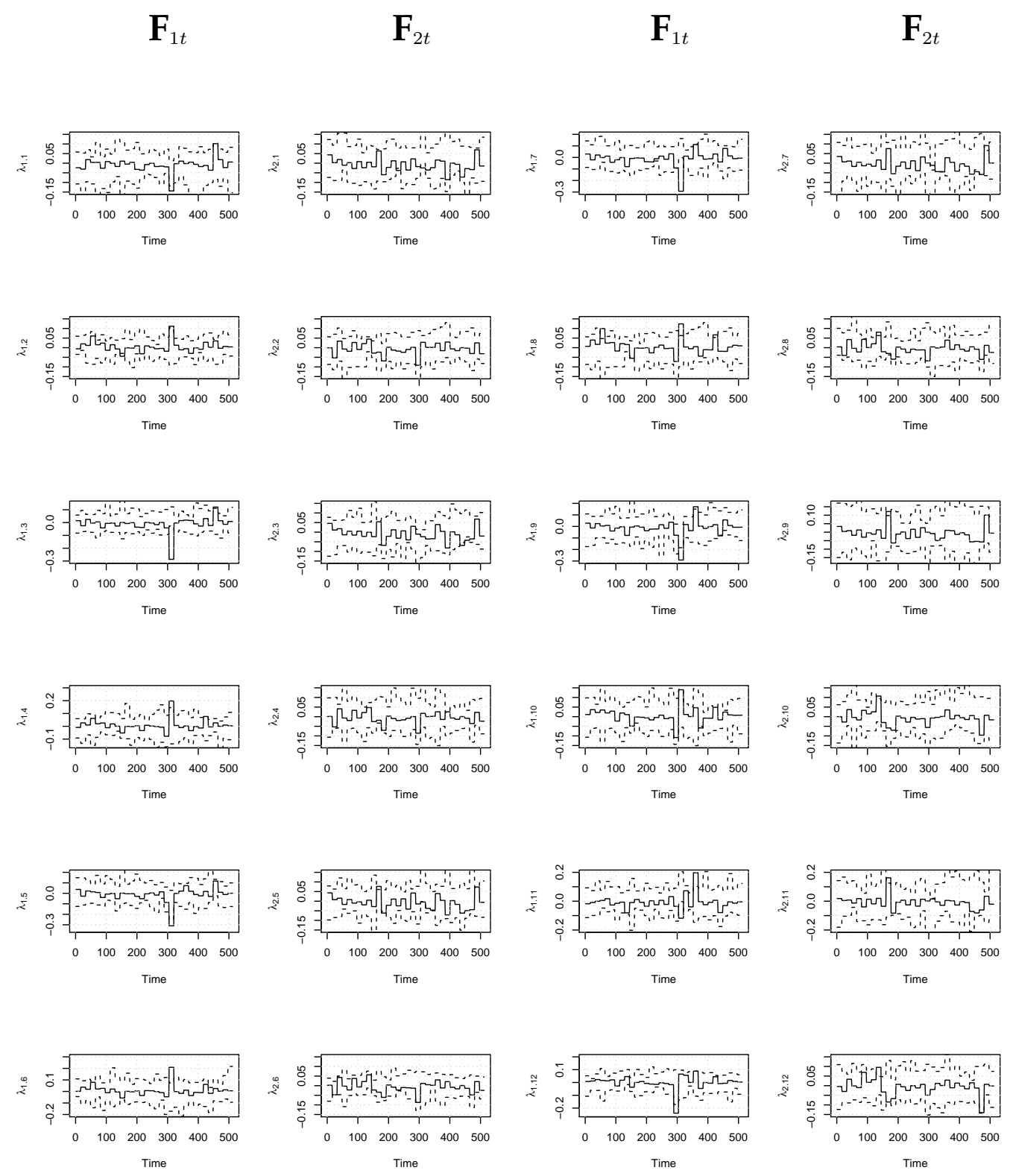
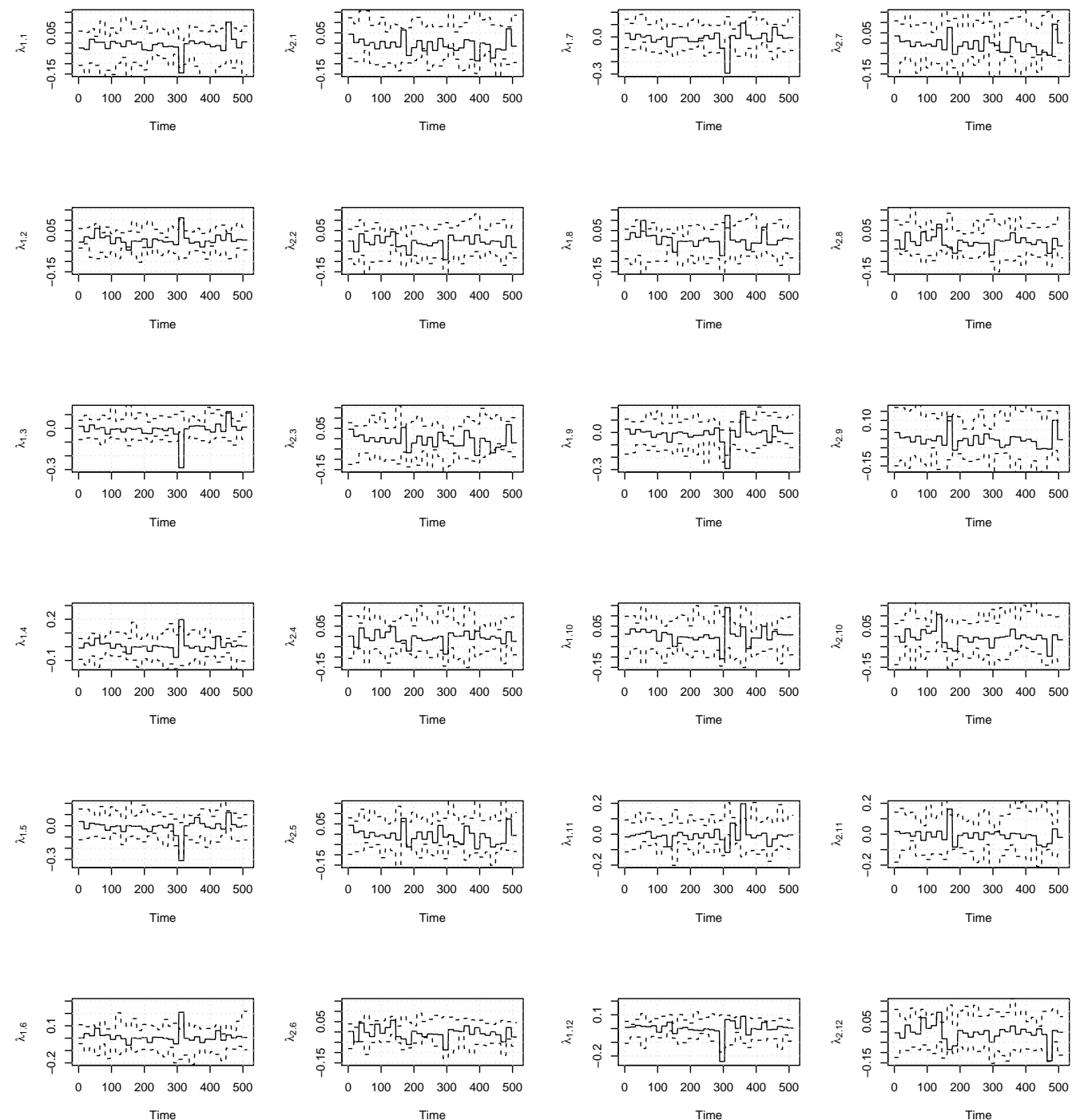

Figura 5.6: Cargas estimadas dos fatores $\tilde{\boldsymbol{F}}_{1 t}$ e $\tilde{\boldsymbol{F}}_{2 t}$ das séries temporais $H 1-H 12$, com um intervalo bootstrap de um erro padrão. Neste caso foi empregada a ondaleta Haar. 


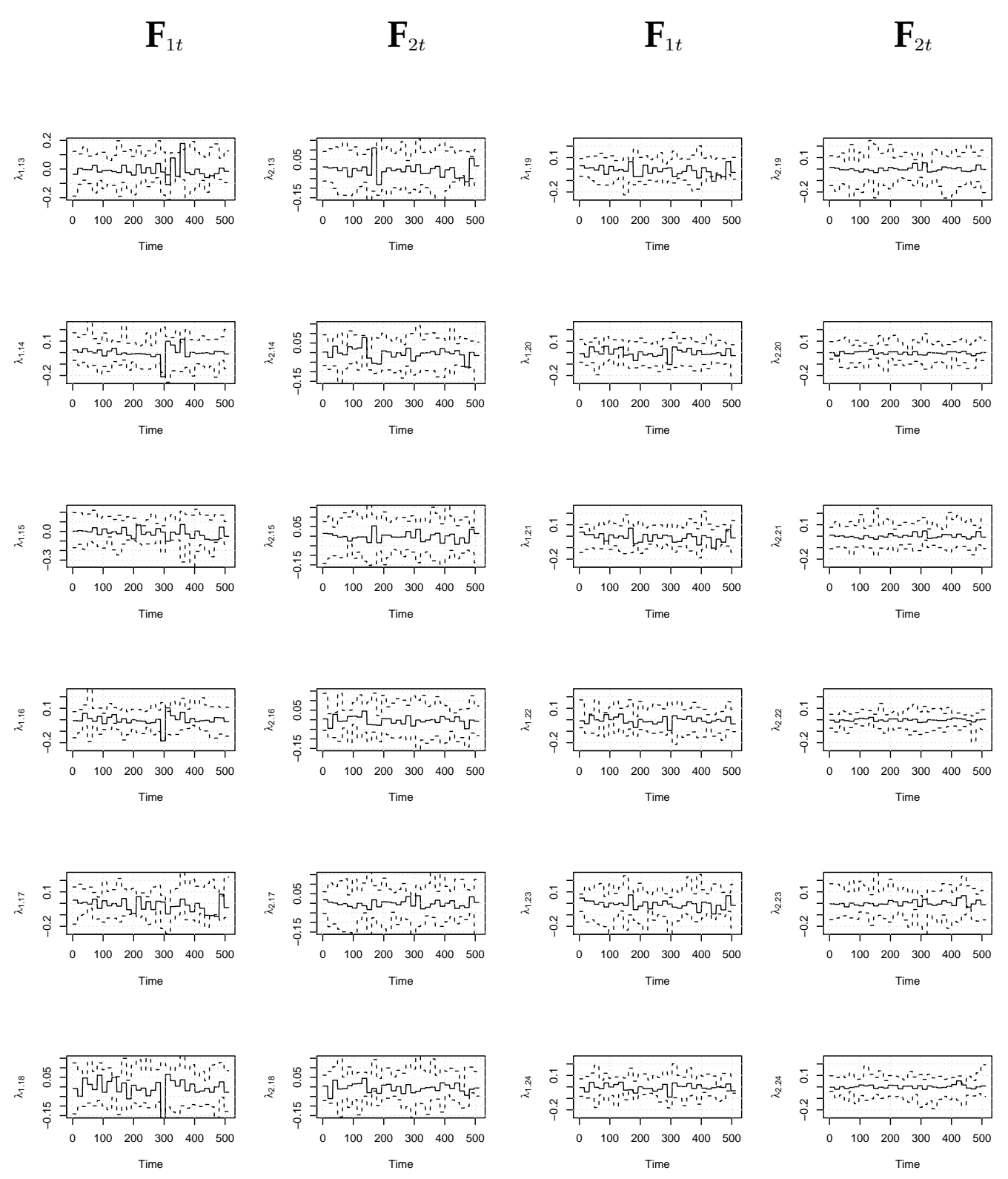

Figura 5.7: Cargas estimadas dos fatores $\tilde{\boldsymbol{F}}_{1 t}$ e $\tilde{\boldsymbol{F}}_{2 t}$ das séries temporais $H 13-H 24$, com um intervalo bootstrap de um erro padrão. Neste caso foi empregada a ondaleta Haar. 


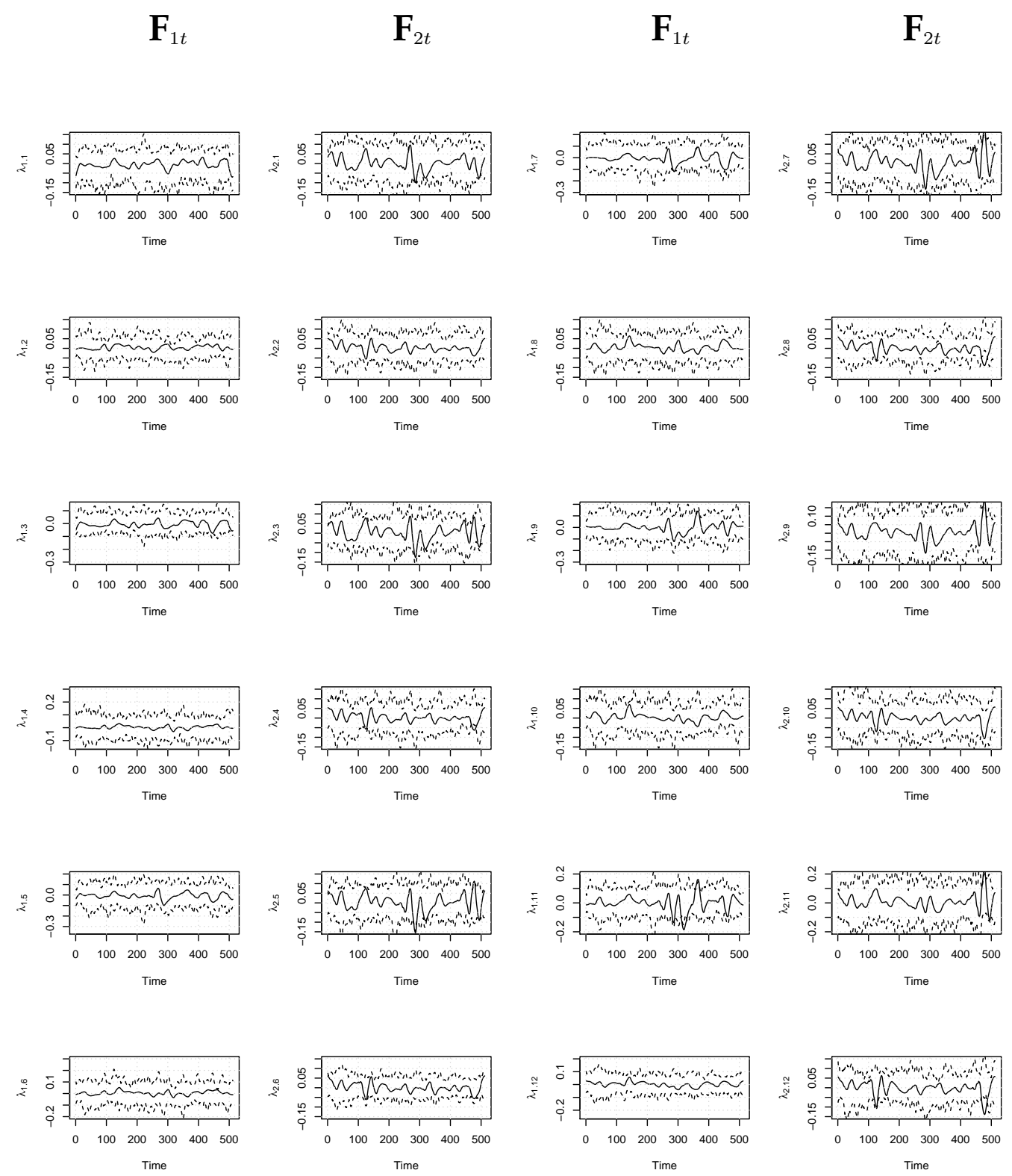
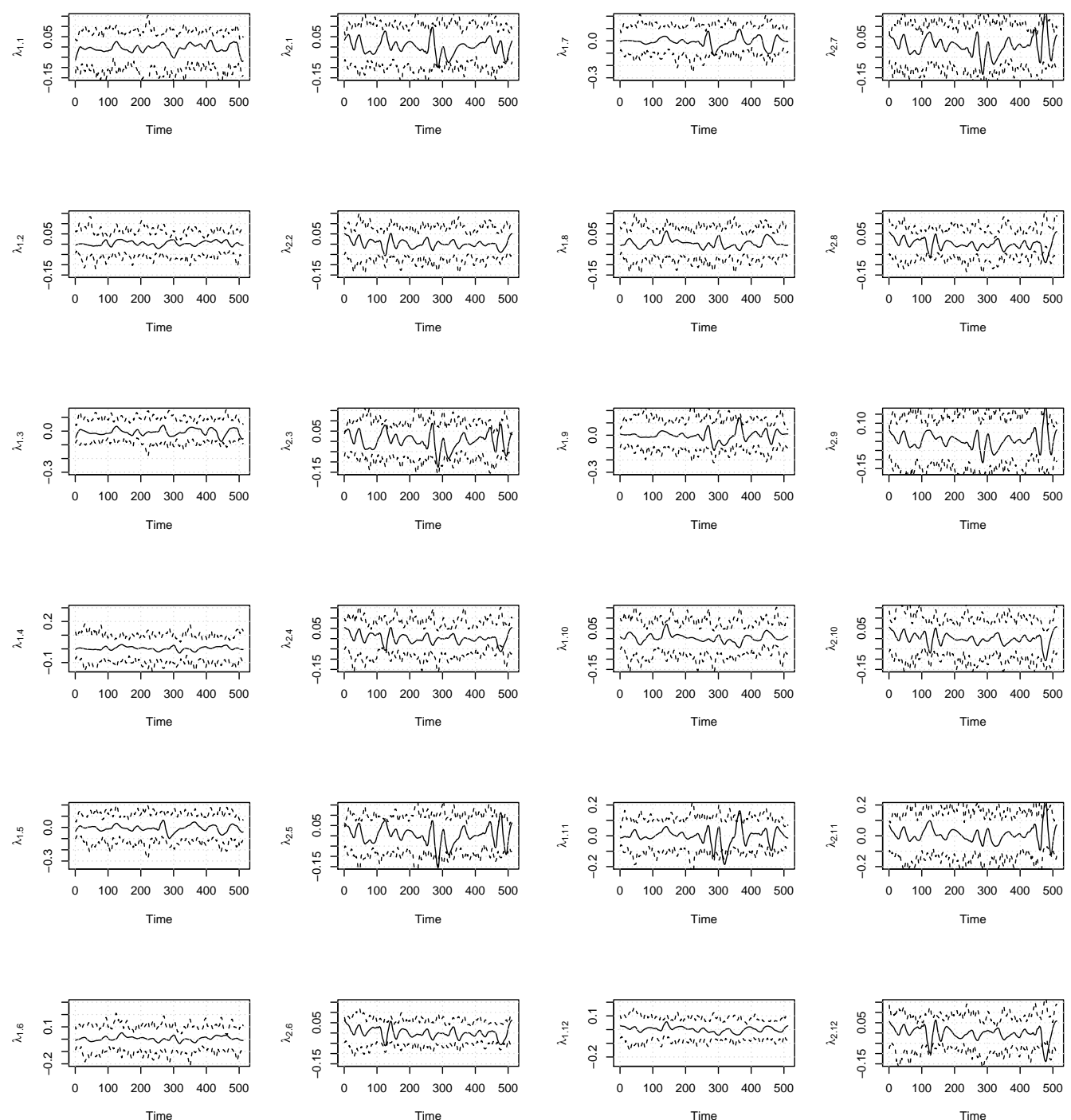

Figura 5.8: Cargas estimadas dos fatores $\tilde{\boldsymbol{F}}_{1 t}$ e $\tilde{\boldsymbol{F}}_{2 t}$ das séries temporais $H 1-H 12$, com um intervalo bootstrap de um erro padrão. Neste caso foi empregada a ondaleta D8. 


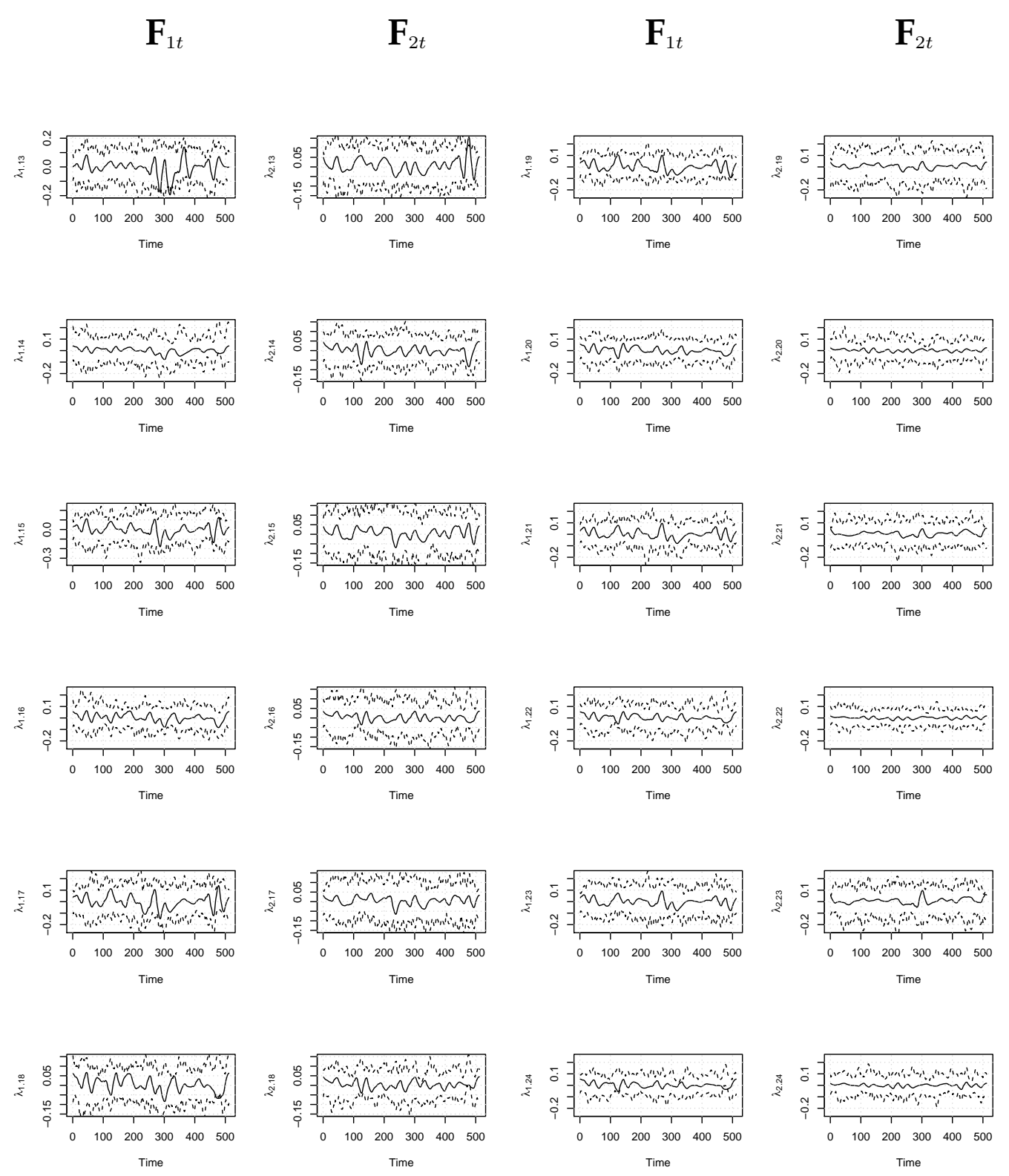

Figura 5.9: Cargas estimadas dos fatores $\tilde{\boldsymbol{F}}_{1 t}$ e $\tilde{\boldsymbol{F}}_{2 t}$ das séries temporais $H 13-H 24$, com um intervalo bootstrap de um erro padrão. Neste caso foi empregada a ondaleta D8. 
Para comparar alguns casos, são apresentamos os gráficos de $\hat{y}_{i t}$ e $y_{i t}$ somente para $i=1, \ldots, 6$. Na Figura 5.10 usamos ondaletas D8 e na Figura 5.11 usamos ondaletas Haar.
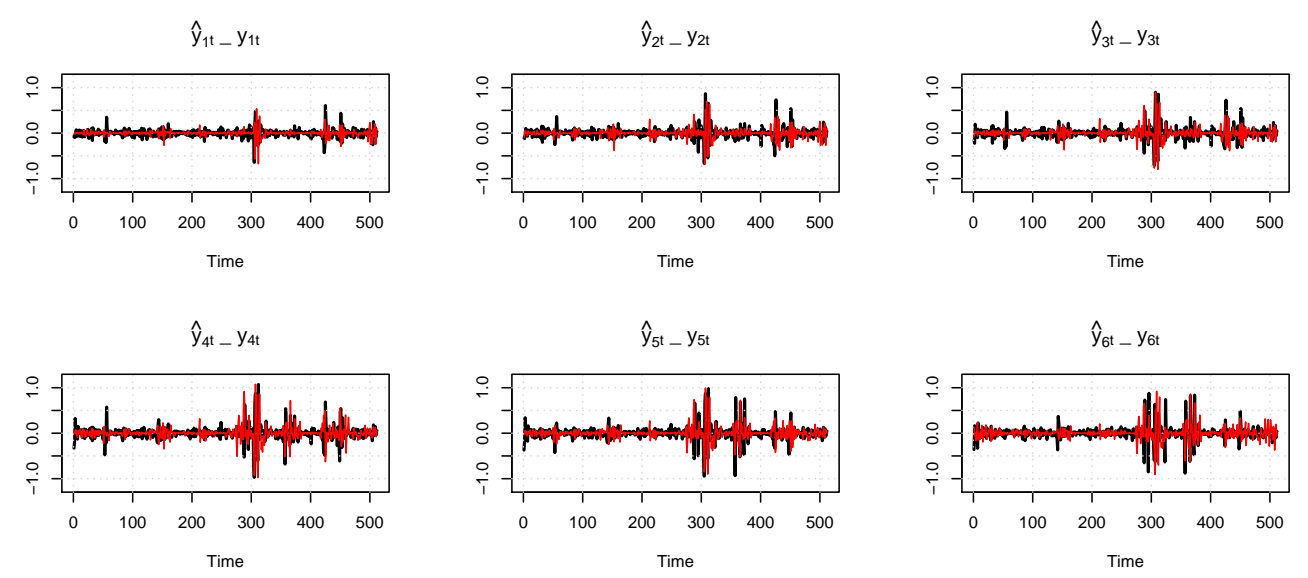

Figura 5.10: Curva estimada $\hat{y}_{i t}$ (vermelho) e curva verdadeira $y_{i t}$ (preto) empregando ondaleta D8.
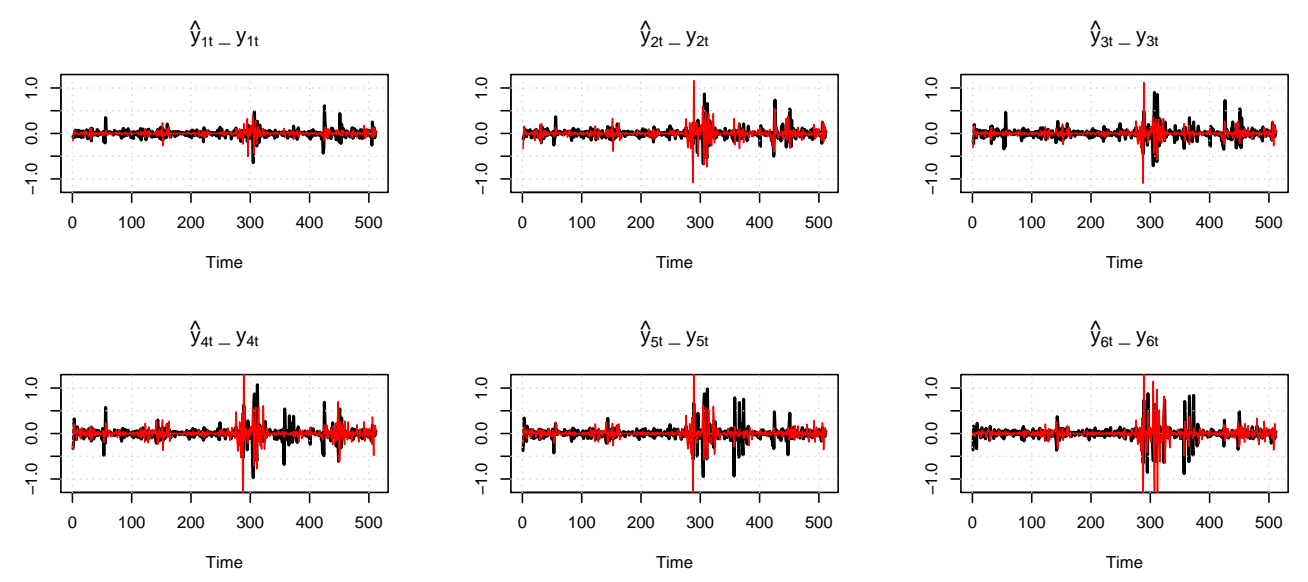

Figura 5.11: Curva estimada $\hat{y}_{i t}$ (vermelho) e curva verdadeira $y_{i t}$ (preto) empregando ondaleta Haar.

\subsection{Conclusões}

Com respeito aos fatores, podemos dizer que para este tipo de séries multivariadas que apresentam co-movimentos em sua estrutura de covariância, eles conseguiram capturar a estrutura comum geral das séries, tanto na parte suave quanto na parte volátil. Enquanto às cargas estimadas, notemos que elas apresentam um valor significativo em aqueles períodos nos quais as séries mostram agrupamentos, e oscilam em torno zero naqueles tempos em que as séries apresentam estacionaridade. Observamos que as cargas estimadas empregando a ondaleta D8, apresenta maior suavidade em comparação da ondaleta Haar, e cujo efeito pode ser visto quando é comparado os dados reais com os estimados mostrados nas Figuras 5.10 e 5.11. 


\section{Capítulo 6}

\section{Conclusões e Trabalhos Futuros}

Sugerimos um procedimento iterativo de mínimos quadrados generalizados baseados em funções de ondaletas em dois estágios para modelos fatoriais com cargas funcionais de alta dimensão. As cargas fatoriais variando no tempo são estimadas empregando os coeficientes de ondaletas obtidos através de um modelo regressão em que a matriz de planejamento depende dos fatores comuns estimados através dos componentes principais dos datos.

O desempenho da metodologia proposta considerando funções suaves nas cargas foi avaliado com estudos de simulação. O bom desempenho é demonstrado mesmo com $N$ e $T$ pequenos. Obtemos as propriedades assintóticas do estimador $\hat{\boldsymbol{\beta}}$ definido em (3.18), que é um estimador não viciado e consistente com distribução normal multivariada e provamos que é possível estimar consistentemente uma rotação dos fatores comuns através dos componentes principais.

Ressaltamos que neste trabalho, independência dos fatores latentes e função suave e contínua são requisitos para garantir o bom desempenho dos estimadores propostos.

Foi apresentada a relevância empírica da estrutura de carga variável no tempo usando um conjunto de dados por hora durante um período de 513 dias de preços da energia do Nord Pool. Para a maioria das variáveis, encontramos evidência de variância mudando no tempo empregando o teste de Hoffman e Pagan, em que posteriormente foram estimadas as cargas funcionais evidenciando uma mudança significativa em aqueles períodos nos quais existem co-movimentos.

Como projetos futuros, consideramos relevante explorar a incorporação de diferentes estruturas dinâmicas tanto nos erros idiossincráticos quanto nos fatores. Por exemplo, considerar uma dinâmica DVAR nos fatores, a fim de obter equações de previsão a partir deles. Também propor critérios para o número de fatores supondo esse tipo de cargas variando no tempo, implementação de medidas de diagnóstico, critérios de seleção de modelos e qualidade do ajuste explorando os resíduos. 


\section{Referências Bibliográficas}

[1] Alonso, A. M., García-Martos, C., Rodríguez, J., and Jesús Sánchez, M. (2011). Seasonal dynamic factor analysis and bootstrap inference: application to electricity market forecasting. Technometrics, 53(2),137-151.

[2] Andrews, D. (1988). Laws of large number for dependent non-identically distribution random variables. Econometric Theory, 4, 458-467.

[3] Bai, J. and Ng, S. (2013). Principal components estimation and identification of static factors. Journal of Econometrics 176(1), 18-29.

[4] Bai, J., Ng, S. (2008). Large dimensional factor analysis. Foundations and Trends in Econometrics, 138163.

[5] Bai, J. and Ng, S. (2002). Determining the number of factors in approximate factor models. Econometrica, 70(1), 191-221.

[6] Bates, B., Plagborg-Møller, M., Stock, J. and Watson, M. (2013). Consistent factor estimation in dynamic factor models with structural instability. Journal of Econometrics, 177, 289-304.

[7] Billingsley, P. (1995). Probability and Measure. John Wiley and Sons, University of Chicago.

[8] Breitung, J. and Eickmeier, S. (2011). Testing for structural breaks in dynamic factor models. Journal of Econometrics, 163 (1), 71-84.

[9] Chamberlain, G. and Rothschild, M. (1983). Arbitrage, factor structure, and mean-variance analysis on large asset markets. Econometrica, 51 (5), 1281-1304.

[10] Cohen, A. and Ryan, R. (1995). Wavelets and multiscale signal processing. Chapman $\mathcal{E}$ Hall

[11] Correal M. and Peña, D. (2008). Modelo factorial dinámico threshold. Revista Colombiana de Estadística, 31, 183-192.

[12] Dahlhaus, R. (2000). A likelihood approximation for locally stationary processes. The Annals of Statistics, 28, 1762-1794. 
[13] Dahlhaus, R., Eichler, M. and Sandkuhler J. (1999). Identification of synaptic connections in neural ensembles by graphical models. J Neurosci Methods, 77(1), 93-107.

[14] Dahlhaus, R. (1997). Fitting time series models to nonstationary processes. The Annals of Statistics, 25,1-37.

[15] Dahlhaus, R. (1996a). On the kullback-leibler information divergence of locally stationary processes. Stochastic Processes and their Applications, 62, 139-168.

[16] Dahlhaus, R. (1996c). Asymptotic statistical inference for nonstationary processes with evolutionary spectra. In P. M. Robinson and M. Rosenblatt (eds), Athens Conference on Applied Probability and Time Series Analysis, Vol. II, Springer-Verlag, New York.

[17] Daubechies, I. (1992). Ten Lectures on Wavelets. Philadelphia. SIAM.

[18] Donoho, D.L. and Johnstone, I. M. (1994). Ideal spatial adaptation by wavelet shrinkage. Biometrika, 81, 425-55.

[19] Dordonnat, V., Koopman, S. J., and Ooms, M. (2012). Dynamic factors in periodic time-varying regressions with an application to hourly electricity load modelling. Computational Statistics $\mathcal{E}$ Data Analysis, 56(11), 3134-3152.

[20] Eichler, M., Motta, G. and von Sachs, R. (2011). Fitting dynamic factor models to nonstationary time series. Journal of Econometrics, 163, 51-70.

[21] Forni, M., Hallin, M., Lippi, M. and Reichlin, L. (2000). The generalized dynamic factor model: identification and estimation. Rev. Econ. Statist., 82, 540-554.

[22] Geweke, J. (1977). The Dynamic Factor Analysis of Economic Time Series, in D. J. Aigner and A. S. Goldberger, eds, "Latent Variables in Socio-Economic Models", North-Holland.

[23] Grossmann, A. and Morlet, J. (1985). Decomposition of Functions into wavelets of constant shape, and related transforms. In: STREIT, L. (ed.). Mathematics + Physics, Lectures on Recent Results. Singapore, World Scientific, 135-165.

[24] Han, X. and Inoue, A. (2015). Tests for Parameter Instability in Dynamic Factor Models. Econometric Theory, 31, 1117-1152.

[25] Hansen, B. (2001). The New Econometrics of Structural Change: Dating Breaks in U.S. Labour Productivity. Journal of Economic Perspectives, 15(4),117-128.

[26] Hoffman, D. and Pagan, A. R. (1989). Post-sample prediction tests for generalized method of moment estimators. Oxford Bulletin of Economics and Statistics, 51, 333-343.

[27] Johnson, R.A. and Wichern, D.W. (2007) Applied Multivariate Statistical Analysis. 6th Edition, Pearson Prentice Hall, Upper Saddle River. 
[28] Kim, C. (1999). Econometric analysis of evolutionary time series. Technical report, Yale University \& Humboldt-Universität zu Berlin.

[29] Lam, C. and Yao, Q. (2012). Factor modeling for high-dimensional time series: inference for the number of factors. Annals of Statistics, 40, 694-726.

[30] Lam, C., Yao, Q. and Bathia, N. (2011). Estimation of latent factors for high-dimensional time series. Biometrika, 98, 901-918.

[31] Lütkepohl, H. and Poskitt, D. (1996). Testing for Causation Using Infinite Order Vector Autoregressive Processes. EconPapers, 12, 61-67.

[32] Mallat, S. (1989). A theory for multiresolution signal decomposition: wavelet representation. IEEE Trans Pattern Anal Mach Intellig, 11, 674-693.

[33] Mallat S. (2008). A Wavelet Tour of Signal Processing: The Sparse Way, ELSEVIER.

[34] Mikkelsen, J., Hillebrand, E. and Urga, G. (2016). Maximum Likelihood Estimation of Time-Varying Loading in High-Dimensional Factor Models. CREATES RP 2017- 22.

[35] Morettin, P. (2014). Ondas e Ondaletas: Da Análise de Fourier à Análise de Ondaletas de Séries Temporais. Edusp - Editora da Universidade de São Paulo, São Paulo.

[36] Motta, G., Hafner, C.M. and von Sachs, R. (2011). Locally stationary factor models: identification and nonparametric estimation. Econometric Theory, 27, 1279 - 1319.

[37] NordPool (2015). Nordpool webpage. http:/ / www.nordpool.com.

[38] Ombao, H. and Ho, M. (2006) Time-dependent frequency domain principal components analysis of multichannel non-stationary signals. Computational and Data Analysis, 50, 2339-2360.

[39] Peña, D. and Box, G. (1987). Identifying a simplifying structure in time series. Journal of the American Statistical Association, 82, 836-843.

[40] Peña, D., Poncela, P., (2006). Nonstationary dynamic factor analysis. Journal of Statistical Planning and Inference 136 (2006) 1237 - 1257

[41] Priestley, M. B. (1981). Spectral Analysis and Time Series. Academic Press, London.

[42] Raviv, E., Bouwman, K. E., and van Dijk, D. (2015). Forecasting day-ahead electricity prices: Utilizing hourly prices. Energy Economics, 50, 227-239.

[43] Rodríguez-Poo, J. M. and Linton, O. (2001). Nonparametric factor analysis of residual time series. Test, 10, 161-182.

[44] Sato, J., Morettin, P.A ., Arantes, P.R. and Amaro Jr, E. (2007) Wavelet based time-varying vector autoregressive modelling. Computational Statistics \& Data Analysis, 51, 5847 - 5866. 
[45] Stock, J.H. and Watson, M.W. (1988). Testing for common trends. Journal of the American Statistical Association, 83, 1097-1107.

[46] Stock, J.H. and Watson, M.W. (2009). Forecasting in Dynamic Factor Models Subject to Structural Instability. The Methodology and Practice of Econometrics: Festschrift in Honor of D.F. Hendry. Oxford. Oxford University Press.

[47] Weron, R. (2007). Modeling and forecasting electricity loads and prices: A statistical approach, volume 403. John Wiley \& Sons.

[48] White, H. (1984). Asymptotic Theory for Econometricians. New York: Academic Press. 


\section{Apêndice A}

\section{Apêndice}

Apresentamos aqui algumas definições e resultados acerca de otimização de funções matriciais e de teoremas de convergência, que são necessários para o desenvolvimento dos resultados principais. Tem alguns lemas auxiliares os quais não tem prova, mas referências são expostas para sua consulta.

\section{A.1 Definições e lemas auxiliares}

Definição A.1.1. (Norma de Frobenius). Seja $A \in \mathbb{C}^{m \times n}$, então sua norma de Frobenius se define como

$$
\|\boldsymbol{A}\|=\sqrt{\operatorname{tr}\left(\boldsymbol{A}^{*} \boldsymbol{A}\right)}=\left(\sum_{j=1}^{m} \sum_{i=1}^{n}\left|a_{j k}\right|^{2}\right)^{1 / 2} .
$$

Definição A.1.2. (Matriz Toeplitz). Uma matriz $A \in \mathbb{C}^{N \times N}$ é denominada matriz de Toeplitz, se cada diagonal paralela à diagonal principal é constante, isto é

$$
a_{j+s, k+s}=a_{j, k}
$$

para todos os possíveis índices $j, k, s$.

Lema A.1.1. (Desigualdade de Cauchy-Schwarz). Sejam A e B matrizes reais de dimensão $m \times n$. Então

$$
\operatorname{tr}^{2}\left\{\boldsymbol{A}^{\prime} \boldsymbol{B}\right\} \leq \operatorname{tr}\left\{\boldsymbol{A}^{\prime} \boldsymbol{A}\right\} \operatorname{tr}\left\{\boldsymbol{B}^{\prime} \boldsymbol{B}\right\}
$$

Lema A.1.2. Sejam A e B matrizes positivas semi-definidas de dimensão $m \times m$. Então

$$
\operatorname{tr}(\boldsymbol{A B}) \leq \operatorname{tr}(\boldsymbol{A}) \operatorname{tr}(\boldsymbol{B})
$$


Os lemas (A.1.3), (A.1.4) e (A.1.5) são resultados da Seção 4.1.3 de Lütkepohl (1996).

Lema A.1.3. Sejam $\boldsymbol{A}$ e $\boldsymbol{B}$ matrizes positivas definidas de $k \times k$. vé autovalor de $\mathbf{B} \boldsymbol{A}$ se e somente se vé autovalor de $\boldsymbol{B}^{1 / 2} A \boldsymbol{B}^{1 / 2}$.

Lema A.1.4. Sejam $\boldsymbol{Y}_{T \times N}$ e $\lambda_{1} \geq \ldots \geq \lambda_{N}$ autovalores de $\boldsymbol{Y}^{\prime} \boldsymbol{Y}$ com autovetores ortonormais $N \times 1$ correspondentes $v_{1}, \ldots, v_{N}$. Com $r(r \leq N)$ autovalores distintos, temos

$$
\min _{\boldsymbol{F}, L}\left\{\operatorname{tr}\left(\boldsymbol{Y}-\boldsymbol{F} \boldsymbol{L}^{\prime}\right)^{\prime}\left(\boldsymbol{Y}-\boldsymbol{F} \boldsymbol{L}^{\prime}\right): \boldsymbol{L}^{\prime} \boldsymbol{L}=\mathbb{I}_{r}\right\}=\sum_{k=1}^{r} \lambda_{k}
$$

e as matrizes que minimizam (A.3) são:

$$
\hat{\boldsymbol{L}}=\left[v_{1}, \ldots, v_{r}\right], \quad \hat{\boldsymbol{F}}=\mathbf{Y} \hat{\mathbf{L}}
$$

Lema A.1.5. Seja $\boldsymbol{Y}_{T \times N}, \boldsymbol{L}_{N \times r} \operatorname{com} \rho(\boldsymbol{L})=r, e \boldsymbol{W}_{T \times T}$ positiva definida. Então

$$
\min _{\boldsymbol{A}}\left\{\operatorname{tr}\left(\boldsymbol{Y}-\boldsymbol{A} \boldsymbol{L}^{\prime}\right)^{\prime} \boldsymbol{W}\left(\boldsymbol{Y}-\boldsymbol{A} \boldsymbol{L}^{\prime}\right): \boldsymbol{A}_{T \times r}\right\}=\operatorname{tr}\left\{\boldsymbol{W} \boldsymbol{Y} \boldsymbol{Y}^{\prime}-\boldsymbol{W} \boldsymbol{Y} \boldsymbol{L}\left(\boldsymbol{L}^{\prime} \boldsymbol{L}\right)^{-1} \mathbf{L}^{\prime} \mathbf{Y}^{\prime}\right\}
$$

O mínimo é atingido quando $A=\mathbf{Y}\left(\boldsymbol{L}^{\prime} \boldsymbol{L}\right)^{-1}$.

Lema A.1.6. (Desigualdade $c_{r}$ de Loève). (Billingsley,1995). Sejam $\boldsymbol{X}_{1}, \ldots, \boldsymbol{X}_{m}$ vetores aleatórios. Então para $r>0$

$$
\mathbb{E}\left\|\sum_{i=1}^{m} \boldsymbol{X}_{i}\right\|^{r} \leq c_{r} \sum_{i=1}^{m} \mathbb{E}\left\|\boldsymbol{X}_{i}\right\|^{r},
$$

em que $c_{r}=1$ quando $r \leq 1$ e $c_{r}=m^{r-1}$ quando $r>1$.

Definição A.1.3. Sequência de vetores diferenças martingais. Seja $\mathcal{F}_{t}$ a $\sigma$-álgebra contendo todas as informações disponíveis até o instante $t$. Um processo estocástico $k$-dimensional $\boldsymbol{Y}_{t}$ é dito uma sequência de vetores diferenças martingais se $\mathbb{E}\left(\boldsymbol{Y}_{t} \mid \mathcal{F}_{t-1}\right)=0$.

Teorema A.1.1. Teorema central do limite para sequência de vetores diferenças martingais. (White, 1984). Seja $\boldsymbol{Y}_{t}, k$-dimensional, uma sequência de diferenças martingais, com $\overline{\boldsymbol{Y}}_{T}=\frac{1}{T} \sum_{t=1}^{T} \boldsymbol{Y}_{t}$, se

a. $\mathbb{E}\left(\boldsymbol{Y}_{t} \boldsymbol{Y}_{t}^{\prime}\right)=\boldsymbol{\Gamma}_{t}$, matriz positiva definida, com $\frac{1}{T} \sum_{t=1}^{T} \boldsymbol{\Gamma}_{t} \rightarrow \boldsymbol{\Gamma} ;$

b. $\mathbb{E}\left(Y_{i t} Y_{j t} Y_{l t} Y_{m t}\right)<\infty$;

c. $\frac{1}{T} \sum_{t=1}^{T} \boldsymbol{Y}_{t} \boldsymbol{Y}_{t}^{\prime} \stackrel{p}{\rightarrow} \boldsymbol{\Gamma}$.

Então $\frac{1}{T} \sum_{t=1}^{T} \overline{\boldsymbol{Y}}_{T} \stackrel{d}{\rightarrow} \mathcal{N}(0, \boldsymbol{\Gamma})$. 
Definição A.1.4. L $L^{1}$-Mixingais. (Andrews, 1988). Seja uma sequência de variáveis aleatórias $\left\{Y_{t}\right\}_{t=1}^{\infty}$ em que $\mathbb{E}\left(Y_{t}\right)=0$, para $t \in \mathbb{N}$. Considere $\mathcal{F}_{t}$ a $\sigma$-álgebra contendo todas as informações disponíveis até o instante $t$. Se existe sequências não negativas de constantes $\left\{a_{t}\right\}_{t=1}^{\infty} e\left\{\alpha_{t}\right\}_{t=1}^{\infty}$ tal que $\lim _{t \rightarrow \infty} \alpha_{t}=0 e$

$$
\mathbb{E}\left|\mathbb{E}\left(Y_{t} \mid \mathcal{F}_{t-m}\right)\right| \leq a_{t} \alpha_{m},
$$

$\forall t \in \mathbb{N} e \forall m \geq 0$, então $\left\{Y_{t}\right\}_{t=1}^{\infty}$ é definida um $L^{1}$-mixingal com respeito à $\left\{\mathcal{F}_{t}\right\}_{t=1}^{\infty}$.

Teorema A.1.2. Lei fraca dos grandes números para $L^{1}$-mixingais. (Andrews, 1988). Sejam $\{Y\}_{t=1}^{\infty} u m$ $L^{1}$-mixingal da Definião A.1.4. Se $Y_{t}$ é uniformemente integrável e $\lim _{t \rightarrow \infty}(1 / T) \sum_{t=1}^{T} a_{t}<\infty$, então $(1 / T) \sum_{t=1}^{T} Y_{t} \stackrel{p}{\rightarrow} 0$.

\section{A.2 Provas}

Prova Proposição 2.1.2. Utilizando o Teorema de Representação Espectral para o processo estacionário $\mathbf{F}_{t}$ temos

$$
\mathbf{F}_{t-k}=\frac{1}{\sqrt{2 \pi}} \int_{-\pi}^{\pi} e^{i \omega(t-k)} d \boldsymbol{\xi}(\omega)
$$

em que $\boldsymbol{\xi}(\omega)$ é um processo estocástico sobre $[-\pi, \pi]$ com incrementos ortogonais, que satisfaz $(a)$ da Definição 2.1.1. Assim substituindo (A.6) em (2.9) obtemos

$$
\begin{aligned}
\mathbf{X}_{t} & =\sum_{k=0}^{\infty} \mathbf{s}_{k}\left(\frac{t}{T}\right) \mathbf{F}_{t-k} \\
& =\sum_{k=0}^{\infty} \mathbf{s}_{k}\left(\frac{t}{T}\right)\left[(2 \pi)^{-1 / 2} \int_{-\pi}^{\pi} e^{i \omega(t-k)} d \boldsymbol{\xi}(\omega)\right] \\
& =\sum_{k=0}^{\infty} \mathbf{s}_{k}\left(\frac{t}{T}\right)\left[(2 \pi)^{-1 / 2} \int_{-\pi}^{\pi} e^{i \omega t} e^{-i \omega k} d \boldsymbol{\xi}(\omega)\right] \\
& =\int_{-\pi}^{\pi} \underbrace{\left[\sum_{k=0}^{\infty} \mathbf{s}_{k}\left(\frac{t}{T}\right)(2 \pi)^{-1 / 2} e^{-i \omega k}\right]}_{\mathbf{A}_{T}^{0}(t, \omega)} e^{i \omega t} d \boldsymbol{\xi}(\omega) \\
& =\int_{-\pi}^{\pi} \mathbf{A}_{T}^{0}(t, \omega) e^{i \omega t} d \boldsymbol{\xi}(\omega) .
\end{aligned}
$$

Neste caso temos que $\mathbf{A}_{T}^{0}(t, \omega)=\mathbf{A}\left(\frac{t}{T}, \omega\right)$, satisfazendo assim a condição (b) da Definição 2.1.1, mesmo que o processo $\boldsymbol{\xi}(\omega)$ satisfaz $(a)$. 
Lema A.2.1. Sob as suposições S1-S5, quando $N, T \rightarrow \infty$

$$
\left\|V_{N T}-\frac{\tilde{\boldsymbol{F}}^{\prime} \boldsymbol{F}}{T} \frac{\boldsymbol{\Lambda}_{0}^{\prime} \boldsymbol{\Lambda}_{0}}{N} \frac{\boldsymbol{F}^{\prime} \tilde{\boldsymbol{F}}}{T}\right\|^{2}=O_{p}\left(C_{N T}^{-2}\right)
$$

em que $V_{N T}$ é uma matriz diagonal composta dos $r$ maiores autovalores da matriz $(N T)^{-1} \mathbf{Y}^{\prime}$.

Prova. Ver Mikkelsen et al. (2016).

Prova Teorema 3.1.1. Seja $\mathbf{Y}=\left(Y_{1}, \ldots, Y_{T}\right)^{\prime}$ a matriz de $T \times N$ de observações, e seja $V_{N T}$ uma matriz diagonal de dimensão $r \times r$, composta por $r$ autovalores da matriz $(N T)^{-1} \mathbf{Y} \mathbf{Y}^{\prime}$ em ordem decrescente. Pela definição de autovetor e autovalor, temos que

$$
\frac{1}{N T} \mathbf{Y} \mathbf{Y}^{\prime} \tilde{\mathbf{F}}=\tilde{\mathbf{F}} V_{N T} \Longleftrightarrow \frac{1}{N T} \mathbf{Y} \mathbf{Y}^{\prime} \tilde{\mathbf{F}} V_{N T}^{-1}=\tilde{\mathbf{F}}
$$

em que $\tilde{\mathbf{F}}^{\prime} \tilde{\mathbf{F}}=\mathbb{I}_{r}$. O modelo definido em (3.1) e (3.2) pode ser escrito como

$$
\begin{aligned}
\mathbf{Y}_{t} & =\left[\boldsymbol{\Lambda}_{0}+\boldsymbol{\Lambda}(t)\right] \mathbf{F}_{t}+\mathbf{e}_{t} \\
& =\boldsymbol{\Lambda}_{0} \mathbf{F}_{t}+\boldsymbol{\Lambda}(t) \mathbf{F}_{t}+\mathbf{e}_{t} \\
& =\boldsymbol{\Lambda}_{0} \mathbf{F}_{t}+\mathbf{w}_{t}+\mathbf{e}_{t}
\end{aligned}
$$

em que $\mathbf{w}_{t}=\boldsymbol{\Lambda}(t) \mathbf{F}_{t}$. Definamos $\mathbf{e}=\left(\mathbf{e}_{1}, \mathbf{e}_{2}, \ldots, \mathbf{e}_{T}\right)^{\prime}$ e $\mathbf{w}=\left(\mathbf{w}_{1}, \mathbf{w}_{2}, \ldots, \mathbf{w}_{T}\right)^{\prime}$ matrizes de ordens $T \times N$. Notemos que o modelo (3.1), pode ser escrito na forma matricial

$$
\mathbf{Y}=\mathbf{F} \boldsymbol{\Lambda}_{0}^{\prime}+\mathbf{w}+\mathbf{e}
$$

Portanto, ao realizar a multiplicação matricial obtemos

$$
\mathbf{Y} \mathbf{Y}^{\prime}=\mathbf{F} \boldsymbol{\Lambda}_{0}^{\prime} \boldsymbol{\Lambda}_{0} \mathbf{F}^{\prime}+\mathbf{F} \boldsymbol{\Lambda}_{0}^{\prime}(\mathbf{e}+\mathbf{w})^{\prime}+(\mathbf{e}+\mathbf{w}) \boldsymbol{\Lambda}_{0} \mathbf{F}^{\prime}+(\mathbf{e}+\mathbf{w})(\mathbf{e}+\mathbf{w})^{\prime}
$$

A partir da definição de $\tilde{\mathbf{F}}_{t}$ em (A.7) e da matriz de rotação $H$ dada por hipótese, podemos escrever a diferença, para $t$ fixo, como 


$$
\begin{aligned}
\tilde{\mathbf{F}}_{t}-H^{\prime} \mathbf{F}_{t}= & (N T)^{-1} V_{N T}^{-1} \tilde{\mathbf{F}}^{\prime} \mathbf{Y} \mathbf{Y}_{t}^{\prime}-V_{N T}^{-1}\left(\tilde{\mathbf{F}}^{\prime} \mathbf{F} / T\right)\left(\boldsymbol{\Lambda}_{0}^{\prime} \boldsymbol{\Lambda}_{0} / N\right) \mathbf{F}_{t} \\
= & \frac{V_{N T}^{-1}}{N T}\left[\tilde{\mathbf{F}}^{\prime} \mathbf{Y} \mathbf{Y}_{t}^{\prime}-\left(\tilde{\mathbf{F}}^{\prime} \mathbf{F}\right)\left(\boldsymbol{\Lambda}_{0}^{\prime} \boldsymbol{\Lambda}_{0}\right) \mathbf{F}_{t}\right] \\
= & \frac{V_{N T}^{-1}}{N T}\left[\tilde{\mathbf{F}}^{\prime}\left(\mathbf{F} \boldsymbol{\Lambda}_{0}^{\prime} \boldsymbol{\Lambda}_{0} \mathbf{F}_{t}+\mathbf{F} \mathbf{\Lambda}_{0}^{\prime}\left(w_{t}+e_{t}\right)+(w+e) \boldsymbol{\Lambda}_{0} \mathbf{F}_{t}+(e+w)\left(e_{t}+w_{t}\right)\right)\right. \\
& \left.-\left(\tilde{\mathbf{F}}^{\prime} \mathbf{F}\right)\left(\boldsymbol{\Lambda}_{0}^{\prime} \boldsymbol{\Lambda}_{0}\right) \mathbf{F}_{t}\right] \\
= & \frac{V_{N T}^{-1}}{N T}\left[\tilde{\mathbf{F}}^{\prime} \mathbf{F} \boldsymbol{\Lambda}_{0}^{\prime}\left(w_{t}+e_{t}\right)+\tilde{\mathbf{F}}^{\prime}(w+e) \boldsymbol{\Lambda}_{0} \mathbf{F}_{t}+\tilde{\mathbf{F}}^{\prime}(w+e)\left(w_{t}+e_{t}\right)\right] \\
= & \frac{V_{N T}^{-1}}{N T}\left[\tilde{\mathbf{F}}^{\prime} \mathbf{F} \boldsymbol{\Lambda}_{0}^{\prime} e_{t}+\tilde{\mathbf{F}}^{\prime} e \boldsymbol{\Lambda}_{0} \mathbf{F}_{t}+\tilde{\mathbf{F}}^{\prime} e e_{t}+\tilde{\mathbf{F}}^{\prime} \mathbf{F} \boldsymbol{\Lambda}_{0}^{\prime} w_{t}+\tilde{\mathbf{F}}^{\prime} w \boldsymbol{\Lambda}_{0} \mathbf{F}_{t}+\tilde{\mathbf{F}}^{\prime} w w_{t}+\right. \\
& \left.\tilde{\mathbf{F}}^{\prime} e w_{t}+\tilde{\mathbf{F}}^{\prime} w e_{t}\right] .
\end{aligned}
$$

Enumerando cada um dos termos do lado direito da equação como $D_{i t}, i=1, \ldots, 8$, respectivamente, obtemos

$$
\tilde{\mathbf{F}}_{t}-H^{\prime} \mathbf{F}_{t}=V_{N T}^{-1} \sum_{i=1}^{8} D_{i t}
$$

Agora, aplicando norma ao quadrado em ambos lados, somando sob $t$ e dividindo por $T$ na equação (A.11), obtemos pela desigualdade de Loèv

$$
\frac{1}{T} \sum_{t=1}^{T}\left\|\tilde{\mathbf{F}}_{t}-H^{\prime} \mathbf{F}_{t}\right\|^{2} \leq\left\|V_{N T}^{-1}\right\|^{2} 8 \sum_{i=1}^{8}\left(\frac{1}{T} \sum_{t=1}^{T}\left\|D_{i t}\right\|^{2}\right)
$$

em que

$$
\begin{array}{rlrl}
D_{1 t} & =\tilde{\mathbf{F}}^{\prime} \mathbf{F} \boldsymbol{\Lambda}_{0}^{\prime} e_{t} / N T & D_{2 t}=\tilde{\mathbf{F}}^{\prime} e \boldsymbol{\Lambda}_{0} \mathbf{F}_{t} / N T \\
D_{3 t}=\tilde{\mathbf{F}}^{\prime} e e_{t} / N T & D_{4 t}=\tilde{\mathbf{F}}^{\prime} \mathbf{F} \boldsymbol{\Lambda}_{0}^{\prime} w_{t} / N T \\
D_{5 t}=\tilde{\mathbf{F}}^{\prime} w \boldsymbol{\Lambda}_{0} \mathbf{F}_{t} / N T & D_{6 t}=\tilde{\mathbf{F}}^{\prime} w w_{t} / N T \\
D_{7 t}=\tilde{\mathbf{F}}^{\prime} e w_{t} / N T & D_{8 t}=\tilde{\mathbf{F}}^{\prime} w e_{t} / N T
\end{array}
$$

Notemos primeiro que pelo Lema A.2.1 dado que $V_{N T}$ converge a uma matriz positiva definida então temos $\left\|V_{N T}^{-1}\right\|=O_{p}(1)$. Agora vamos analisar cada um dos termos $D_{i t}, \quad i=1, \ldots, 8$.

Para o termo $D_{1 t}$ temos:

$$
\begin{aligned}
\left\|D_{1 t}\right\|^{2} & =\frac{\left\|\tilde{\mathbf{F}}^{\prime} \mathbf{F} \boldsymbol{\Lambda}_{0}^{\prime} e_{t}\right\|^{2}}{N^{2} T^{2}} \\
& =\frac{\left\|\tilde{\mathbf{F}}^{\prime}\right\|^{2}}{T}\left(T^{-1} \sum_{t=1}^{T}\left\|\mathbf{F}_{t}\right\|^{2}\right)\left\|N^{-1} \boldsymbol{\Lambda}_{0}^{\prime} e_{t}\right\|^{2}
\end{aligned}
$$

Notemos que por definição dos $\mathrm{CP}$, para o primeiro termo temos $\|\tilde{\mathbf{F}}\|^{2} / T=\operatorname{tr}[\tilde{\mathbf{F}} \tilde{\mathbf{F}} / T]=\operatorname{tr}\left[\mathbb{I}_{r}\right]=r$. A suposição (S1.b) implica que $\left(T^{-1} \sum_{t=1}^{T}\left\|\mathbf{F}_{t}\right\|^{2}\right)=O_{p}(1)$. Para o último fator 


$$
\begin{aligned}
\mathbb{E}\left\|\frac{\Lambda_{0}^{\prime} e_{t}}{N}\right\|^{2} & \leq \frac{1}{N^{2}} \sum_{i=1}^{N} \sum_{j=1}^{N}\left|\mathbb{E}\left(e_{i t} e_{j t}\right) \lambda_{i 0}^{\prime} \lambda_{j 0}\right| \\
& \leq N^{-1} \bar{\lambda}^{2}\left(N^{-1} \sum_{i=1}^{N} \sum_{j=1}^{N}\left|\mathbb{E}\left(e_{i t} e_{j t}\right)\right|\right) \\
& \leq N^{-1} \bar{\lambda}^{2} Q, \quad(S 3 . b) .
\end{aligned}
$$

Somando sob $t$ e dividindo por $T$, obtemos

$$
\begin{aligned}
T^{-1} \sum_{t=1}^{T}\left\|D_{1 t}\right\|^{2} & \leq \frac{1}{T} \sum_{t=1}^{T} r O_{p}(1) \frac{1}{N} \bar{\lambda}^{2} Q \\
& =\frac{r O_{p}(1) \bar{\lambda}^{2} Q}{N} .
\end{aligned}
$$

Assim obtemos $T^{-1} \sum_{t=1}^{T} \mathbb{E}\left\|D_{1 t}\right\|^{2}=O_{p}\left(N^{-1}\right)$.

Para $D_{2 t}$ obtemos:

$$
\begin{aligned}
\left\|D_{2 t}\right\|^{2} & =\frac{\left\|\tilde{\mathbf{F}}^{\prime} e \boldsymbol{\Lambda}_{0} \mathbf{F}_{t}\right\|^{2}}{N^{2} T^{2}} \\
& \leq\left(T^{-1} \sum_{t=1}^{T}\left\|\tilde{\mathbf{F}}_{t}\right\|^{2}\right)\left(T^{-1} \frac{\left\|e \boldsymbol{\Lambda}_{0} \mathbf{F}_{t}\right\|^{2}}{N^{2}}\right)
\end{aligned}
$$

Novamente pela propriedade dos CP o primeiro termo é limitado por $\|\tilde{\mathbf{F}}\|^{2} / T=\operatorname{tr}\left[\tilde{\mathbf{F}}^{\prime} \tilde{\mathbf{F}} / T\right]=\operatorname{tr}\left[\mathbb{I}_{r}\right]=r$. Para o segundo fator

$$
\begin{aligned}
\mathbb{E}\left(\frac{\left\|e \boldsymbol{\Lambda}_{\mathbf{0}} \mathbf{F}_{t}\right\|^{2}}{N^{2} T}\right) & \leq N^{-1} \sum_{i=1}^{N} \sum_{j=1}^{N}\left|\mathbb{E}\left[e_{i t} e_{j t} / N\right] \lambda_{i 0} \lambda_{j 0} \mathbb{E}\left[\left\|\mathbf{F}_{t}\right\|^{2} / T\right]\right| \\
& \leq N^{-1} \bar{\lambda}^{2} \sum_{i=1}^{N} \sum_{j=1}^{N}\left|\mathbb{E}\left[e_{i t} e_{j t} / N\right] \mathbb{E}\left[\left\|\mathbf{F}_{t}\right\|^{2} / T\right]\right| \\
& \leq N^{-1} \bar{\lambda}^{2} Q \mathbb{E}\left[\left\|\mathbf{F}_{t}\right\|^{2} / T\right]
\end{aligned}
$$

Assim somando sob $t$ e dividindo entre $T$

$$
T^{-1} \sum_{t=1}^{T}\left\|D_{2 t}\right\|^{2} \leq r(N T)^{-1} \bar{\lambda}^{2} Q \mathbb{E}\left[\sum_{t=1}^{T}\left\|\mathbf{F}_{t}\right\|^{2} / T\right],
$$

da suposição (S1.b) temos $T^{-1} \sum_{t=1}^{T}\left\|D_{2 t}\right\|^{2}=O_{p}\left(N^{-1} T^{-1}\right)$.

Considerando o termo $D_{3 t}$ temos:

$$
\begin{aligned}
\left\|D_{3 t}\right\|^{2} & =\frac{\left\|\tilde{\mathbf{F}}^{\prime} e e_{t}\right\|^{2}}{N^{2} T^{2}} \\
& \leq\left(N^{2} T\right)^{-1}\left(\frac{1}{T} \sum_{s=1}^{T}\left\|\tilde{\mathbf{F}}_{s}\right\|^{2}\right)\left\|e e_{t}\right\|^{2} \\
& \leq(N T)^{-1} r \sum_{i=1}^{N} \sum_{j=1}^{N} \mathbb{E}\left(e_{i t} e_{j t} / N\right) \\
& \leq(N T)^{-1} r Q, \quad(S 3 . b) .
\end{aligned}
$$

Portanto $T^{-1} \sum_{t=1}^{T}\left\|D_{3 t}\right\|^{2}=O_{p}\left(N^{-1} T^{-1}\right)$.

Para o quarto termo 


$$
\begin{aligned}
\left\|D_{4 t}\right\|^{2} & =\left\|\tilde{\mathbf{F}}^{\prime} \mathbf{F} \boldsymbol{\Lambda}_{0}^{\prime} w_{t}\right\|^{2}(N T)^{-2} \\
& \leq \frac{\left\|\tilde{\mathbf{F}}^{\prime}\right\|^{2}}{T} \frac{\|\mathbf{F}\|^{2}}{T} \frac{\left\|\boldsymbol{\Lambda}_{0}^{\prime} w_{t}\right\|^{2}}{N^{2}} \\
& \leq\left(T^{-1} \sum_{s=1}^{T}\left\|\tilde{\mathbf{F}}_{s}\right\|^{2}\right)\left(T^{-1} \sum_{t=1}^{T}\left\|\mathbf{F}_{t}\right\|^{2}\right)\left\|N^{-1} \boldsymbol{\Lambda}_{0}^{\prime} w_{t}\right\|^{2}
\end{aligned}
$$

Os dois primeiros fatores são similares aos correspondentes do termo $D_{1 t}$. Para o terceiro fator

$$
\begin{aligned}
\mathbb{E}\left\|\frac{\boldsymbol{\Lambda}_{0}^{\prime} w_{t}}{N}\right\|^{2} & \leq \frac{1}{N^{2}} \sum_{i=1}^{N} \sum_{j=1}^{N}\left|\mathbb{E}\left(w_{i t} w_{j t}\right) \lambda_{i 0}^{\prime} \lambda_{j 0}\right| \\
& \leq N^{-2} \bar{\lambda}^{2} \sum_{i=1}^{N} \sum_{j=1}^{N}\left|\mathbb{E}\left[\lambda_{i t}^{\prime} \mathbf{F}_{t} \lambda_{j t}^{\prime} \mathbf{F}_{t}\right]\right|, \quad(S 2 . b) \\
& \leq r^{2} \bar{\lambda}^{2} \sup _{p, q} N^{-2} \sum_{i=1}^{N} \sum_{j=1}^{N}\left|\mathbb{E}\left[\lambda_{i p, t} F_{t p} \lambda_{j q, t} F_{t q}\right]\right| \\
& \leq r^{2} \bar{\lambda}^{2} N^{-2} K_{1 N T}, \quad(S 4 . a) .
\end{aligned}
$$

Como consequência, uniformemente em $t$, obtemos

$$
T^{-1} \sum_{t=1}^{T}\left\|D_{4 t}\right\|^{2}=O_{p}\left(N^{-2} K_{1 N T}\right)
$$

Para o termo $D_{5 t}$

$$
\begin{aligned}
\left\|D_{5 t}\right\|^{2} & =\left\|\tilde{\mathbf{F}}^{\prime} w \boldsymbol{\Lambda}_{0} \mathbf{F}_{t}\right\|^{2}(N T)^{-2} \\
& \leq \frac{\left\|\tilde{\mathbf{F}}^{\prime}\right\|^{2}}{T} \frac{\left\|w \boldsymbol{\Lambda}_{0} \mathbf{F}_{t}\right\|^{2}}{N^{2} T} \\
& \leq\left(T^{-1} \sum_{t=1}^{T}\left\|\tilde{\mathbf{F}}_{t}\right\|^{2}\right)\left(\left(N^{2} T\right)^{-1} \sum_{s=1}^{T}\left\|w_{s}^{\prime} \boldsymbol{\Lambda}_{0} \mathbf{F}_{t}\right\|^{2}\right)
\end{aligned}
$$

O primeiro termo é similar aos anteriores devido aos CP. O segundo termo

$$
\begin{aligned}
\left(N^{2} T\right)^{-1} \mathbb{E}\left[\sum_{s=1}^{T}\left(w_{s}^{\prime} \boldsymbol{\Lambda}_{0} \mathbf{F}_{t}\right)^{2}\right] & \leq\left(N^{2} T\right)^{-1} \mathbb{E}\left[\sum_{s=1}^{T} \sum_{i=1}^{N} \sum_{j=1}^{N} w_{i s} w_{j s} \lambda_{0 i}^{\prime} \mathbf{F}_{t} \lambda_{0 j}^{\prime} \mathbf{F}_{t}\right] \\
& \leq \bar{\lambda}^{2}\left(N^{2} T\right)^{-1} \sup _{p, q, n, m} \mathbb{E}\left[\sum_{s=1}^{T} \sum_{i=1}^{N} \sum_{j=1}^{N} \lambda_{i p, s} \lambda_{j q, s} F_{p s} F_{q s} F_{n t} F_{m t}\right] .
\end{aligned}
$$

Então somando sobre $t$ e dividendo por $T$, obtemos $T^{-1} \sum_{t=1}^{T}\left\|D_{5 t}\right\|^{2} \leq r^{4} \bar{\lambda}^{2}\left(N^{2} T\right)^{-1} K_{2 N T}$, por suposição (S4.b). Consequentemente

$$
T^{-1} \sum_{t=1}^{T}\left\|D_{5 t}\right\|^{2}=O_{p}\left(N^{-2} T^{-2} K_{2 N T}\right)
$$

Considerando o termo $D_{6 t}$ 


$$
\begin{aligned}
\left\|D_{6 t}\right\|^{2} & =\left\|\tilde{\mathbf{F}}^{\prime} w w_{t}\right\|^{2}(N T)^{-2} \\
& =\left(N^{2} T\right)^{-1} \frac{\left\|\tilde{\mathbf{F}}^{\prime}\right\|^{2}}{T}\left\|w w_{t}\right\|^{2} . \\
\mathbb{E}\left[\left\|D_{6 t}\right\|^{2}\right] & \leq\left(N^{2} T\right)^{-1}\left(T^{-1} \sum_{t=1}^{T}\left\|\tilde{\mathbf{F}}_{t}\right\|^{2}\right)\left(\sum_{s=1}^{T} \mathbb{E}\left(w_{s}^{\prime} w_{t}\right)^{2}\right) \\
& \leq\left(N^{2} T\right)^{-1} r \sup _{p, q, n, m} \mathbb{E}\left[\sum_{s=1}^{T} \sum_{i=1}^{N} \sum_{j=1}^{N} \lambda_{i p, s} \lambda_{j q, s} \lambda_{i p, t} \lambda_{j q, t} F_{p s} F_{q s} F_{n t} F_{m t}\right] \\
& \leq r^{5}\left(N^{2} T\right)^{-1} K_{3 N T}, \quad(S 4 . c) .
\end{aligned}
$$

Portanto somando sobre $t$ e dividendo por $T$, obtemos

$$
T^{-1} \sum_{t=1}^{T}\left\|D_{6 t}\right\|^{2}=O_{p}\left(N^{-2} T^{-2} K_{3 N T}\right) .
$$

Para $D_{7 t}$ temos

$$
\begin{aligned}
\left\|D_{7 t}\right\|^{2} & =(N T)^{-2}\left\|\tilde{\mathbf{F}}^{\prime} e w_{t}\right\|^{2} \\
& =\left(N^{2} T\right)^{-1} \frac{\left\|\tilde{\mathbf{F}}^{\prime}\right\|^{2}}{T}\left\|e w_{t}\right\|^{2} \\
& =\left(N^{2} T\right)^{-1} r\left\|e w_{t}\right\|^{2} .
\end{aligned}
$$

Agora aplicando valor esperado, obtemos

$$
\begin{aligned}
\mathbb{E}\left[\left\|D_{7 t}\right\|^{2}\right] & =r\left(N^{2} T\right)^{-1} \mathbb{E}\left\|e^{\prime} w_{t}\right\|^{2} \\
& \leq r\left(N^{2} T\right)^{-1} \mathbb{E}\left[\sum_{s=1}^{T} \sum_{i=1}^{N} \sum_{j=1}^{N}\left(e_{i s} e_{j s}\right)\left(w_{i t} w_{j t}\right)\right] \\
& \leq r\left(N^{2} T\right)^{-1} \sum_{s=1}^{T} \sum_{i=1}^{N} \sum_{j=1}^{N}\left[\mathbb{E}\left(e_{i s}^{2}\right) \mathbb{E}\left(e_{j s}^{2}\right)\right]^{1 / 2} \mathbb{E}\left[\left(\lambda_{i t}^{\prime} \mathbf{F}_{t}\right)\left(\lambda_{j t}^{\prime} \mathbf{F}_{t}\right)\right] \\
& \leq r^{3}\left(N^{2} T\right)^{-1} Q T \sup _{p, q} \sum_{i=1}^{N} \sum_{j=1}^{N} \mid \mathbb{E}\left[\lambda_{i p, t} \lambda_{j q, t} F_{p t} F_{q t} \mid\right. \\
& \leq r^{3}\left(N^{2} T\right)^{-1} Q T K_{1 N T}=r^{3} N^{-2} Q K_{1 N T}
\end{aligned}
$$

Novamente somando sobre $t$ e dividendo por $T$, obtemos

$$
T^{-1} \sum_{t=1}^{T}\left\|D_{7 t}\right\|^{2}=O_{p}\left(N^{-2} K_{1 N T}\right) .
$$

Por fim, para $D_{8 t}$ 


$$
\begin{aligned}
\left\|D_{8 t}\right\|^{2} & =(N T)^{-2}\left\|\tilde{\mathbf{F}}^{\prime} w e_{t}\right\|^{2} \\
& =\left(N^{2} T\right)^{-1} \frac{\left\|\tilde{\mathbf{F}}^{\prime}\right\|^{2}}{T}\left\|w e_{t}\right\|^{2} \\
\mathbb{E}\left[\left\|D_{8 t}\right\|^{2}\right] & \leq r\left(N^{2} T\right)^{-1} \sum_{s=1}^{T} \sum_{i=1}^{N} \sum_{j=1}^{N} \mathbb{E}\left[w_{i s} w_{j s} e_{i t} e_{j t}\right]
\end{aligned}
$$

Por um procedimento similar ao termo $D_{7 t}$, obtemos

$$
T^{-1} \sum_{t=1}^{T}\left\|D_{8 t}\right\|^{2}=O_{p}\left(N^{-2} K_{1 N T}\right)
$$

Concluímos que o lado direito da equação (A.12) é soma de variáveis de ordens

$$
\left\{\frac{1}{N}, \frac{1}{N T}, \frac{K_{1 N T}}{N^{2}}, \frac{K_{2 N T}}{N^{2} T^{2}}, \frac{K_{3 N T}}{N^{2} T^{2}}\right\},
$$

portanto segue-se o teorema.

Prova Proposição 3.1.1. Notemos que podemos reescrever

$$
\frac{\tilde{\mathbf{F}}^{\prime} \mathbf{F}}{T}=\frac{(\tilde{\mathbf{F}}-\mathbf{F} H)^{\prime} \mathbf{F}}{T}+\frac{H^{\prime} \mathbf{F}^{\prime} \mathbf{F}}{T}=\frac{H^{\prime} \mathbf{F}^{\prime} \mathbf{F}}{T}+O_{p}\left(C_{N T}^{-2}\right),
$$

uma vez que $(\tilde{\mathbf{F}}-\mathbf{F} H)^{\prime} \mathbf{F} / T=O_{p}\left(C_{N T}^{-2}\right)$, ver lema B.2 de Bai (2003). Agora multiplicando por $H$ à direita obtemos

$$
\frac{\tilde{\mathbf{F}}^{\prime} \mathbf{F} H}{T}=\frac{H^{\prime} \mathbf{F}^{\prime} \mathbf{F} H}{T}+O_{p}\left(C_{N T}^{-2}\right) .
$$

Reescrevendo o lado esquerdo temos

$$
\begin{aligned}
\frac{\tilde{\mathbf{F}}^{\prime} \mathbf{F} H}{T} & =\frac{\tilde{\mathbf{F}}^{\prime}(\mathbf{F} H-\tilde{\mathbf{F}}+\tilde{\mathbf{F}})}{T} \\
& =\frac{\tilde{\mathbf{F}}^{\prime}(\mathbf{F} H-\tilde{\mathbf{F}})}{T}+\frac{\tilde{\mathbf{F}}^{\prime} \tilde{\mathbf{F}}}{T} \\
& =O_{p}\left(C_{N T}^{-2}\right)+\mathbb{I}_{r},
\end{aligned}
$$

já que $\tilde{\mathbf{F}}^{\prime}(\mathbf{F} H-\tilde{\mathbf{F}}) / T=O_{p}\left(C_{N T}^{-2}\right)$ e $\tilde{\mathbf{F}}^{\prime} \tilde{\mathbf{F}} / T=\mathbb{I}_{r}$, ver lema B.3 de Bai (2003). Relacionando as equações (A.14) e (A.15) obtemos

$$
\mathbb{I}_{r}=\frac{H^{\prime} \mathbf{F}^{\prime} \mathbf{F} H}{T}+O_{p}\left(C_{N T}^{-2}\right) .
$$

Assim, se $\mathbf{F}^{\prime} \mathbf{F} / T=\mathbb{I}_{r}$, temos $\mathbb{I}_{r}=H^{\prime} H+O_{p}\left(C_{N T}^{-2}\right)$. Para $N, T \rightarrow \infty$, podemos assumir que $H$ é uma matriz ortogonal com autovalores $1 \mathrm{ou}-1$.

Da definição da matriz de rotação $H$ obtemos que 


$$
H^{\prime}=V_{N T}^{-1}\left(\tilde{\mathbf{F}}^{\prime} \mathbf{F} / T\right)\left(\boldsymbol{\Lambda}_{0}^{\prime} \boldsymbol{\Lambda}_{0} / N\right)=V_{N T}^{-1} H^{\prime}\left(\boldsymbol{\Lambda}_{0}^{\prime} \boldsymbol{\Lambda}_{0} / N\right)+O_{p}\left(C_{N T}^{-2}\right)
$$

isso é empregando o fato $\left(\tilde{\mathbf{F}}^{\prime} \mathbf{F} / T\right)=H^{\prime}+O_{p}\left(C_{N T}^{-2}\right) \operatorname{sob} \mathbf{F}^{\prime} \mathbf{F} / T=\mathbb{I}_{r}$ na relação (A.13). Multiplicando por $V_{N T}$ em ambos os lados e aplicando transposta temos

$$
\left(\boldsymbol{\Lambda}_{0}^{\prime} \boldsymbol{\Lambda}_{0} / N\right) H=H V_{N T}+O_{p}\left(C_{N T}^{-2}\right)
$$

Esta equação implica que $H$ (salvo um termo desprezável) é uma matriz cujas colunas são os autovetores de $\left(\boldsymbol{\Lambda}_{0}^{\prime} \boldsymbol{\Lambda}_{0} / N\right)$. A última matriz é diagonal com autovalores distintos por hipóteses, portanto, cada autovalor é associado com um único autovetor unitário (salvo um signo) e cada autovetor tem um elemento não zero. Isso implica que $H$ é uma matriz diagonal salvo um termo $O_{p}\left(C_{N T}^{-2}\right)$, o qual implica que $H=\mathbb{I}_{r}+O_{p}\left(C_{N T}^{-2}\right)$.

Lema A.2.2. Considerando que as suposições (S6.a-S6.e) são satisfeitas, temos

$$
\left(\frac{1}{\sqrt{T}}\right) G\left(P^{\prime} e\right) \stackrel{d}{\rightarrow} Z
$$

onde $Z$ é uma variável aleatória com distribuição normal multivariada com média nula e matriz de covariâncias $\Gamma^{*-1}=\lim _{T \rightarrow \infty} \frac{G \mathbb{E}\left[P^{\prime} P\right] G^{\prime}}{T}$ e G é uma matriz com mesmo número de colunas de P.

Prova. Seja $p_{j}$ a entrada da $j$-ésima linha de $P^{\prime} e$, o qual tem a forma

$$
p_{j}=\sum_{t=1}^{T} p_{j t}=\sum_{t=1}^{T} h_{j t} F_{j t} e_{t}
$$

em que $\left\{e_{t}\right\}_{t=1}^{N}$ são processos gaussianos independentes, $F_{j t}$ é o processo fatorial estacionário e $h_{j t}$ é uma função produto das ondaletas e uma função devido ao processo de ortonormalização. Notemos que $P^{\prime} e$ é um vetor composto por elementos $p_{j}$, e portanto pode ser escrito como $P^{\prime} e=\sum_{t=1}^{T} \mathbf{q}_{t^{\prime}}$ em que $\mathbf{q}_{t}$ é um vetor cujos elementos são $p_{j t}$. Além disso temos que

$$
\mathbb{E}\left[\mathbf{q}_{t} \mid \mathcal{F}_{t-1}\right]=0
$$

e como consequência $\left\{\mathbf{q}_{t}\right\}_{t=1}^{\infty}$ é uma sequência de vetores diferenças martingais. Temos também que

$$
\begin{aligned}
\mathbb{E}\left(\frac{\sum_{t=1}^{T} \mathbf{q}_{t} \mathbf{q}_{t}^{\prime}}{T}\right) & =\mathbb{E}\left(\frac{P^{\prime} e e^{\prime} P}{T}\right) \\
& =\mathbb{E}\left[\mathbb{E}\left(\frac{P^{\prime} e e^{\prime} P}{T} \mid P\right)\right] \\
& =\mathbb{E}\left(\frac{P^{\prime} P}{T}\right)
\end{aligned}
$$

Seja $G$ uma matriz com mesmo número de colunas de $P$, portanto temos do Lema 3.4.1 


$$
\Gamma^{*-1}=\lim _{T \rightarrow \infty} \frac{G \mathbb{E}\left[P^{\prime} P\right]^{-1} G^{\prime}}{T}
$$

Portanto, empregando o teorema do limite central para sequência de vetores diferenças martingais, obtemos o resultado.

Prova Teorema 3.4.1. Definamos as seguintes quantidades: seja $P=\Sigma_{w}^{-1 / 2} \boldsymbol{\Theta}$ e $\mathbf{X}=\Sigma_{w}^{-1 / 2} \operatorname{vec}(\mathbf{Y})$. Portanto pela definição do estimador para os coeficientes de ondaletas temos

$$
\begin{aligned}
\hat{\boldsymbol{\beta}} & =\left[P^{\prime} P\right]^{-1} P^{\prime} \mathbf{X} \\
& =\left[P^{\prime} P\right]^{-1} P^{\prime}\left(\Sigma_{w}^{-1 / 2} \operatorname{vec}(\mathbf{Y})\right) \\
& =\left[P^{\prime} P\right]^{-1} P^{\prime}\left[\Sigma_{w}^{-1 / 2}(\boldsymbol{\Theta} \boldsymbol{\beta}+\operatorname{vec}(\mathbf{w}))\right] \\
& =\left[P^{\prime} P\right]^{-1} P^{\prime}\left[\Sigma_{w}^{-1 / 2} \boldsymbol{\Theta} \boldsymbol{\beta}+\Sigma_{w}^{-1 / 2} \operatorname{vec}(\mathbf{w})\right] \\
& =\left[P^{\prime} P\right]^{-1} P^{\prime}\left[P \boldsymbol{\beta}+\Sigma_{w}^{-1 / 2}(\mathbf{R}+\epsilon)\right], \quad \operatorname{vec}(\mathbf{w})=\mathbf{R}+\epsilon \\
& =\left[P^{\prime} P\right]^{-1} P^{\prime}\left[P \boldsymbol{\beta}+\Sigma_{w}^{-1 / 2} \mathbf{R}+\Sigma_{w}^{-1 / 2} \epsilon\right] \\
& =\left[P^{\prime} P\right]^{-1} P^{\prime} P \boldsymbol{\beta}+\left[P^{\prime} P\right]^{-1} P^{\prime} \Sigma_{w}^{-1 / 2} \mathbf{R}+\left[P^{\prime} P\right]^{-1} P^{\prime} \Sigma_{w}^{-1 / 2} \epsilon \\
& =\boldsymbol{\beta}+\left[P^{\prime} P\right]^{-1} P^{\prime} \Sigma_{w}^{-1 / 2} \mathbf{R}+\left[P^{\prime} P\right]^{-1} P^{\prime} \Sigma_{w}^{-1 / 2} \epsilon \\
& =\boldsymbol{\beta}+A+B,
\end{aligned}
$$

em que $A=\left[P^{\prime} P\right]^{-1} P^{\prime} \Sigma_{w}^{-1 / 2} \mathbf{R}$ e $B=\left[P^{\prime} P\right]^{-1} P^{\prime} \Sigma_{w}^{-1 / 2} \epsilon$. Note que $\mathbf{R}=\operatorname{vec}\left(R_{F}(t, J)\right)$ e $\epsilon=\operatorname{vec}(\mathbf{e})$ onde $R_{F}^{i}(t, J)=w_{i t}-e_{i t} \operatorname{com} i=1, \ldots, N$ em que $R_{F}^{i}(t, J)$ é um processo dependente dos fatores e dos erros devido ao truncamento da expansão de ondaletas da $i$ - ésima linha da matriz de cargas fatoriais, o qual foi definido no Teorema 3.3.1. Da propriedade (3.29) temos que para os coeficientes de ondaletas

$$
\sup \left(\sum_{j \geq J} \sum_{k}\left\|\beta_{j k}\right\|^{2}\right)=O\left(T^{-\frac{2 m}{2 m+1}}\right) .
$$

Portanto

$$
\begin{aligned}
\|A\|^{2} & =\left\|\left[P^{\prime} P\right]^{-1}\right\|^{2} \cdot\left\|P^{\prime} \Sigma_{w}^{-1 / 2} \mathbf{R}\right\|^{2} \\
& =O_{p}\left[\left(2^{-J \cdot s}+T^{-1 / 2} 2^{-J \cdot(l-1 / 2-1 /(2 \cdot p))}\right) \sqrt{\log T}\right] \\
& =O_{p}\left(T^{-1 / 2-\tau}\right),
\end{aligned}
$$

para algum $\tau>0$. Os parâmetros de suavidade e forma $m, s, l$ e $p$, das cargas fatoriais, são definidos na suposição (S6) da Seção 3.4. Assim $\|A\|^{2}=O_{p}\left(T^{-1 / 2} \cdot T^{-\tau}\right)$. Como consequência $\sqrt{T}\|A\|^{2}=O_{p}\left(T^{-\tau}\right)=$ 
$o_{p}(1)$. Para derivar a distribuição assintótica de $\boldsymbol{\beta}$, do Lema 3.4.1 temos

$$
T H_{T}\left(P^{\prime} P\right)^{-1} H_{T}^{\prime} \stackrel{p}{\rightarrow} \Gamma
$$

em que $\Gamma=\lim _{T \rightarrow \infty} T H_{T} \mathbb{E}\left(P^{\prime} P\right)^{-1} H_{T}^{\prime}$. Do Lema A.2.2 e do Teorema de Slutsky obtemos

$$
\sqrt{T} H_{T}(\hat{\boldsymbol{\beta}}-\boldsymbol{\beta})=\sqrt{T} H_{T} A+\sqrt{T} H_{T} B=o_{p}(1)+\mathbf{Z},
$$

em que $\mathbf{Z}$ é um vetor aleatório com distribuição normal $k$-dimensional com média nula e matriz de covariância $\Gamma$. 